\title{
MACROSCOPIC LIMIT OF A ONE-DIMENSIONAL MODEL FOR AGING FLUIDS
}

\author{
DAVID BENOIT ${ }^{\dagger}$, CLAUDE LE BRIS $^{\dagger}$, , AND TONY LELIÈVRE ${ }^{\dagger}$
}

\begin{abstract}
We study a one-dimensional equation arising in the multiscale modeling of some non-Newtonian fluids. At a given shear rate, the equation provides the instantaneous mesoscopic response of the fluid, allowing to compute the corresponding stress. In a simple setting, we study the well-posedness of the equation and next the long-time behavior of its solution. In the limit of a response of the fluid much faster than the time variations of the ambient shear rate, we derive some equivalent macroscopic differential equations that relate the shear rate and the stress. Our analytical conclusions are confronted to some numerical experiments. The latter quantitatively confirm our derivations.
\end{abstract}

Key words. Non-Newtonian fluids; micro-macro model; longtime behavior.

AMS subject classifications. 35Q35,76A05,35B40

\section{Introduction.}

Generalities. The present contribution is devoted to the mathematical analysis of the equation

$$
\frac{\partial p}{\partial t}(t, \sigma)+\dot{\gamma}(t) \frac{\partial p}{\partial \sigma}(t, \sigma)=-\chi(\sigma) p(t, \sigma)+\left(\int \chi(\sigma) p(t, \sigma) d \sigma\right) \delta_{0}(\sigma) .
$$

Equation (1.1) arises in the modeling of some non-Newtonian fluid flows. Some details on the modeling will be given below. The variable $\sigma$ is one-dimensional, varies on the real line $\mathbb{R}$, and models a quantity homogeneous to a stress (actually to a certain entry of the stress tensor). The variable $t$ of course denotes the time, and equation (1.1) is supplied with some initial condition $p(t=0,)=.p_{0}($.$) . The unknown real-valued$ function $p(t, \sigma)$, solution to 1.1 , satisfies the two properties: it is nonnegative

$$
p(t, \sigma) \geq 0, \quad \text { for all } t \geq 0 \text { and } \sigma \in \mathbb{R},
$$

and normalized to one

$$
\int_{-\infty}^{+\infty} p(t, \sigma) d \sigma=1
$$

for all times $t \geq 0$. The function $p$ models the density of probability to have a certain (elementary microscopic) stress $\sigma$, at time $t$, at a macroscopic space position $x$. The actual, deterministic stress within the fluid is thus given by $\tau=\int \sigma p(t, \sigma) d \sigma$, of Equation 1.5 below. Equation 1.1 is thus implicitly parameterized by this position $x$ (thus the multiscale nature of the problem, as will be seen below). The function $\dot{\gamma}$, also a function of time, is assumed given. It models the shear rate at the position $x$, under which we wish to compute, using (1.1), the mesoscopic response of the fluid. The notation $\dot{\gamma}$ is traditional in Fluid Mechanics, hence its use here. In (1.1), we denote by $\chi$ the characteristic function

$$
\chi=\mathbb{1}_{\mathbb{R} \backslash\left[-\sigma_{c}, \sigma_{c}\right]}
$$

\footnotetext{
${ }^{\dagger}$ Ecole des Ponts and INRIA, 6 \& 8 Av. B. Pascal, 77455 Marne-la-Vallée, France. ( $\{$ benoitd,lebris,lelievre $\} @$ cermics.enpc.fr)

${ }^{\ddagger}$ Corresponding author
} 
where $\sigma_{c}>0$ a scalar positive parameter, fixed once and for all. It models some local threshold value of the stress, which plays a crucial role in the modeling. As is usual, we denote by $\delta_{0}$ the Dirac mass at zero. From the definitions of $\chi$ and $\delta_{0}$, it is immediately seen that, at least formally (and this will indeed be made rigorous, see Lemmata 4.1 and 4.2 in Section 4 below), equation (1.1) preserves in time the two properties $(1.2)$ and $(1.3)$.

Two quantities are typically computed using the solution $p$ to (1.1): first the so-called fluidity

$$
f(t)=\int \chi(\sigma) p(t, \sigma) d \sigma
$$

and next the (real-valued) stress

$$
\tau(t)=\int \sigma p(t, \sigma) d \sigma
$$

Our purpose in this article is to mathematically study equation (1.1) (in terms of existence and uniqueness of the solution $p$, properties and long-time behavior of that solution) and to derive a macroscopic equation equivalent to this equation. By macroscopic equation, we mean an equation (actually a differential equation, or a system of differential equations) that directly relates the shear rate $\dot{\gamma}$, the fluidity $f$ and the stress $\tau$ without the explicit need to compute $p$. In these macroscopic equations, the scalar $f(t)$ will be the inverse of the mechanical relaxation time, thus its name "fluidity". We will be able, in particular, to obtain a macroscopic equation which is close to models that have been proposed for aging fluids [7, 12, see the discussion at the end of Section 7 .

Some elements on the modeling. Equation (1.1) is the simplest possible form of an equation describing the mesoscopic behavior of a complex fluid, such as a concentrated suspension, or more generically a soft amorphous material, with properties intermediate between those of a fluid and those of a solid. These materials exhibit a highly non-Newtonian behavior and may give rise to a macroscopic yield stress.

At low stress, such a material behaves in an elastic way. But above a certain stress threshold, here denoted by the critical value $\sigma_{c}$, one observes a relaxation toward a completely relaxed state. This behavior is modeled by equation (1.1). The probability of finding the fluid in the state of stress $\sigma$ at time $t$ evolves in time for two different reasons: the term $\dot{\gamma}(t) \frac{\partial p}{\partial \sigma}$ models the modification of the stress induced by the existence of the shear rate, while the term $-\chi p+\left(\int \chi p\right) \delta_{0}$ encodes the relaxation toward zero of the part of the stress above the threshold $\sigma_{c}$. From a probabilistic viewpoint, the stochastic process $\left(\Sigma_{t}\right)_{t \geq 0}$ associated to the Fokker-Planck equation 1.1 evolves deterministically when $\left|\Sigma_{t}\right| \leq \sigma_{c}$ and jumps to zero with an exponential rate 1 when $\left|\Sigma_{t}\right| \geq \sigma_{c}$. The process $\left(\Sigma_{t}\right)_{t \geq 0}$ belongs to the class of piecewise-deterministic Markov processes, which have been introduced in the probabilistic literature in the 1980's for biological modeling for example. In particular, coupling arguments have been proposed to study the longtime behavior of such processes (see [1]). We argue on the Fokker-Planck equation and proceed differently. The argument we are using here to study the longtime behavior is purely deterministic in nature, and is based on a delay equation related to the Fokker-Planck equation (1.1). 
We would like to mention that for more realistic models, a third phenomenon is typically at play, in addition to the stress induced by the ambient fluid, and to the relaxation to zero. All states of stress are not independent of one another, and they may also depend on the state of stress at neighboring points within the fluid. A certain redistribution of the stress therefore always occurs. This redistribution can be encoded in various ways, depending on some more detailed elements of modeling. In the so-called Hébraud-Lequeux model introduced in the seminal article [11] (and then extensively studied mathematically in the works [5, 6, 8), the redistribution is performed by a diffusion term in the stress space, at the given location $x$ in the ambient physical space, and the complete equation thus writes

$$
\frac{\partial p}{\partial t}+\dot{\gamma}(t) \frac{\partial p}{\partial \sigma}=-\chi p+\left(\int \chi p\right) \delta_{0}(\sigma)+\alpha\left(\int \chi p\right) \frac{\partial^{2} p}{\partial \sigma^{2}}
$$

where $\alpha>0$ is some parameter. In an alternative model introduced by Bocquet and coll. in [4, the redistribution is achieved by some type of local "convolution" in the physical space. The equation (we recall, set at the physical location $x$ ) writes

$$
\begin{aligned}
& \frac{\partial p}{\partial t}(t, x, \sigma)+\dot{\gamma}(t, x) \frac{\partial p}{\partial \sigma}(t, x, \sigma)=-\chi(\sigma) p(t, x, \sigma)+\left(\int d \sigma^{\prime} \chi\left(\sigma^{\prime}\right) p\left(t, x, \sigma^{\prime}\right)\right) \delta_{0}(\sigma) . \\
& \quad+\iint d x^{\prime} d \sigma^{\prime} \chi\left(\sigma^{\prime}\right)\left(p\left(t, x^{\prime}, \sigma^{\prime}\right) p\left(t, x, \sigma-G\left(x, x^{\prime}\right) \sigma^{\prime}\right)-p\left(t, x^{\prime}, \sigma^{\prime}\right) p(t, x, \sigma)\right) .
\end{aligned}
$$

with a function $G\left(x, x^{\prime}\right)$ related to the Green function of some local Stokes-type problem.

The equation 1.1 which we study in the present article ignores the redistribution phenomenon, which amounts to taking $\alpha=0$ in (1.6) or $G \equiv 0$ in (1.7). In the absence of such a simplification, we are unable to proceed with the main result of this article, which is the derivation of the macroscopic equation from our multiscale model. The well posedness result contained in our Theorem 3.1. on the other hand, also holds for (1.6) whith $\alpha>0$ and has indeed been established some years ago in [5]. Some more detailed comments on the modeling, as well as some formal foundations of the model based on a system of interacting particles are presented in [3].

Plan of our contribution. Our article is organized as follows. To start with, we study in Section 2 the stationary solutions to 1.1). We next show in Section 3 existence and uniqueness of the solutions to the time-dependent equation (1.1). Our result is stated in Theorem 3.1. Section 4 follows, establishing some useful properties of the solution. In order to understand the macroscopic equivalent of equation 1.1 for a given shear rate $\dot{\gamma}(t)$, which we assume varies slowly as compared to the characteristic time of equation (1.1), we need to understand the long-time behavior of the solution to (1.1). We therefore study this behavior in Sections 5 and 6 , respectively in the case of a constant shear rate $\dot{\gamma}(t) \equiv \dot{\gamma}_{\infty}$, and in the case of a slowly varying shear rate $\dot{\gamma}(\epsilon t)$. The results are stated in Theorems 5.1 and 6.1. We are then in position to derive, in Section 7, the macroscopic differential equations equivalent to (1.1) in this limit, namely system (7.8). Our final section, Section 8, presents some numerical experiments which confirm and illustrate our theoretical results.

2. Stationary states. We study in this section the stationary states of (1.1). We therefore assume that $\dot{\gamma}(t) \equiv \dot{\gamma}_{\infty}$ is a fixed scalar and consider the solutions 
$p_{\infty}: \mathbb{R} \rightarrow \mathbb{R}$ to the following equation

$$
\dot{\gamma}_{\infty} \frac{d p_{\infty}}{d \sigma}=-\chi p_{\infty}+\left(\int \chi p_{\infty}\right) \delta_{0} \text { in } \mathcal{D}^{\prime}(\mathbb{R}) .
$$

Here and in the following, for a subset $I \subset \mathbb{R}^{d}, \mathcal{D}^{\prime}(I)$ denotes the set of distributions on $I$. By convention, since the time-dependent version of the equation is linear and formally preserves positiveness and the integral over the real line, we are only interested in the stationary solutions $p_{\infty}$ that additionally satisfy 1.2 and $[1.3$, that is,

$$
p_{\infty} \in L^{1}(\mathbb{R}), \quad p_{\infty} \geq 0, \quad \text { a.e. and } \quad \int p_{\infty}=1
$$

We have the following result:

Lemma 2.1. When $\dot{\gamma}_{\infty}=0$, the solutions to 2.1)-2.2 are exactly all nonnegative normalized densities with compact support in $\left[-\sigma_{c}, \sigma_{c}\right]$. When $\dot{\gamma}_{\infty} \neq 0$, there exists a unique solution $p_{\infty}$ to 2.1)-2.2.

Proof. In the case $\dot{\gamma}_{\infty}=0$, the equation 2.1) implies $p_{\infty}=0$ in $\mathcal{D}^{\prime}\left(\mathbb{R} \backslash\left[-\sigma_{c}, \sigma_{c}\right]\right)$, hence the result. Up to a change of $p_{\infty}$ into $\sigma \rightarrow p_{\infty}(-\sigma)$, we may, without loss of generality, consider only the case $\dot{\gamma}_{\infty}>0$ for our proof. We first note that $p_{\infty}$ defined by

$$
p_{\infty}(\sigma)= \begin{cases}0 & \text { if } \sigma<0 \\ \frac{1}{\sigma_{c}+\dot{\gamma}_{\infty}} & \text { if } 0<\sigma \leq \sigma_{c} \\ \frac{1}{\sigma_{c}+\dot{\gamma}_{\infty}} \mathrm{e}^{-\left(\sigma-\sigma_{c}\right) / \dot{\gamma}_{\infty}} & \text { if } \sigma_{c}<\sigma .\end{cases}
$$

is a solution to 2.1, hence the existence result.

We now show uniqueness. By linearity, we assume that $p_{\infty} \in L^{1}(\mathbb{R})$ is a solution of 2.1] with $\int_{\mathbb{R}} p_{\infty}=0$ and show that $p_{\infty}=0$. Equation 2.1 implies $\dot{\gamma}_{\infty} \frac{d p_{\infty}}{d \sigma}+p_{\infty}=$ 0 in $\mathcal{D}^{\prime}\left(\mathbb{R} \backslash\left[-\sigma_{c}, \sigma_{c}\right]\right)$. Because $p_{\infty} \in L^{1}(\mathbb{R})$, this leads to $p_{\infty}=\alpha \mathrm{e}^{-\frac{\sigma}{\gamma_{\infty}}}$ a.e. on $\left(\sigma_{c}, \infty\right)$ with $\alpha$ a scalar and $p_{\infty}=0$ a.e. on $\left(-\infty,-\sigma_{c}\right)$.

In the case $\alpha=0$, this implies $\chi p_{\infty}=0$. Consequently, 2.1) rewrites $\frac{d p_{\infty}}{d \sigma}=0$ on the whole real line. This readily implies $p_{\infty}=0$ since $\int_{\mathbb{R}} p_{\infty}=0$.

In the case $\alpha \neq 0$, we obtain that 2.1) writes

$$
\dot{\gamma}_{\infty} \frac{d p_{\infty}}{d \sigma}=\alpha\left(\int_{\sigma_{c}}^{\infty} \mathrm{e}^{-\frac{\sigma^{\prime}}{\gamma}} d \sigma^{\prime}\right) \delta_{0} \text { in } \mathcal{D}^{\prime}\left(-\infty, \sigma_{c}\right)
$$

so that $p_{\infty}=\frac{\alpha}{\dot{\gamma}_{\infty}}\left(\int_{\sigma_{c}}^{\infty} \mathrm{e}^{-\frac{\sigma^{\prime}}{\dot{\gamma}_{\infty}}} d \sigma^{\prime}\right) \mathbb{1}_{\mathbb{R}_{+}}(\sigma)$ a.e. on $\left(-\infty, \sigma_{c}\right)$. Using that $p_{\infty}=\alpha \mathrm{e}^{-\frac{\sigma}{\dot{\gamma} \infty}}$ a.e. on $\left(\sigma_{c}, \infty\right)$ and $\int p_{\infty}=0$, we find $\alpha=0$ and thus $p_{\infty}=0$. This concludes the proof.

3. Existence and uniqueness. This section is devoted to the proof of the following result:

ThEOREm 3.1 (Existence and uniqueness). Consider $\dot{\gamma}$ a function of time that satisfies

$$
\dot{\gamma} \in L_{\mathrm{loc}}^{1}(0, \infty) \text { and } \dot{\gamma} \geq m_{\dot{\gamma}} \text { a.e. where } m_{\dot{\gamma}}>0 \text { is a fixed scalar. }
$$


Denote by $\gamma(t)=\int_{0}^{t} \dot{\gamma}(s) d s$. Consider $p_{0} \in L^{1}(\mathbb{R})$. Then, for any $T>0$, there exists a unique $p$ in $C^{0}\left([0, T) ; L^{1}\right)$ such that $p(0, \sigma)=p_{0}(\sigma)$ for almost all $\sigma \in \mathbb{R}$ and such that 1.1) holds for $p$ in the sense of distributions on $(0, T) \times \mathbb{R}$. In addition, introduce

$$
A(t)=\int \chi(\sigma) p_{0}(\sigma-\gamma(t)) \mathrm{e}^{-\int_{0}^{t} \chi(\sigma-\gamma(t)+\gamma(u)) d u} d \sigma
$$

and $\phi$ defined by induction on $k \in \mathbb{N}^{*}$ as follows

$$
\phi(t)=A(t)
$$

$$
+ \begin{cases}0 & \text { when } t \in\left(0, \gamma^{-1}\left(\sigma_{c}\right)\right) \\ \int_{0}^{\gamma^{-1}\left(\gamma(t)-\sigma_{c}\right)} \phi(s) \mathrm{e}^{-t+\gamma^{-1}\left(\gamma(s)+\sigma_{c}\right)} d s & \text { when } t \in\left(\gamma^{-1}\left(k \sigma_{c}\right), \gamma^{-1}\left((k+1) \sigma_{c}\right)\right) .\end{cases}
$$

Both $A$ and $\phi$ belong to $L^{\infty}(0, T)$. Then, the solution $p$ to $(1.1)$ is explicitly given by

$$
\begin{aligned}
p(t, \sigma)= & p_{0}(\sigma-\gamma(t)) \mathrm{e}^{-\int_{0}^{t} \chi(\sigma-\gamma(t)+\gamma(u)) d u} \\
& +\frac{\phi \circ \gamma^{-1}(\gamma(t)-\sigma)}{\dot{\gamma} \circ \gamma^{-1}(\gamma(t)-\sigma)} \mathrm{e}^{-\int_{\gamma^{-1}(\gamma(t)-\sigma)}^{t} \chi(\sigma-\gamma(t)+\gamma(u)) d u} \mathbb{1}_{(0, \gamma(t))}(\sigma)
\end{aligned}
$$

and $f$ defined by 1.4 is equal to $\phi$ :

$$
f=\phi \text { a.e. on }(0, T) \text {. }
$$

REMARK 1. The above results also hold for $\dot{\gamma}<-m_{\dot{\gamma}}$ negative. However, it is unclear how to extend these results if $\dot{\gamma}$ is allowed to vanish.

REMARK 2. We will see in the next section that if the initial condition $p_{0}$ is nonnegative and normalized, then this property is preserved in time for the solution $p$.

Proof. We first note that

$$
\gamma(t)=\int_{0}^{t} \dot{\gamma}(s) d s
$$

is a strictly increasing continuous function of the time because of 3.1). Throughout this proof, we assume $T>\gamma^{-1}\left(\sigma_{c}\right)$. When $T \leq \gamma^{-1}\left(\sigma_{c}\right)$, the arguments are similar and actually simpler. We first show uniqueness, then $p$ given by (3.4) belongs to $C^{0}\left([0, T) ; L^{1}\right)$ and is a solution of 1.1$)$ in $\mathcal{D}^{\prime}((0, T) \times \mathbb{R})$.

Step 1: Uniqueness. Equation (1.1) is linear, we therefore consider a solution $p \in C^{0}\left([0, T) ; L^{1}\right)$ associated to the zero initial condition $p_{0}=0$ and we intend to show that $p=0$. Denote by

$$
\tilde{p}(t, \xi)=p(t, \xi+\gamma(t)) \mathrm{e}^{\int_{0}^{t} \chi(\xi+\gamma(u)) d u} .
$$

We now show

$$
\frac{\partial \tilde{p}}{\partial t}(t, \xi)=f(t) \delta_{-\gamma(t)}(\xi) \mathrm{e}^{\int_{0}^{t} \chi(-\gamma(t)+\gamma(u)) d u} \quad \text { in } \mathcal{D}^{\prime}((0, T) \times \mathbb{R})
$$


with $f$ defined from $p$ by (1.4). We have, for all $\psi \in \mathcal{D}((0, T) \times \mathbb{R})$, (where $\mathcal{D}((0, T) \times \mathbb{R})$ denotes the set of $C^{\infty}$ functions with compact support in $\left.(0, T) \times \mathbb{R}\right)$

$$
\begin{aligned}
-\int_{0}^{T} \int_{\mathbb{R}} \tilde{p} \frac{\partial \psi}{\partial t} & =-\int_{0}^{T} \int_{\mathbb{R}} p(t, \xi+\gamma(t)) \mathrm{e}^{\int_{0}^{t} \chi(\xi+\gamma(u)) d u} \frac{\partial \psi}{\partial t}(t, \xi) d \xi d t \\
& =-\int_{0}^{T} \int_{\mathbb{R}} p(t, \sigma) \frac{\partial \psi}{\partial t}(t, \sigma-\gamma(t)) \mathrm{e}^{\int_{0}^{t} \chi(\sigma-\gamma(t)+\gamma(u)) d u} d \sigma d t .
\end{aligned}
$$

For $n \in \mathbb{N}$, denote now by $\rho^{n}$ a mollifier, $\chi^{n}=\rho^{n} * \chi$ and

$$
\eta^{n}(t, \sigma)=\psi(t, \sigma-\gamma(t)) \mathrm{e}^{\int_{0}^{t} \chi^{n}(\sigma-\gamma(t)+\gamma(u)) d u} .
$$

The fact that $p$ is solution to 1.1$)$ in $\mathcal{D}^{\prime}((0, T) \times \mathbb{R})$ yields

$$
-\int_{0}^{T} \int_{\mathbb{R}} p\left(\frac{\partial \eta^{n}}{\partial t}+\dot{\gamma} \frac{\partial \eta^{n}}{\partial \sigma}-\chi \eta^{n}\right)=\int_{0}^{T} f(t) \eta^{n}(t, 0) d t .
$$

This rewrites

$$
\begin{array}{r}
-\int_{0}^{T} \int_{\mathbb{R}} p(t, \sigma) \frac{\partial \psi}{\partial t}(t, \sigma-\gamma(t)) \mathrm{e}^{\int_{0}^{t} \chi^{n}(\sigma-\gamma(t)+\gamma(u)) d u} d \sigma d t \\
+\int_{0}^{T} \int_{\mathbb{R}} p(t, \sigma) \eta^{n}(t, \sigma)\left(\chi-\chi^{n}\right)(\sigma) d \sigma d t \\
=\int_{0}^{T} f(t) \psi(t,-\gamma(t)) \mathrm{e}^{\int_{0}^{t} \chi^{n}(-\gamma(t)+\gamma(u)) d u} d t
\end{array}
$$

As $n$ goes to infinity, $\chi^{n}$ converges to $\chi$ in $L_{\text {loc }}^{1}(\mathbb{R})$ and for almost all $t \in(0, T), \sigma \in \mathbb{R}$,

$$
\int_{0}^{t} \chi^{n}(\sigma-\gamma(t)+\gamma(u)) d u \rightarrow \int_{0}^{t} \chi(\sigma-\gamma(t)+\gamma(u)) d u .
$$

Because $p$ and $\psi$ respectively belong to $L^{\infty}\left((0, T), L^{1}\right)$ and $\mathcal{D}((0, T) \times \mathbb{R})$, all terms of (3.8) are bounded from below and from above by an integrable function independent of $n$. We apply the dominated convergence theorem to pass to the limit in (3.8)

$$
\begin{array}{r}
-\int_{0}^{T} \int_{\mathbb{R}} p(t, \sigma) \frac{\partial \psi}{\partial t}(t, \sigma-\gamma(t)) \mathrm{e}^{\int_{0}^{t} \chi(\sigma-\gamma(t)+\gamma(u)) d u} d \sigma d t \\
=\int_{0}^{T} f(t) \psi(t,-\gamma(t)) \mathrm{e}^{\int_{0}^{t} \chi(-\gamma(t)+\gamma(u)) d u} d t
\end{array}
$$

hence (3.6), using (3.7).

Define $t^{*}=\gamma^{-1}\left(\sigma_{c}\right)$. We now show that $\tilde{p}=0$ in $L^{\infty}\left(0, t^{*} ; L^{1}\right)$, this will prove that $p=0$ in $L^{\infty}\left(0, t^{*} ; L^{1}\right)$.

From (3.6), we deduce that $\frac{\partial \tilde{p}}{\partial t}=0$ in $\mathcal{D}^{\prime}(\tilde{\Omega})$ with

$$
\tilde{\Omega}=\left(0, t^{*}\right) \times\left(\left(-\infty,-\gamma\left(t^{*}\right)\right) \cup(0, \infty)\right) .
$$

Using that $p_{0}=0$, we find $\tilde{p}=0$ in $\mathcal{D}^{\prime}(\tilde{\Omega})$ and therefore in $L^{\infty}\left(0, t^{*} ; L^{1}\left(\left(-\infty,-\gamma\left(t^{*}\right)\right) \cup\right.\right.$ $(0, \infty)))$. This implies $p=0$ in $L^{\infty}\left(0, t^{*} ; L^{1}\left(\left(-\infty,-\gamma\left(t^{*}\right)\right) \cup\left(\gamma\left(t^{*}\right), \infty\right)\right)\right)$. 
In particular, since $\gamma\left(t^{*}\right)=\sigma_{c}$, we have, for all $t \in\left(0, t^{*}\right), f(t)=\int \chi p(t, \cdot)=0$ and thus, the equation (3.6) reads $\frac{\partial \tilde{p}}{\partial t}=0$ in $\mathcal{D}^{\prime}\left(\left(0, t^{*}\right) \times \mathbb{R}\right)$. We deduce that $\tilde{p}=0$ and therefore that $p=0$ in $L^{\infty}\left(0, t^{*} ; L^{1}\right)$.

Taking $t^{*}$ as initial time, we find $p=0$ in $L^{\infty}\left(t^{*}, 2 t^{*} ; L^{1}\right)$ with the previous arguments. Iterating, we obtain $p=0$ in $L^{\infty}\left(0, T ; L^{1}\right)$. This concludes the proof of uniqueness.

Our next two steps are respectively devoted to proving that $p$ defined by (3.4) belongs to $C\left([0, T) ; L^{1}\right)$ and that it satisfies equation $[1.1)$.

Step 2: Regularity of expression (3.4). First, the function $A$ defined by (3.2) belongs to $L^{\infty}(0, T)$ (with $\|A\|_{L^{\infty}(0, T)} \leq\left\|p_{0}\right\|_{L^{1}}$ ) and therefore $\phi$ defined by the recurrence relation (3.3) also belongs to $L^{\infty}(0, T)$. This implies, for almost all $t \in$ $[0, T)$,

$$
\int|p(t, \sigma)| d \sigma \leq\left\|p_{0}\right\|_{L^{1}}+\frac{\gamma(T)}{m_{\dot{\gamma}}}\|\phi\|_{L^{\infty}(0, T)},
$$

that is $p$ defined by $(3.4)$ belongs to $L^{\infty}\left(0, T ; L^{1}\right)$. Denote

$$
\begin{aligned}
& p_{11}(t, \sigma)=p_{0}(\sigma-\gamma(t)) \\
& p_{12}(t, \sigma)=\int_{0}^{t} \chi(\sigma-\gamma(t)+\gamma(u)) d u \\
& p_{21}(t, \sigma)=\frac{\phi \circ \gamma^{-1}(\gamma(t)-\sigma)}{\dot{\gamma} \circ \gamma^{-1}(\gamma(t)-\sigma)} \mathbb{1}_{(0, \gamma(t))}(\sigma) \mathbb{1}_{\mathbb{R}_{+}^{*}}(t) \\
& p_{22}(t, \sigma)=\int_{\gamma^{-1}(\gamma(t)-\sigma)}^{t} \chi(\sigma-\gamma(t)+\gamma(u)) d u
\end{aligned}
$$

so that $p=p_{11} \mathrm{e}^{-p_{12}}+p_{21} \mathrm{e}^{-p_{22}}$. We now check that $p_{11}$ and $p_{21}$ belong to $C^{0}\left([0, T) ; L^{1}\right)$ and $p_{12}$ and $p_{22}$ belong to $C^{0}\left([0, T) ; L^{\infty}\right)$. Using that $x \mapsto \mathrm{e}^{-x}$ is 1-Lipschitz on $[0, \infty)$, this will prove that $p \in C^{0}\left([0, T) ; L^{1}\right)$.

Consider $\epsilon>0, t \in[0, T)$ and $h$ such that $t+h \in[0, T)$. By density of $\mathcal{D}(\mathbb{R})$ in $L^{1}(\mathbb{R})$, there exists $p_{\epsilon} \in \mathcal{D}(\mathbb{R})$ such that

$$
\left\|p_{\epsilon}-p_{0}\right\|_{L^{1}}<\epsilon
$$

We obtain

$$
\begin{aligned}
\left\|p_{11}(t+h, \cdot)-p_{11}(t, \cdot)\right\|_{L^{1}} & =\int_{\mathbb{R}}\left|p_{0}(\sigma-\gamma(t+h))-p_{0}(\sigma-\gamma(t))\right| d \sigma \\
& \leq 2\left\|p_{\epsilon}-p_{0}\right\|_{L^{1}}+\int_{\mathbb{R}}\left|p_{\epsilon}(\sigma-\gamma(t+h))-p_{\epsilon}(\sigma-\gamma(t))\right| d \sigma \\
& \leq 2 \epsilon+\int_{\mathbb{R}} g_{\epsilon}^{h}(\sigma) d \sigma
\end{aligned}
$$

with $g_{\epsilon}^{h}(\sigma)=\left|p_{\epsilon}(\sigma-\gamma(t+h))-p_{\epsilon}(\sigma-\gamma(t))\right|$. Moreover, by continuity of $p_{\epsilon}$ and $\gamma$, we have, for all $\sigma \in \mathbb{R}$,

$$
g_{\epsilon}^{h}(\sigma) \rightarrow 0 \text { as } h \rightarrow 0
$$


and $g_{\epsilon}^{h} \leq 2\left\|p_{\epsilon}\right\|_{L^{\infty}}$ on a bounded interval (depending on the support of $p_{\epsilon}$ and on $\gamma(T)$ ). Using Lebesgue dominated convergence Theorem, we deduce that there exists $\eta_{\epsilon}>0$ such that for all $h \in\left(-\eta_{\epsilon}, \eta_{\epsilon}\right)$,

$$
\int_{\mathbb{R}} g_{\epsilon}^{h}(\sigma) d \sigma \leq \epsilon
$$

so that

$$
\left\|p_{11}(t+h, \cdot)-p_{11}(t, \cdot)\right\|_{L^{1}} \leq 3 \epsilon
$$

This yields $p_{11} \in C^{0}\left([0, T) ; L^{1}\right)$.

We now turn to $p_{12}$. For almost all $\sigma \in \mathbb{R}$, we have

$$
\begin{aligned}
\left|p_{12}(t+h, \sigma)-p_{12}(t, \sigma)\right| \\
\leq \int_{0}^{t}|\chi(\sigma-\gamma(t+h)+\gamma(u))-\chi(\sigma-\gamma(t)+\gamma(u))| d u \\
\quad+\int_{t}^{t+h} \chi(\sigma-\gamma(t+h)+\gamma(u)) d u \\
\leq \frac{1}{m_{\dot{\gamma}}} \int_{\sigma}^{\sigma+\gamma(t)}|\chi(v-\gamma(t+h))-\chi(v-\gamma(t))| d v+h \\
\leq \frac{1}{m_{\dot{\gamma}}} \int_{\mathbb{R}}|\chi(v-\gamma(t+h))-\chi(v-\gamma(t))| d v+h .
\end{aligned}
$$

This leads to

$$
\left\|p_{12}(t+h, \cdot)-p_{12}(t, \cdot)\right\|_{L^{\infty}} \leq \frac{2}{m_{\dot{\gamma}}}(\gamma(t+h)-\gamma(t))+h
$$

which yields $p_{12} \in C^{0}\left([0, T) ; L^{\infty}\right)$.

For any $t \in(0, T)$ and $h$ such that $t+h \in[0, T)$, we have

$$
\begin{aligned}
\int_{\mathbb{R}}\left|p_{21}(t+h, \sigma)-p_{21}(t, \sigma)\right| d \sigma \\
\leq \int_{0}^{\gamma(t)}\left|\frac{\phi \circ \gamma^{-1}(\gamma(t+h)-\sigma)}{\dot{\gamma} \circ \gamma^{-1}(\gamma(t+h)-\sigma)}-\frac{\phi \circ \gamma^{-1}(\gamma(t)-\sigma)}{\dot{\gamma} \circ \gamma^{-1}(\gamma(t)-\sigma)}\right| d \sigma \\
\quad+\left|\int_{\gamma(t)}^{\gamma(t+h)} \frac{\phi \circ \gamma^{-1}(\gamma(t+h)-\sigma)}{\dot{\gamma} \circ \gamma^{-1}(\gamma(t+h)-\sigma)} d \sigma\right| .
\end{aligned}
$$

Since $\phi$ belongs to $L^{\infty}(0, T), \phi \circ \gamma^{-1}$ belongs to $L^{\infty}(0, \gamma(T)) \subset L^{1}(0, \gamma(T))$ and we introduce a sequence $\theta_{n}$ in $\mathcal{D}(0, \gamma(T))$ such that

$$
\theta_{n} \rightarrow \phi \circ \gamma^{-1} \text { in } L^{1}(0, \gamma(T))
$$


and obtain

$$
\begin{aligned}
\int_{\mathbb{R}}\left|p_{21}(t+h, \sigma)-p_{21}(t, \sigma)\right| d \sigma \\
\quad \leq \quad \frac{2}{m_{\dot{\gamma}}}\left\|\theta_{n}-\phi \circ \gamma^{-1}\right\|_{L^{1}(0, \gamma(T))} \\
\quad+\int_{0}^{\gamma(t)}\left|\frac{\theta_{n}(\gamma(t+h)-\sigma)}{\dot{\gamma} \circ \gamma^{-1}(\gamma(t+h)-\sigma)}-\frac{\theta_{n}(\gamma(t)-\sigma)}{\dot{\gamma} \circ \gamma^{-1}(\gamma(t)-\sigma)}\right| d \sigma \\
\quad+\frac{\gamma(t+h)-\gamma(t)}{m_{\dot{\gamma}}}\|\phi\|_{L^{\infty}(0, T)} .
\end{aligned}
$$

Using the dominated convergence theorem for the second term, this implies that $\int_{\mathbb{R}}\left|p_{21}(t+h, \sigma)-p_{21}(t, \sigma)\right| d \sigma$ vanishes with $h$. We have obtained that $p_{21}$ belongs to $C^{0}\left((0, T) ; L^{1}\right)$. The continuity holds also at $t=0$ because for all $h \in(0, T)$

$$
\begin{aligned}
\int_{\mathbb{R}}\left|p_{21}(h, \sigma)-0\right| d \sigma & \leq \int_{0}^{\gamma(h)}\left|\frac{\phi \circ \gamma^{-1}(\gamma(h)-\sigma)}{\dot{\gamma} \circ \gamma^{-1}(\gamma(h)-\sigma)}\right| d \sigma \\
& \leq \frac{\gamma(h)}{m_{\dot{\gamma}}}\|\phi\|_{L^{\infty}(0, T)} .
\end{aligned}
$$

Finally, the function $p_{3}=p_{12}-p_{22}$ is in $C^{0}\left((0, T) ; L^{\infty}\right)$ (and so is $\left.p_{22}\right)$. Indeed, for any $t \in[0, T)$ and $h$ such that $t+h \in[0, T)$, for almost all $\sigma \in \mathbb{R}$,

$$
\begin{aligned}
& \left|p_{3}(t+h, \sigma)-p_{3}(t, \sigma)\right| \\
& \quad \leq \int_{0}^{\gamma^{-1}(\gamma(t)-\sigma)}|\chi(\sigma-\gamma(t+h)+\gamma(u))-\chi(\sigma-\gamma(t)+\gamma(u))| d u \\
& \quad+\int_{\gamma^{-1}(\gamma(t)-\sigma)}^{\gamma^{-1}(\gamma(t+h)-\sigma)} \chi(\sigma-\gamma(t+h)+\gamma(u)) d u
\end{aligned}
$$

so that, with the change of variable $v=\sigma+\gamma(u)$,

$$
\begin{aligned}
& \left\|p_{3}(t+h, \cdot)-p_{3}(t, \cdot)\right\|_{L^{\infty}} \\
& \quad \leq \frac{1}{m_{\dot{\gamma}}}\left(\int_{\mathbb{R}}|\chi(v-\gamma(t+h))-\chi(v-\gamma(t))| d v+\int_{\gamma(t)}^{\gamma(t+h)} d v\right) \\
& \quad \leq \frac{3}{m_{\dot{\gamma}}}(\gamma(t+h)-\gamma(t)) .
\end{aligned}
$$

This concludes the proof of the continuity in time of $p$, with values in $L^{1}$.

Step 3: Expression (3.4) satisfies 1.1. We first show that $p$ defined by (3.4) satisfies

$$
\frac{\partial p}{\partial t}+\dot{\gamma}(t) \frac{\partial p}{\partial \sigma}=-\chi p+\phi \delta_{0}(\sigma) \quad \text { in } \mathcal{D}^{\prime}((0, T) \times \mathbb{R})
$$


For all $\psi \in \mathcal{D}((0, T) \times \mathbb{R})$,

$$
\begin{aligned}
& -\int_{0}^{T} \int_{\mathbb{R}} p\left(\frac{\partial \psi}{\partial t}+\dot{\gamma} \frac{\partial \psi}{\partial \sigma}-\chi \psi\right) \\
& =-\int_{0}^{T} \int_{\mathbb{R}} p_{0}(\sigma-\gamma(t)) \mathrm{e}^{-\int_{0}^{t} \chi(\sigma-\gamma(t)+\gamma(u)) d u}\left(\frac{\partial \psi}{\partial t}+\dot{\gamma} \frac{\partial \psi}{\partial \sigma}-\chi \psi\right)(t, \sigma) d \sigma d t \\
& -\int_{0}^{T} \int_{0}^{\gamma(t)} \frac{\phi \circ \gamma^{-1}(\gamma(t)-\sigma)}{\dot{\gamma} \circ \gamma^{-1}(\gamma(t)-\sigma)} \mathrm{e}^{-\int_{\gamma^{-1}(\gamma(t)-\sigma)}^{t} \chi(\sigma-\gamma(t)+\gamma(u)) d u} \\
& \times\left(\frac{\partial \psi}{\partial t}+\dot{\gamma} \frac{\partial \psi}{\partial \sigma}-\chi \psi\right)(t, \sigma) d \sigma d t \\
& =-\int_{0}^{T} \int_{\mathbb{R}} p_{0}(\xi) \mathrm{e}^{-\int_{0}^{t} \chi(\xi+\gamma(u)) d u}\left(\frac{\partial \psi}{\partial t}+\dot{\gamma} \frac{\partial \psi}{\partial \sigma}-\chi \psi\right)(t, \xi+\gamma(t)) d \xi d t \\
& -\int_{0}^{T} \int_{0}^{t} \phi(v) \mathrm{e}^{-\int_{v}^{t} \chi(-\gamma(v)+\gamma(u)) d u} \\
& \times\left(\frac{\partial \psi}{\partial t}+\dot{\gamma} \frac{\partial \psi}{\partial \sigma}-\chi \psi\right)(t,-\gamma(v)+\gamma(t)) d v d t
\end{aligned}
$$

where we have made the changes of variables $\xi=\sigma-\gamma(t)$ and $v=\gamma^{-1}(\gamma(t)-\sigma)$. Introducing

$$
\begin{aligned}
\nu(t, \xi) & =\psi(t, \xi+\gamma(t)) \mathrm{e}^{-\int_{0}^{t} \chi(\xi+\gamma(u)) d u} \\
\text { and } \mu(t, v) & =\psi(t,-\gamma(v)+\gamma(t)) \mathrm{e}^{-\int_{v}^{t} \chi(-\gamma(v)+\gamma(u)) d u}
\end{aligned}
$$

this rewrites

$$
\begin{aligned}
& -\int_{0}^{T} \int_{\mathbb{R}} p\left(\frac{\partial \psi}{\partial t}+\dot{\gamma} \frac{\partial \psi}{\partial \sigma}-\chi \psi\right) \\
& =-\int_{\mathbb{R}} p_{0}(\xi)\left[\int_{0}^{T} \frac{\partial \nu}{\partial t}(t, \xi) d t\right] d \xi-\int_{0}^{T} \phi(v)\left[\int_{v}^{T} \frac{\partial \mu}{\partial t}(t, v) d t\right] d v \\
& =\int_{\mathbb{R}} p_{0}(\xi)[\nu(0, \xi)-\nu(T, \xi)] d \xi \\
& \quad+\int_{0}^{T} \phi(v)[\mu(v, v)-\mu(T, v)] d v=\int_{0}^{T} \phi(v) \psi(v, 0) d v
\end{aligned}
$$

thus 3.9 .

We finally show that $\phi=\int \chi p$ a.e. on $(0, T)$, where $p$ is defined by (3.4). This will prove that 1.1 holds in $D^{\prime}((0, T) \times \mathbb{R})$. First, for almost all $t \in\left(0, \gamma^{-1}\left(\sigma_{c}\right)\right)$, definition (3.4) of $p$ implies $\int \chi(\sigma) p(t, \sigma) d \sigma=A(t)$. The definition (3.3) of $\phi$ implies $\phi(t)=A(t)$, thus $\phi(t)=\int \chi(\sigma) p(t, \sigma) d \sigma$ for such a time $t$. We next take $t \in$ 
$\left(\gamma^{-1}\left(\sigma_{c}\right), T\right)$. We have

$$
\begin{aligned}
f(t) & =A(t)+\int_{\sigma_{c}}^{\gamma(t)} \frac{\phi \circ \gamma^{-1}(\gamma(t)-\sigma)}{\dot{\gamma} \circ \gamma^{-1}(\gamma(t)-\sigma)} \mathrm{e}^{-\int_{\gamma^{-1}(\gamma(t)-\sigma)}^{t} \chi(\sigma-\gamma(t)+\gamma(u)) d u} d \sigma \\
& =A(t)+\int_{\sigma_{c}}^{\gamma(t)} \frac{\phi \circ \gamma^{-1}(\gamma(t)-\sigma)}{\dot{\gamma} \circ \gamma^{-1}(\gamma(t)-\sigma)} \mathrm{e}^{-t+\gamma^{-1}\left(\gamma(t)-\sigma+\sigma_{c}\right)} d \sigma \\
& =A(t)+\int_{0}^{\gamma^{-1}\left(\gamma(t)-\sigma_{c}\right)} \phi(s) \mathrm{e}^{-t+\gamma^{-1}\left(\gamma(s)+\sigma_{c}\right)} d s \\
& =\phi(t),
\end{aligned}
$$

where we have respectively simplified the exponential term, made the change of variables $s=\gamma^{-1}(\gamma(t)-\sigma)$ and used the definition (3.3) of $\phi$. This concludes the proof of this step, and thus that of Theorem 3.1 .

4. Properties of the solution. In this section we prove various properties of the solution of (1.1) the existence and uniqueness of which has been established in Theorem 3.1. We therefore assume throughout this section that, as for Theorem 3.1. $p_{0}$ in $L^{1}(\mathbb{R})$ and $\dot{\gamma}$ satisfies 3.1 .

Lemma 4.1 (Maximum principle). If $p_{0}(\sigma) \geq 0$ for almost all $\sigma \in \mathbb{R}$ then $p(t, \sigma) \geq 0$ for almost all $t \in[0, T), \sigma \in \mathbb{R}$.

Proof. Using the definition (3.2) of $A$, we first have $A(t) \geq 0$ for almost all $t \in[0, T)$. Because of recurrence relation $(3.3)$ on $\phi$, we then find that $\phi(t) \geq 0$ for almost all $t \in[0, T)$. Consequently, the expression (3.4) on $p$ gives the result.

Lemma 4.2 (Mass conservation). If $\int_{\mathbb{R}} p_{0}=1$ then $\int_{\mathbb{R}} p(t, \cdot)=1$ for all $t \in[0, T)$.

Proof. Denote

$$
h(t)=\int_{\mathbb{R}} p(t, \cdot)
$$

which is continuous since $p$ belongs to $C^{0}\left([0, T) ; L^{1}\right)$. Using (3.4), we obtain that $h$ reads

$$
\begin{aligned}
h(t)= & \int_{\mathbb{R}} p_{0}(\sigma-\gamma(t)) \mathrm{e}^{-\int_{0}^{t} \chi(\sigma-\gamma(t)+\gamma(u)) d u} d \sigma \\
& +\int_{0}^{\gamma(t)} \frac{\phi \circ \gamma^{-1}(\gamma(t)-\sigma)}{\dot{\gamma} \circ \gamma^{-1}(\gamma(t)-\sigma)} \mathrm{e}^{-\int_{\gamma^{-1}(\gamma(t)-\sigma)}^{t} \chi(\sigma-\gamma(t)+\gamma(u)) d u} d \sigma .
\end{aligned}
$$

For all $t \in\left[0, \gamma^{-1}\left(\sigma_{c}\right)\right], h$ rewrites

$$
\begin{aligned}
h(t) & =\int_{\mathbb{R}} p_{0}(\xi) \mathrm{e}^{-\int_{0}^{t} \chi(\xi+\gamma(u)) d u} d \xi+\int_{0}^{\gamma(t)} \frac{\phi \circ \gamma^{-1}(\gamma(t)-\sigma)}{\dot{\gamma} \circ \gamma^{-1}(\gamma(t)-\sigma)} d \sigma \\
& =\int_{\mathbb{R}} p_{0}(\xi) \mathrm{e}^{-\int_{0}^{t} \chi(\xi+\gamma(u)) d u} d \xi+\int_{0}^{t} \phi(s) d s .
\end{aligned}
$$

Differentiating $h$ in the sense of distributions in time, we obtain

$$
\begin{aligned}
\dot{h}(t) & =-\int_{\mathbb{R}} \chi(\xi+\gamma(t)) p_{0}(\xi) \mathrm{e}^{-\int_{0}^{t} \chi(\xi+\gamma(u)) d u} d \xi+\phi(t) \\
& =-A(t)+\phi(t) \\
& =0
\end{aligned}
$$


using the definitions 3.2 of $A$ and $(3.3)$ of $\phi$. Using that $h$ is continuous on $[0, T)$ with $h(0)=1$, we find $h=1$ on $\left[0, \gamma^{-1}\left(\sigma_{c}\right)\right]$. For all $t \in\left(\gamma^{-1}\left(\sigma_{c}\right), T\right), h$ rewrites

$$
\begin{aligned}
h(t)= & \int_{\mathbb{R}} p_{0}(\xi) \mathrm{e}^{-\int_{0}^{t} \chi(\xi+\gamma(u)) d u} d \xi+\int_{0}^{\sigma_{c}} \frac{\phi \circ \gamma^{-1}(\gamma(t)-\sigma)}{\dot{\gamma} \circ \gamma^{-1}(\gamma(t)-\sigma)} d \sigma \\
& +\int_{\sigma_{c}}^{\gamma(t)} \frac{\phi \circ \gamma^{-1}(\gamma(t)-\sigma)}{\dot{\gamma} \circ \gamma^{-1}(\gamma(t)-\sigma)} \mathrm{e}^{-t+\gamma^{-1}\left(\gamma(t)-\sigma+\sigma_{c}\right)} d \sigma \\
= & \int_{\mathbb{R}} p_{0}(\xi) \mathrm{e}^{-\int_{0}^{t} \chi(\xi+\gamma(u)) d u} d \xi+\int_{\gamma^{-1}\left(\gamma(t)-\sigma_{c}\right)}^{t} \phi(s) d s \\
& +\int_{0}^{\gamma^{-1}\left(\gamma(t)-\sigma_{c}\right)} \phi(s) \mathrm{e}^{-t+\gamma^{-1}\left(\gamma(s)+\sigma_{c}\right)} d s .
\end{aligned}
$$

Differentiating $h$ in $\mathcal{D}^{\prime}\left(\gamma^{-1}\left(\sigma_{c}\right), T\right)$, we obtain

$$
\begin{aligned}
\dot{h}(t) & =-A(t)+\phi(t)-\int_{0}^{\gamma^{-1}\left(\gamma(t)-\sigma_{c}\right)} \phi(s) \mathrm{e}^{-t+\gamma^{-1}\left(\gamma(s)+\sigma_{c}\right)} d s \\
& =0
\end{aligned}
$$

because of definitions (3.2) of $A$ and $(3.3)$ of $\phi$. This implies $h=1$ on $[0, T)$, hence the result.

Lemma 4.3 ( $L^{\infty}$-bounds). Assume $p_{0}$ satisfies

$$
p_{0} \in L^{1}(\mathbb{R}), \quad p_{0} \geq 0 .
$$

Then, $\phi$ defined by (3.3) (or, equivalently, $f$, given (3.5) satisfies

$$
\|\phi\|_{L^{\infty}(0, T)} \leq \int_{\mathbb{R}} p_{0} .
$$

If in addition $p_{0}$ satisfies

$$
p_{0} \in L^{\infty}(\mathbb{R}),
$$

then $p$ belongs to $L^{\infty}\left(0, T ; L^{\infty}\right)$ and there exists a constant $C_{\infty}$ which depends only on $\left\|p_{0}\right\|_{L^{1} \cap L^{\infty}}$ and the bound $m_{\dot{\gamma}}$ in (3.1) such that

$$
\|p\|_{L_{T}^{\infty}\left(L^{\infty}\right)} \leq C_{\infty}
$$

Proof. First, we notice that

$$
f(t)=\int_{\mathbb{R}} \chi p(t, \cdot) \leq \int_{\mathbb{R}} p(t, \cdot)=\int_{\mathbb{R}} p_{0},
$$

successively using the definition 1.4 of $f$, Lemma 4.1 and Lemma 4.2 . This immediately implies

$$
\|\phi\|_{L^{\infty}(0, T)} \leq \int_{\mathbb{R}} p_{0}
$$


Using the lower bound (3.1) on $\dot{\gamma}$, the $L^{\infty}$-bound on $p_{0}$ and the expression (3.4) of $p$ in terms of $\phi$, we find that $p$ belongs to $L^{\infty}\left(0, T ; L^{\infty}\right)$ and

$$
\|p\|_{L_{T}^{\infty}\left(L^{\infty}\right)} \leq\left\|p_{0}\right\|_{L^{\infty}}+\frac{\int_{\mathbb{R}} p_{0}}{m_{\dot{\gamma}}} .
$$

Lemma 4.4 (Delay differential equation). Assume $p_{0} \in L^{\infty}(\mathbb{R}) \cap L^{1}(\mathbb{R})$. Then, $A$ and $\phi=f$ respectively defined by $(3.2)$, (3.3) and $(1.4)$, belong to $W^{1,1}(0, T)$ thus are continuous in time. Moreover, $A$ and $\phi$ satisfy, for almost all $t \in(0, T)$,

$$
\begin{aligned}
\dot{A}(t)+A(t)=\dot{\gamma}(t) & {\left[p_{0}\left(\sigma_{c}-\gamma(t)\right) \mathrm{e}^{-\int_{0}^{t} \chi\left(\sigma_{c}-\gamma(t)+\gamma(u)\right) d u}\right.} \\
& \left.-p_{0}\left(-\sigma_{c}-\gamma(t)\right) \mathrm{e}^{-\int_{0}^{t} \chi\left(-\sigma_{c}-\gamma(t)+\gamma(u)\right) d u}\right]
\end{aligned}
$$

and for almost all $t \in\left(\gamma^{-1}\left(\sigma_{c}\right), T\right)$,

$$
\dot{\phi}(t)+\phi(t)-\frac{\dot{\gamma}(t)}{\dot{\gamma} \circ \gamma^{-1}\left(\gamma(t)-\sigma_{c}\right)} \phi \circ \gamma^{-1}\left(\gamma(t)-\sigma_{c}\right)=\dot{A}(t)+A(t) .
$$

Proof. As stated in Theorem 3.1, the functions $A$ and $\phi$ belong to $L^{\infty}(0, T) \subset$ $L^{1}(0, T)$. We show that both $\dot{A}$ and $\phi$ belong to $L^{1}(0, T)$.

The expression $(3.2)$ on $A$ rewrites

$$
A(t)=\int_{\mathbb{R} \backslash\left[-\sigma_{c}-\gamma(t), \sigma_{c}-\gamma(t)\right]} p_{0}(\xi) \mathrm{e}^{-\int_{0}^{t} \chi(\xi+\gamma(u)) d u} d \xi .
$$

Differentiating the above expression in the sense of distributions in time, we obtain equation 4.5. Since $p_{0}$ belongs to $L^{\infty}(0, T)$ and $\dot{\gamma}$ and $A$ belong to $L^{1}(0, T), \dot{A}$ belongs to $L^{1}(0, T)$ and the equation 4.5 holds for almost all $t \in(0, T)$.

Since $\phi=A$ on $\left(0, \gamma^{-1}\left(\sigma_{c}\right)\right), \dot{\phi}$ belongs to $L^{1}\left(0, \gamma^{-1}\left(\sigma_{c}\right)\right)$. Differentiating the recurrence relation (3.3) in the sense of distributions on $\left(\gamma^{-1}\left(\sigma_{c}\right), T\right)$, we have

$$
\begin{aligned}
\dot{\phi}(t)= & \dot{A}(t)+\frac{\dot{\gamma}(t)}{\dot{\gamma} \circ \gamma^{-1}\left(\gamma(t)-\sigma_{c}\right)} \phi \circ \gamma^{-1}\left(\gamma(t)-\sigma_{c}\right) \\
& -\int_{0}^{\gamma^{-1}\left(\gamma(t)-\sigma_{c}\right)} \phi(s) \mathrm{e}^{-t+\gamma^{-1}\left(\gamma(s)+\sigma_{c}\right)} d s, \\
= & \dot{A}(t)+\frac{\dot{\gamma}(t)}{\dot{\gamma} \circ \gamma^{-1}\left(\gamma(t)-\sigma_{c}\right)} \phi \circ \gamma^{-1}\left(\gamma(t)-\sigma_{c}\right)+A(t)-\phi(t) .
\end{aligned}
$$

Using that $A, \dot{\gamma}$ and $\phi$ respectively belong to $W^{1,1}(0, T), L^{1}(0, T)$ and $L^{\infty}(0, T)$, the right-hand side of the above equation and thus $\dot{\phi}$ belong to $L^{1}\left(\gamma^{-1}\left(\sigma_{c}\right), T\right)$. Moreover, the equation 4.6 holds for almost all $t \in\left(\gamma^{-1}\left(\sigma_{c}\right), T\right)$. This ends the proof.

Lemma 4.5 (Existence of $\tau$ ). Assume that $p_{0}$ satisfies

$$
p_{0} \in L^{1}(\mathbb{R}), \quad p_{0} \geq 0 \quad \int|\sigma| p_{0}<\infty .
$$


Then $\sigma p$ belongs to $L^{\infty}\left(0, T ; L^{1}\right)$ so that the average stress $\tau$ defined by 1.5 belongs to $L^{\infty}(0, T)$. Moreover, if there exists a scalar $M_{\dot{\gamma}}$ independent from $T$ such that $\dot{\gamma} \leq M_{\dot{\gamma}}$, then there exists a constant $C_{\tau}$ independent from $T$ such that

$$
\left\|\int|\sigma| p(t, \sigma) d \sigma\right\|_{L^{\infty}(0, T)} \leq C_{\tau}\left(1+M_{\dot{\gamma}} T\right) .
$$

Proof. We multiply the expression (3.4) on $p$ by $|\sigma|$ and integrate in $\sigma$. This implies

$$
\begin{aligned}
& \int|\sigma| p(t, \sigma) d \sigma \\
& \leq \int|\sigma| p_{0}(\sigma-\gamma(t)) d \sigma+\int_{0}^{\gamma(t)} \sigma \frac{\|\phi\|_{L^{\infty}}}{m_{\dot{\gamma}}} \mathrm{e}^{-\int_{\gamma^{-1}(\gamma(t)-\sigma)}^{t} \chi(\sigma-\gamma(t)+\gamma(u)) d u} d \sigma
\end{aligned}
$$

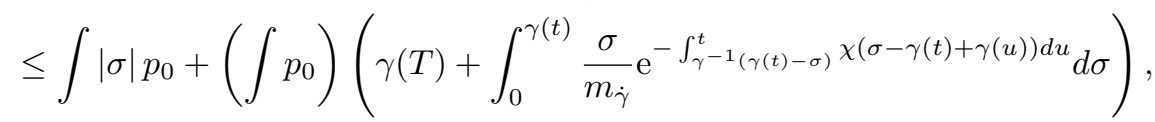

using that $\dot{\gamma} \geq m_{\dot{\gamma}}$ and then the upper bound (4.2) on $\|\phi\|_{L^{\infty}(0, T)}$. We deduce

$$
\int|\sigma| p(t, \sigma) d \sigma \leq \int|\sigma| p_{0}+\left(\int p_{0}\right)\left(\gamma(T)+\int_{0}^{\gamma(T)} \frac{\sigma}{m_{\dot{\gamma}}} d \sigma\right),
$$

so that $\sigma p$ belongs to $L^{\infty}\left(0, T ; L^{1}\right)$. Moreover, from (4.8), we obtain, for all $t>$ $\gamma^{-1}\left(\sigma_{c}\right)$

$$
\begin{aligned}
\int|\sigma| p(t, \sigma) d \sigma \leq & \max \left(\int p_{0}, \int|\sigma| p_{0}\right) \\
& \left(1+M_{\dot{\gamma}} T+\int_{0}^{\sigma_{c}} \frac{\sigma}{m_{\dot{\gamma}}} d \sigma+\frac{1}{m_{\dot{\gamma}}} \int_{\sigma_{c}}^{\gamma(t)} \sigma \mathrm{e}^{-t+\gamma^{-1}\left(\gamma(t)-\sigma+\sigma_{c}\right)} d \sigma\right) .
\end{aligned}
$$

Additionally, with the change of variable $v=t-\gamma^{-1}\left(\gamma(t)-\sigma+\sigma_{c}\right)$, the last integral satisfies

$$
\begin{aligned}
\int_{\sigma_{c}}^{\gamma(t)} \sigma \mathrm{e}^{-t+\gamma^{-1}\left(\gamma(t)-\sigma+\sigma_{c}\right)} d \sigma & =\int_{0}^{t-\gamma^{-1}\left(\sigma_{c}\right)}\left(\gamma(t)-\gamma(t-v)+\sigma_{c}\right) \mathrm{e}^{-v} \frac{1}{\dot{\gamma} \circ \gamma^{-1}\left(\gamma(t)-\sigma+\sigma_{c}\right)} d v \\
& \leq \int_{0}^{t-\gamma^{-1}\left(\sigma_{c}\right)}\left(\int_{t-v}^{t} M_{\dot{\gamma}} d u+\sigma_{c}\right) \frac{\mathrm{e}^{-v}}{m_{\dot{\gamma}}} d v \\
& \leq \int_{0}^{\infty}\left(M_{\dot{\gamma}} v+\sigma_{c}\right) \frac{\mathrm{e}^{-v}}{m_{\dot{\gamma}}} d v<\infty .
\end{aligned}
$$

Inserting the above inequality in 4.9 yields 4.7 . This concludes the proof.

Throughout our article, we now assume that

$$
p_{0} \in L^{1}(\mathbb{R}) \cap L^{\infty}(\mathbb{R}), \quad p_{0} \geq 0, \quad \int_{\mathbb{R}} p_{0}=1 \quad \text { and } \int_{\mathbb{R}}|\sigma| p_{0}<\infty,
$$

so that the five Lemmata $4.1,4.2,4.3,4.4$ and 4.5 hold. 
5. Longtime behavior in the case $\dot{\gamma}(t)=\dot{\gamma}_{\infty}$. In this section, we assume that $\dot{\gamma}(t)=\dot{\gamma}_{\infty}$ where $\dot{\gamma}_{\infty}>0$ is a given fixed constant and we study the longtime convergence for equation (1.1). This allows us to lay some ground work which will prove useful for the general case of a slowly varying shear rate addressed in the next sections. We prove the following.

Theorem 5.1. Assume that $\dot{\gamma}(t)=\dot{\gamma}_{\infty}$ with $\dot{\gamma}_{\infty}>0$ a given constant. Supply equation (1.1) with an initial condition $p_{0}$ that satisfies (4.10). Consider $p$ the solution to (1.1) and $p_{\infty}$ the associated stationary solution, the existence and uniqueness of those has been respectively established in Theorem 3.1 and Lemma 2.1. Then p converges exponentially fast in time to $p_{\infty}$ for almost all $\sigma \in \mathbb{R}$. In addition, $f$ defined by (1.4) converges exponentially fast in time to $\int \chi p_{\infty}$.

More precisely, there exist $b, C_{1}>0$ such that, for all $t$ and for almost all $\sigma \in \mathbb{R}$,

$$
\left|p(t, \sigma)-p_{\infty}(\sigma)\right| \leq C_{1}\left(\mathrm{e}^{-t}+\left(\mathrm{e}^{-b t} \mathrm{e}^{b \frac{\sigma}{\dot{\gamma} \infty}}+\mathrm{e}^{-t} \mathrm{e}^{\frac{\sigma}{\dot{\gamma} \infty}}\right) \mathbb{1}_{\left(0, \dot{\gamma}_{\infty} t\right)}(\sigma)\right) .
$$

In addition, there exists a positive continuous function $C_{2}$ of $\omega \in \mathbb{R}_{+}^{*}$ such that for all $t \geq 0$,

$$
\left|f(t)-\int \chi p_{\infty}\right| \leq C_{2}(\omega)\left(\mathrm{e}^{-b t}+\mathrm{e}^{-t}\right) .
$$

In the two estimates (5.1) and (5.2), the rate $b>0$ can be chosen as

$$
b=-\max \left\{x<0 \text { s.t. } x+1-\mathrm{e}^{-\omega x} \cos \left(\omega \sqrt{\mathrm{e}^{-2 \omega x}-(x+1)^{2}}\right)=0\right\}-\eta
$$

for any $\eta>0$, where $\omega$ is defined by:

$$
\omega=\frac{\sigma_{c}}{\dot{\gamma}_{\infty}} .
$$

Notice that the estimates (5.1) and (5.2) rely on the two natural timescales of the original problem: the exponential rate 1 of the jump process to zero (the hidden coefficient 1 multiplying the right-hand side of the Fokker-Planck equation (1.1)), and the typical time $\omega$ required for the process to leave the domain $\left(-\sigma_{c}, \sigma_{c}\right)$, when the shear rate is $\dot{\gamma}_{\infty}$.

Let us notice from 5.3 that we may assume in the following, without loss of generality, that

$$
b \neq 1 .
$$

This will be an assumption on $b$ in the forthcoming sections. This assumption is exclusively technical. We require it to simplify some proofs where convolutions with exponential kernels are involved, in particular to use estimates such as $\int_{0}^{t} \mathrm{e}^{-(t-s)} \mathrm{e}^{-b s} d s \leq$ $C\left(\mathrm{e}^{-b t}+\mathrm{e}^{-t}\right)$ (which is only true if $b \neq 1$ ).

Before we get to the proof of Theorem 5.1, to which the rest of this section is devoted, we need to introduce some notation and make some preliminaries.

Denote by $q(t, \sigma)=p(t, \sigma)-p_{\infty}(\sigma)$ and $q_{0}(\sigma)=p_{0}(\sigma)-p_{\infty}(\sigma)$. By linearity, and in place of $A$ and $\phi$ introduced in Theorem 3.1, we similarly introduce

$$
B(t)=\int \chi\left(\xi+\dot{\gamma}_{\infty} t\right) q_{0}(\xi) \mathrm{e}^{-\frac{1}{\dot{\gamma} \infty} \int_{\xi}^{\xi+\dot{\gamma}_{\infty} t} \chi(v) d v} d \xi
$$


and

$$
g(t)= \begin{cases}B(t) & \text { for almost all } t \in(0, \omega) \\ B(t)+\int_{0}^{t-\omega} g(s) \mathrm{e}^{-t+s+\omega} d s & \text { for almost all } t \in(k \omega,(k+1) \omega) .\end{cases}
$$

We then have from Theorem 3.1

$$
q(t, \sigma)=q_{0}\left(\sigma-\dot{\gamma}_{\infty} t\right) \mathrm{e}^{-\frac{1}{\dot{\gamma}_{\infty}} \int_{\sigma-\dot{\gamma}_{\infty} t}^{\sigma} \chi(v) d v}+\frac{1}{\dot{\gamma}_{\infty}} g\left(t-\frac{\sigma}{\dot{\gamma}_{\infty}}\right) \mathrm{e}^{-\frac{1}{\dot{\gamma}_{\infty}} \int_{0}^{\sigma} \chi(v) d v} \mathbb{1}_{\left(0, \dot{\gamma}_{\infty} t\right)}(\sigma) .
$$

Using Lemma 4.4 $g$ satisfies, for almost all $t>\omega$,

$$
\dot{g}(t)+g(t)-g(t-\omega)=\dot{B}(t)+B(t) .
$$

The equation (5.9) on $g$ is a delay differential equation with constant coefficients. The proof of Theorem 5.1 is based upon three lemmata for such a delay differential equation, denoted in generality by

$$
\left\{\begin{aligned}
\dot{u}(t)+u(t)-u(t-\omega) & =\mu(t) \text { for } t \geq \omega \\
u(t) & =\nu(t) \text { for } t \in(0, \omega)
\end{aligned}\right.
$$

where $\omega>0$ is a constant and $\mu$ is a locally integrable function. Equation (5.10) is understood in the sense of distribution in time. To such a delay differential equation is classically associated the unique function $k(t)$ satisfying the following properties:

1. $k(t)=0, \quad \forall t<0$;

2. $k(0)=1$;

3. $k(t)$ is continuous on $[0, \infty)$;

4. $k(t)$ satisfies for all $t>0$,

$$
\dot{k}(t)+k(t)-k(t-\omega)=0 .
$$

The three lemmata useful for the proof of Theorem 5.1 are Lemma 5.4. itself proved using Lemma 5.2 , and Lemma 5.3 . The latter two lemmata, Lemma 5.2 and Lemma 5.3, are borrowed respectively from [10, and 2]. They are valid for more general delay differential equations, but for simplicity, we state them here for our specific delay differential equation 5.10$)$.

Lemma 5.2. [10, Equation (5.10), p. 22] Consider $k$ defined by (5.11) and the associated properties above. Denote by

$$
h(\lambda)=\lambda+1-\mathrm{e}^{-\omega \lambda} .
$$

Then, for all $\alpha_{m} \in \mathbb{R}$ such that no root of $h$ has real part equal to $\alpha_{m}$, the function $k$ writes, for all $t>0$,

$$
k(t)=\sum_{j=1}^{k_{m}} \operatorname{Res}_{\lambda=\lambda_{j}}\left(\frac{\mathrm{e}^{\lambda t}}{h(\lambda)}\right)+\frac{1}{2 \pi i} \lim _{T \rightarrow \infty} \int_{-T}^{T} \frac{\mathrm{e}^{\left(\alpha_{m}+i u\right) t}}{h\left(\alpha_{m}+i u\right)} d u .
$$

where $\lambda_{1}, \ldots, \lambda_{k_{m}}$ are the roots of $h$ such that $\Re\left(\lambda_{j}\right)>\alpha_{m}$ and $\operatorname{Res}_{\lambda=\lambda_{j}}$ denotes the residue at $\lambda=\lambda_{j}$. 
Intuitively, (5.13) is obtained as follows. The function $k$ solves $\dot{k}(t)+k(t)-k(t-$ $\omega)=\delta_{0}$ on $\mathcal{D}^{\prime}(\mathbb{R})$, which by Laplace transform and using the notation 5.12 , yields $h(s) \mathcal{L}(k)(s)=1$ (where $\mathcal{L}(k)$ denotes the Laplace transform of $k$ ). It remains then to divide by $h$ and apply a reverse Laplace transform to finally obtain $k$. The difficulty is of course related to the zeros of the function $h$.

Lemma 5.3. [2, Theorem 3.7, p. 75] Consider two functions $\nu \in C^{0}[0, \omega]$ and $\mu \in L_{\mathrm{loc}}^{1}(\omega, \infty)$. Then, there exists a unique solution $u(t) \in C^{0}\left(\mathbb{R}_{+}\right)$verifying $(5.10)$ in the sense of distribution, and $u(t)$ satisfies: for $t \geq 0$,

$$
u(t)=\nu(\omega) k(t-\omega)-\int_{0}^{\omega} \nu\left(t_{1}\right) k\left(t-t_{1}-\omega\right) d t_{1}+\int_{\omega}^{t} \mu\left(t_{1}\right) k\left(t-t_{1}\right) d t_{1} .
$$

REMARK 3. The result of Lemma 5.3 is stated in [2] for $\mu$ continuous but holds for $\mu \in L_{\text {loc }}^{1}(\omega, \infty)$. Indeed, the existence of a unique solution is still valid in this more general setting (see [10, p.14]) and expression (5.14) satisfies (5.10) almost everywhere.

As announced above, we first use Lemma 5.2 to prove the following

Lemma 5.4. Assume

$$
m_{0}<\omega<M_{0} .
$$

Then there exist $b>0$ and $C_{0}>0$ which depend only on $m_{0}$ and $M_{0}$ and such that for all $t>0$

$$
k(t)=\frac{1}{1+\omega}+k_{1}(t)
$$

with

$$
\left|k_{1}(t)\right| \leq C_{0} \mathrm{e}^{-b t} .
$$

Moreover, $b$ can be chosen as (5.3), for any $\eta>0$.

We immediately emphasize that the point in Lemma 5.4 is to show that the prefactor $C_{0}$ and the exponent $b$ appearing in (5.17) do not depend on $\omega$ itself, but can be chosen locally uniformly, that is, depend only on the bounds $m_{0}$ and $M_{0}$ of the interval where $\omega$ lies. Proving (5.17) for a fixed $\omega$ is a simple consequence of the classical results contained e.g. in [2, 10].

REMARK 4. Using numerical experiments, we will show in Section 8 that the rate $b$ given by (5.3) for the estimation (5.17) is indeed sharp. It is interesting to note that our result 5.4 in the present section does not explicitly require such a sharpness. A simpler alternate proof (which we owe to one of the anonymous referees) shows a similar, however non sharp estimation. That proof is based on the observation $k_{1}(t)+\int_{t-\omega}^{t} k_{1}(s) d s=0$ which shows that the function $k_{1}$ necessarily cancels on any interval $(m \omega,(m+1) \omega)$. This leads to the following induction relation on the maximum value $M_{m}$ of $\left|k_{1}\right|$ on $(m \omega,(m+1) \omega)$

$$
M_{m+1} \leq \max \left(M_{m-1}, M_{m}\right)\left(1-\mathrm{e}^{-2 \omega}\right) .
$$


Indeed, denoting $t_{m} \in(m \omega, m \omega+\omega)$ a real such that $k_{1}\left(t_{m}\right)=0, k_{1}$ solution of (5.11) satisfies, for all $t \in\left(t_{m}, m \omega+2 \omega\right)$,

$$
\left|k_{1}(t)\right|=\left|\int_{t_{m}}^{t} \mathrm{e}^{s-t} k_{1}(s-t) d s\right| \leq \max \left(M_{m-1}, M_{m}\right) \int_{t_{m}}^{t} \mathrm{e}^{s-t} d s
$$

hence (5.18). Denote $\lfloor$.$\rfloor the integer part. The induction relation (5.18) then implies$

$$
M_{m} \leq \max \left(M_{0}, M_{1}\right)\left(1-\mathrm{e}^{-2 \omega}\right)^{\left\lfloor\frac{m}{2}\right\rfloor}
$$

and therefore, using that for all $t \in(0,2 \omega), k_{1}(t)=\mathrm{e}^{-t}+\mathrm{e}^{\omega-t}(t-\omega) \mathbb{1}_{[\omega, 2 \omega]}(t)-\frac{1}{1+\omega}$,

$$
\left|k_{1}(t)\right| \leq \tilde{C}_{0}(\omega) \mathrm{e}^{-\tilde{b}(\omega) t}
$$

where $\tilde{C}_{0}(\omega)=2+\omega$ and $\tilde{b}(\omega)=-\frac{\log \left(1-\mathrm{e}^{-2 \omega}\right)}{2 \omega}$ are respectively decreasing and increasing functions of $\omega$. This proves Lemma 5.4. with the values $b=\tilde{b}\left(M_{0}\right)$ and $C_{0}=\tilde{C}_{0}\left(M_{0}\right)$.

Proof of Lemma 5.4. The proof falls in three steps. We first derive an upper bound on the real part of the nonzero roots of the function $h$ defined by $(5.12)$. This upper bound actually yields the exponent $b$ in the exponential estimates of Lemma 5.4 and thus of Theorem5.1. In the second step, we apply Lemma 5.2. In the third and final step, we conclude.

Step 1: Upper bound on the real part of the nonzero roots of $h$. The roots of the function $h$ defined by (5.12) are 0 and the complex numbers $\lambda=\alpha+i \beta$ (with $\alpha, \beta \in \mathbb{R})$ that satisfy

$$
\begin{aligned}
\alpha+1 & =\mathrm{e}^{-\omega \alpha} \cos (\omega \beta), \\
\beta & =-\mathrm{e}^{-\omega \alpha} \sin (\omega \beta) .
\end{aligned}
$$

It is easy to check that $\alpha=0$ implies $\beta=0$ and conversely, so that in the following, we assume $\alpha \neq 0$ and $\beta \neq 0$. The equation $\left(5.20\right.$ rewrites $-\frac{\sin (\omega \beta)}{\beta}=\mathrm{e}^{\omega \alpha}$. Since the function $x \mapsto-\frac{\sin (\omega x)}{x}$ is non-positive on $\left[-\frac{\pi}{\omega}, \frac{\pi}{\omega}\right], \beta$ satisfies

$$
|\beta|>\frac{\pi}{\omega} .
$$

Moreover, we combine (5.19) and (5.20) and obtain

$$
\mathrm{e}^{2 \omega \alpha}\left((\alpha+1)^{2}+\beta^{2}\right)=1 .
$$

This implies that $\alpha$ is negative and therefore, using the bounds 5.15 and 5.21 respectively on $\omega$ and $\beta$,

$$
\mathrm{e}^{2 M_{0} \alpha}\left((\alpha+1)^{2}+\frac{\pi^{2}}{M_{0}^{2}}\right)<1
$$

The function

$$
\zeta: x \mapsto \mathrm{e}^{2 M_{0} x}\left((x+1)^{2}+\frac{\pi^{2}}{M_{0}^{2}}\right)
$$


is continuous, satisfies $\zeta(0)>1$ and $\lim _{-\infty} \zeta=0$ so that by the intermediate value theorem, there exists

$$
b>0 \text { such that } \zeta(-b)=1 \text { and } \zeta(x) \geq 1 \text { on }[-b, 0] .
$$

The scalar $b>0$ depends only on $M_{0}$. Additionally, the real part $\alpha$ of the nonzero roots of $h$ satisfies

$$
\alpha<-b
$$

and, combining (5.19) and 5.22,

$$
\alpha+1-\mathrm{e}^{-\omega \alpha} \cos \left(\omega \sqrt{\mathrm{e}^{-2 \omega \alpha}-(\alpha+1)^{2}}\right)=0
$$

Therefore $b$ can be chosen as (5.3), for any $\eta>0$.

Step 2: Applying Lemma 5.2. From the previous step, we know that the only root of $h$ with real part strictly above $-b$ is 0 . We now apply Lemma 5.2 with $\alpha_{m}=-b$. Since the root 0 is a simple root of $h$, the residue of $\frac{\mathrm{e}^{\lambda t}}{h(\lambda)}$ at 0 is $\frac{1}{\dot{h}(0)}=\frac{1}{1+\omega}$. Equation 5.13 therefore writes

$$
k(t)=\frac{1}{1+\omega}+\frac{1}{2 \pi i} \lim _{T \rightarrow \infty} \int_{-T}^{T} \frac{\mathrm{e}^{(-b+i u) t}}{h(-b+i u)} d u .
$$

Proving that there exists $C_{0}>0$ which depends only on $m_{0}$ and $M_{0}$, such that for all $t>0$,

$$
\lim _{T \rightarrow \infty}\left|\int_{-T}^{T} \frac{\mathrm{e}^{(-b+i u) t}}{h(-b+i u)} d u\right| \leq C_{0} \mathrm{e}^{-b t}
$$

therefore amounts to concluding the proof of Lemma 5.4. Actually, we will show that this holds up to changing $b$ to $b-\eta$ in the right hand side, for any positive $\eta$. This will conclude the proof.

Step 3: Exponential bound. We first show, for all $t>0$,

$$
\lim _{T \rightarrow \infty} \int_{-T}^{T} \frac{\mathrm{e}^{(-b+i u) t}}{h(-b+i u)} d u=\lim _{T \rightarrow \infty} \frac{1}{t} \int_{-T}^{T} \mathrm{e}^{(-b+i u) t} \frac{\dot{h}}{h^{2}}(-b+i u) d u .
$$

By integration by parts, we have

$$
\int_{-T}^{T} \frac{\mathrm{e}^{(-b+i u) t}}{h(-b+i u)} d u=\frac{1}{t} \int_{-T}^{T} \mathrm{e}^{(-b+i u) t} \frac{\dot{h}}{h^{2}}(-b+i u) d u+\frac{1}{i t}\left[\frac{\mathrm{e}^{(-b+i u) t}}{h(-b+i u)}\right]_{-T}^{T}
$$

Introduce $T_{0}>0$ such that for all $|T| \geq T_{0}$,

$$
\left(1+\frac{b^{2}}{T^{2}}\right)^{\frac{1}{2}}-\frac{1}{|T|}\left(1+\mathrm{e}^{M_{0} b}\right) \geq \frac{1}{2}
$$

Then, for all $|T|>T_{0}$,

$$
\begin{aligned}
|h(-b+i T)| & =\mid-b+i T+1-\mathrm{e}^{-\omega(-b+i T) \mid} \\
& \geq \sqrt{b^{2}+T^{2}}-1-\mathrm{e}^{\omega b} \geq \sqrt{b^{2}+T^{2}}-\left(1+\mathrm{e}^{M_{0} b}\right) \geq \frac{|T|}{2}
\end{aligned}
$$


so that, for all $t>0$,

$$
\left|\frac{1}{i t}\left[\frac{\mathrm{e}^{(-b+i u) t}}{h(-b+i u)}\right]_{-T}^{T}\right| \leq \frac{4 \mathrm{e}^{-b t}}{|T| t} .
$$

By passing to the limit $T \rightarrow \infty$ in (5.25), we thus obtain (5.24).

Now, for all $u \in \mathbb{R}$,

$$
\begin{aligned}
\left|\frac{\dot{h}}{h^{2}}\right|(-b+i u) & =\frac{\left|1+\omega \mathrm{e}^{-\omega(-b+i u)}\right|}{\left(1-b-\mathrm{e}^{\omega b} \cos (\omega u)\right)^{2}+\left(u+\mathrm{e}^{\omega b} \sin (\omega u)\right)^{2}} \\
& <\frac{1+M_{0} \mathrm{e}^{M_{0} b}}{(1-b)^{2}+u^{2}-2 \mathrm{e}^{\omega b}((1-b) \cos (\omega u)-u \sin (\omega u))} .
\end{aligned}
$$

Introduce $u_{0}>0$ which we may take depending only on $M_{0}$, such that for all $|u| \geq u_{0}$,

$$
(1-b)^{2}+u^{2}-2 \mathrm{e}^{M_{0} b}((1-b)+|u|)>0
$$

so that

$$
\left|\frac{\dot{h}}{h^{2}}\right|(-b+i u)<\frac{1+M_{0} \mathrm{e}^{M_{0} b}}{(1-b)^{2}+u^{2}-2 \mathrm{e}^{M_{0} b}((1-b)+|u|)} .
$$

For $T>u_{0}$ and $t>0$, this implies

$$
\frac{1}{t}\left|\int_{-T}^{T} \mathrm{e}^{(-b+i u) t} \frac{\dot{h}}{h^{2}}(-b+i u) d u\right|
$$

$$
\leq \frac{\mathrm{e}^{-b t}}{t}\left(\int_{-u_{0}}^{u_{0}}\left|\frac{\dot{h}}{h^{2}}\right|(-b+i u) d u+2 \int_{u_{0}}^{\infty} \frac{\left(1+M_{0} \mathrm{e}^{M_{0} b}\right) d u}{(1-b)^{2}+u^{2}-2 \mathrm{e}^{M_{0} b}((1-b)+|u|)}\right) .
$$

The function $\omega \mapsto \int_{-u_{0}}^{u_{0}}\left|\frac{\dot{h}}{h^{2}}\right|(-b+i u) d u$ is continuous for $\omega \in\left[m_{0}, M_{0}\right]$ and is therefore bounded by a constant that only depends on $m_{0}$ and $M_{0}$. From (5.24) and the bound (5.27), we deduce that there exists a constant $C_{0}>0$ that also only depends on $m_{0}$ and $M_{0}$ such that

$$
\lim _{T \rightarrow \infty}\left|\int_{-T}^{T} \frac{\mathrm{e}^{(-b+i u) t}}{h(-b+i u)} d u\right| \leq C_{0} \mathrm{e}^{-(b-\eta) t},
$$

for any positive $\eta$. This concludes the proof.

We are now in position to turn to the

Proof of Theorem 5.1. The proof proceeds in five steps. In step 1. we apply the above lemmata to the delay differential equation (5.9). In steps 2 and 3 , we derive some estimates that will be useful, in the last two steps, to show convergence of $g$, and eventually $q$. 
Step 1: Applying Lemmata 5.4 and 5.3. The function $g$ defined by 5.7 satisfies (5.10) in the particular case $\mu=\dot{B}+B$ and $\nu(t)=B(t)$. Notice that, by the Lemma 4.4 , the function $B$ defined by $(5.6)$ belongs to $W^{1,1}(0, T)$ and thus, in particular, is continuous in time. We apply Lemma 5.3 and find (by integration by parts): for all $t>\omega$,

$$
\begin{aligned}
g(t) & =B(\omega) k(t-\omega)+\int_{0}^{\omega} B\left(t_{1}\right) k\left(t-t_{1}-\omega\right) d t_{1}+\int_{\omega}^{t}(\dot{B}+B)\left(t_{1}\right) k\left(t-t_{1}\right) d t_{1} \\
& =B(\omega) k(t-\omega)+\int_{0}^{\omega} B\left(t_{1}\right)(\dot{k}+k)\left(t-t_{1}\right) d t_{1}+\int_{\omega}^{t}(\dot{B}+B)\left(t_{1}\right) k\left(t-t_{1}\right) d t_{1}
\end{aligned}
$$

$$
=B(0) k(t)+\int_{0}^{t}(\dot{B}+B)\left(t_{1}\right) k\left(t-t_{1}\right) d t_{1} .
$$

We now recall that, in this section, the value of $\omega$ is fixed by (5.4) at $\omega=\frac{\sigma_{c}}{\dot{\gamma}_{\infty}}$. We can apply Lemma 5.4 and insert the decomposition 5.16) of $k$ into the previous equation on $g$. We obtain

$$
\begin{aligned}
g(t)= & \frac{1}{1+\omega}\left(B(0)+\int_{0}^{t}(\dot{B}+B)\left(t_{1}\right) d t_{1}\right) \\
& +B(0) k_{1}(t)+\int_{0}^{t}(\dot{B}+B)\left(t_{1}\right) k_{1}\left(t-t_{1}\right) d t_{1} .
\end{aligned}
$$

where $k_{1}$ satisfies 5.17) with $b, C_{0}>0$ only depending on $\omega=\frac{\sigma_{c}}{\dot{\gamma}_{\infty}}$. Moreover, $b$ can be chosen as 5.3 for any positive $\eta$ as stated in Lemma 5.4

Our next two steps consist in deriving a couple of estimates (see (5.31) and (5.32) below) on the terms of 5.29 .

Step 2: Longtime convergence of $B(0)+\int_{0}^{t}(\dot{B}+B)\left(t_{1}\right) d t_{1}$. Using 4.5), the function $B$ defined by s.5.6) satisfies, for almost all $t>0$,

$$
\begin{aligned}
\dot{B}(t)+B(t)=\dot{\gamma}_{\infty}( & -q_{0}\left(-\sigma_{c}-\dot{\gamma}_{\infty} t\right) \mathrm{e}^{-\frac{1}{\dot{\gamma}_{\infty}} \int_{-\sigma_{c}-\dot{\gamma}_{\infty} t}^{-\sigma_{c}} \chi(\sigma) d \sigma} \\
& \left.+q_{0}\left(\sigma_{c}-\dot{\gamma}_{\infty} t\right) \mathrm{e}^{-\frac{1}{\dot{\gamma}_{\infty}} \int_{\sigma_{c}-\dot{\gamma}_{\infty} t}^{\sigma_{c}} \chi(\sigma) d \sigma}\right) .
\end{aligned}
$$

Computing $B(0)$ and integrating 5.30 from 0 to $t$ yield

$$
\begin{aligned}
B(0)+\int_{0}^{t}(\dot{B}+B)= & \int \chi q_{0}-\dot{\gamma}_{\infty} \int_{0}^{t} q_{0}\left(-\sigma_{c}-\dot{\gamma}_{\infty} t_{1}\right) \mathrm{e}^{-\frac{1}{\dot{\gamma}_{\infty}} \int_{-\sigma_{c}-\dot{\gamma}_{\infty} t_{1}}^{-\sigma_{c}} \chi(\sigma) d \sigma} d t_{1} \\
& +\dot{\gamma}_{\infty} \int_{0}^{t} q_{0}\left(\sigma_{c}-\dot{\gamma}_{\infty} t_{1}\right) \mathrm{e}^{-\frac{1}{\dot{\gamma}_{\infty}} \int_{\sigma_{c}-\dot{\gamma}_{\infty} t_{1}}^{\sigma_{c}} \chi(\sigma) d \sigma} d t_{1}
\end{aligned}
$$

so that, respectively with the changes of variables $v=-\sigma_{c}-\dot{\gamma}_{\infty} t$ and $v=\sigma_{c}-\dot{\gamma}_{\infty} t$ in the last two integrals, we obtain

$$
\begin{aligned}
B(0)+\int_{0}^{t}(\dot{B}+B)= & \int \chi q_{0}-\int_{-\sigma_{c}-\dot{\gamma}_{\infty} t}^{-\sigma_{c}} q_{0}(v) \mathrm{e}^{-\frac{1}{\dot{\gamma}_{\infty}} \int_{v}^{-\sigma_{c}} \chi(\sigma) d \sigma} d v \\
& +\int_{\sigma_{c}-\dot{\gamma}_{\infty} t}^{\sigma_{c}} q_{0}(v) \mathrm{e}^{-\frac{1}{\dot{\gamma}_{\infty}} \int_{v}^{\sigma_{c}} \chi(\sigma) d \sigma} d v .
\end{aligned}
$$


For $t>2 \omega$, this implies that

$$
\begin{aligned}
B(0)+\int_{0}^{t}(\dot{B}+B)\left(t_{1}\right) d t_{1}= & \int \chi q_{0}-\int_{-\sigma_{c}-\dot{\gamma}_{\infty} t}^{-\sigma_{c}} q_{0}(v) \mathrm{e}^{-\frac{1}{\dot{\gamma} \infty} \int_{v}^{-\sigma_{c}} \chi(\sigma) d \sigma} d v+\int_{-\sigma_{c}}^{\sigma_{c}} q_{0} \\
& +\int_{\sigma_{c}-\dot{\gamma}_{\infty} t}^{-\sigma_{c}} q_{0}(v) \mathrm{e}^{-\frac{1}{\dot{\gamma}_{\infty}} \int_{v}^{-\sigma_{c}} \chi(\sigma) d \sigma} d v \\
= & \int q_{0}-\int_{-\sigma_{c}-\dot{\gamma}_{\infty} t}^{\sigma_{c}-\dot{\gamma}_{\infty} t} q_{0}(v) \mathrm{e}^{\frac{\sigma_{c}+v}{\dot{\gamma} \infty}} d v \\
= & -\dot{\gamma}_{\infty} \int_{-\omega-t}^{\omega-t} q_{0}\left(\dot{\gamma}_{\infty} v\right) \mathrm{e}^{\omega+v} d v
\end{aligned}
$$

using that $\int q_{0}=0$. We deduce

$$
\left|B(0)+\int_{0}^{t}(\dot{B}+B)\left(t_{1}\right) d t_{1}\right| \leq \dot{\gamma}_{\infty}\left\|q_{0}\right\|_{L^{\infty}} \mathrm{e}^{2 \omega-t} .
$$

Step 3: Longtime convergence of $B(0) k_{1}(t)+\int_{0}^{t}(\dot{B}+B)\left(t_{1}\right) k_{1}\left(t-t_{1}\right) d t_{1}$. Using (5.30) on $\dot{B}+B$ and the estimate (5.17) on $k_{1}$, we have, for $t>2 \omega$,

$$
\begin{aligned}
\mid B(0) & k_{1}(t)+\int_{0}^{t}(\dot{B}+B)\left(t_{1}\right) k_{1}\left(t-t_{1}\right) d t_{1} \mid \\
\leq & C_{0}\left(\mathrm{e}^{-b t} \int \chi\left|q_{0}\right|+\int_{0}^{t} \dot{\gamma}_{\infty}\left|q_{0}\left(-\sigma_{c}-\dot{\gamma}_{\infty} t_{1}\right)\right| \mathrm{e}^{-t_{1}-b\left(t-t_{1}\right)} d t_{1}\right. \\
& \left.+\int_{0}^{t} \dot{\gamma}_{\infty}\left|q_{0}\left(\sigma_{c}-\dot{\gamma}_{\infty} t_{1}\right)\right| \mathrm{e}^{2 \omega} \mathrm{e}^{-t_{1}-b\left(t-t_{1}\right)} d t_{1}\right) \\
\leq & C_{0} \dot{\gamma}_{\infty}\left\|q_{0}\right\|_{L^{\infty}}\left(1+\mathrm{e}^{2 \omega}\right)\left(\mathrm{e}^{-b t}+\mathrm{e}^{-b t} \int_{0}^{t} \mathrm{e}^{(b-1) t_{1}} d t_{1}\right) \\
\leq & C_{0} \dot{\gamma}_{\infty}\left\|q_{0}\right\|_{L^{\infty}}\left(1+\mathrm{e}^{2 \omega}\right)\left(\frac{1}{|b-1|} \mathrm{e}^{-t}+\frac{b}{|b-1|} \mathrm{e}^{-b t}\right) .
\end{aligned}
$$

Here, we have used the assumption (5.5) on $b$.

Step 4: Longtime convergence of $g(t)$. Using the decomposition 5.29) and the estimates (5.31) and (5.32) derived in steps 2 and 3, we have, for $t>2 \omega$,

$$
|g(t)| \leq \dot{\gamma}_{\infty}\left\|q_{0}\right\|_{L^{\infty}}\left(\frac{\mathrm{e}^{2 \omega}}{1+\omega} \mathrm{e}^{-t}+C_{0}\left(1+\mathrm{e}^{2 \omega}\right)\left(\frac{1}{|b-1|} \mathrm{e}^{-t}+\frac{b}{|b-1|} \mathrm{e}^{-b t}\right)\right) .
$$

Recall that $g$ defined by (5.7) satisfies by linearity $g=\phi-\int \chi p_{\infty}$ and that $f=\phi$ a.e. (see (3.5)). We have obtained estimate (5.2) on $f-\int \chi p_{\infty}$.

Step 5: Longtime convergence of $q$. We now turn to $q(t, \sigma)=p(t, \sigma)-p_{\infty}(\sigma)$. Using (5.8), we have

$$
|q(t, \sigma)| \leq\left\|q_{0}\right\|_{L^{\infty}} \mathrm{e}^{2 \omega-t}+\frac{1}{\dot{\gamma}_{\infty}}\left|g\left(t-\frac{\sigma}{\dot{\gamma}_{\infty}}\right)\right| \mathbb{1}_{\left(0, \dot{\gamma}_{\infty} t\right)}(\sigma) .
$$

In view of the estimate 5.2 on $g$, we deduce, for almost all $\sigma \in \mathbb{R}$ and $t>0$,

$$
|q(t, \sigma)| \leq\left\|q_{0}\right\|_{L_{\infty}^{\infty}} \mathrm{e}^{2 \omega-t}+\frac{C_{2}}{\dot{\gamma}_{\infty}}\left(\mathrm{e}^{-\left(t-\frac{\sigma}{\dot{\gamma}_{\infty}}\right)}+\mathrm{e}^{-b\left(t-\frac{\sigma}{\dot{\gamma}_{\infty}}\right)}\right) \mathbb{1}_{\left(0, \dot{\gamma}_{\infty} t\right)}(\sigma) .
$$

This concludes the proof of Theorem 5.1 
6. Longtime convergence in the case $\dot{\gamma}(\epsilon t)$. Our main result in this section is the following.

THEOREM 6.1. Consider $\dot{\gamma}$ a Lipschitz function with Lipschitz constant $L_{\dot{\gamma}}$, which satisfies, for all $t \geq 0$,

$$
m_{\dot{\gamma}} \leq \dot{\gamma}(t) \leq M_{\dot{\gamma}}, \text { for some } m_{\dot{\gamma}}, M_{\dot{\gamma}}>0 \text { constant scalars }
$$

Consider an initial condition $p_{0}$ which satisfies 4.10 . For $\theta>0, \epsilon>0$ such that $\frac{\theta}{\epsilon}>2 \frac{\sigma_{c}}{m_{\dot{\gamma}}}$, consider the functions $p_{\epsilon}(t, \sigma)$ and $p_{\infty}(\theta, \sigma)$ respectively solutions to

$$
\begin{gathered}
\left\{\begin{array}{c}
\frac{\partial p_{\epsilon}}{\partial t}(t, \sigma)+\dot{\gamma}(\epsilon t) \frac{\partial p_{\epsilon}}{\partial \sigma}(t, \sigma)=-\chi(\sigma) p_{\epsilon}(t, \sigma)+\left(\int \chi(\sigma) p_{\epsilon}(t, \sigma) d \sigma\right) \delta_{0}(\sigma) \\
p_{\epsilon}(0, \sigma)=p_{0}(\sigma)
\end{array}\right. \\
\dot{\gamma}(\theta) \frac{\partial p_{\infty}(\theta, \sigma)}{\partial \sigma}=-\chi(\sigma) p_{\infty}(\theta, \sigma)+\left(\int \chi(\sigma) p_{\infty}(\theta, \sigma) d \sigma\right) \delta_{0}(\sigma)
\end{gathered}
$$

the existence and uniqueness of which have been respectively established in Theorem 3.1 and Lemma 2.1.

Then, there exist constants $b, C_{3}, C_{4}>0$ independent from $\theta$ and $\epsilon$ (satisfying $\left.\frac{\theta}{\epsilon}>2 \frac{\sigma_{c}}{m_{\dot{\gamma}}}\right)$ such that,

$$
\left|\int \chi(\sigma)\left(p_{\epsilon}\left(\frac{\theta}{\epsilon}, \sigma\right)-p_{\infty}(\theta, \sigma)\right) d \sigma\right| \leq C_{3}\left(\mathrm{e}^{-b \frac{\theta}{\epsilon}}+\mathrm{e}^{-\frac{\theta}{\epsilon}}+\epsilon\right)
$$

and, for almost all $\sigma \in \mathbb{R}$ such that $\sigma \leq \gamma\left(\frac{\theta}{\epsilon}\right)$,

$$
\left|p_{\epsilon}\left(\frac{\theta}{\epsilon}, \sigma\right)-p_{\infty}(\theta, \sigma)\right| \leq C_{4}\left[\left(\mathrm{e}^{-b \frac{\theta}{\epsilon}}+\mathrm{e}^{-\frac{\theta}{\epsilon}}+\epsilon\right)+\epsilon \mathbb{1}_{\left(\sigma_{c}, \infty\right)}(\sigma)\left(\sigma-\sigma_{c}\right)^{2}\right] .
$$

In order to prove Theorem 6.1, we need the following technical lemma.

Lemma 6.2. Consider $\dot{\gamma}$ a function of time that satisfies (3.1). Denote by $\gamma(t)=$ $\int_{0}^{t} \dot{\gamma}(s) d s$. Then, for all $t>\frac{2 \sigma_{c}}{m_{\dot{\gamma}}}$ and almost all $\sigma \in \mathbb{R}$, we have

$$
\int_{0}^{t} \chi(\sigma-\gamma(t)+\gamma(u)) d u \geq t-\frac{2 \sigma_{c}}{m_{\dot{\gamma}}}
$$

Proof. Denote by $Z(t, \sigma)=\int_{0}^{t} \chi(\sigma-\gamma(t)+\gamma(u)) d u$. For all $t>\frac{2 \sigma_{c}}{m_{\dot{\gamma}}}$ and almost 
all $\sigma \in \mathbb{R}$, we have

$$
\begin{aligned}
Z(t, & \sigma) \\
= & \int_{0}^{t}\left(\mathbb{1}_{\left(-\infty,-\sigma_{c}\right)}+\mathbb{1}_{\left(\sigma_{c}, \infty\right)}\right)(\sigma-\gamma(t)+\gamma(u)) d u \\
= & \int_{0}^{t}\left(\mathbb{1}_{\left(-\infty, \gamma^{-1}\left(-\sigma_{c}+\gamma(t)-\sigma\right)\right)}+\mathbb{1}_{\left(\gamma^{-1}\left(\sigma_{c}+\gamma(t)-\sigma\right), \infty\right)}\right)(u) d u \\
= & \begin{cases}t & \sigma<-\sigma_{c} \\
\gamma^{-1}\left(-\sigma_{c}+\gamma(t)-\sigma\right) & -\sigma_{c}<\sigma<\gamma(t)-\sigma_{c} \\
0 & \gamma(t)-\sigma_{c}<\sigma\end{cases} \\
& +\left\{\begin{array}{lll}
0 & \sigma<\sigma_{c} \\
t-\gamma^{-1}\left(\sigma_{c}+\gamma(t)-\sigma\right) & \sigma_{c}<\sigma<\gamma(t)+\sigma_{c} \\
t & \gamma(t)+\sigma_{c}<\sigma & -\sigma_{c}<\sigma \leq \sigma_{c} \\
= & \begin{cases}\gamma^{-1}\left(-\sigma_{c}+\gamma(t)-\sigma\right) & \\
t+\gamma^{-1}\left(-\sigma_{c}+\gamma(t)-\sigma\right)-\gamma^{-1}\left(\sigma_{c}+\gamma(t)-\sigma\right) & \sigma_{c}<\sigma \leq \gamma(t)+\sigma_{c} \\
t-\gamma^{-1}\left(\sigma_{c}+\gamma(t)-\sigma\right) & \text { or } \sigma>\gamma(t)+\sigma_{c} \\
t & \end{cases}
\end{array}\right.
\end{aligned}
$$

using that $\gamma(t)>2 \sigma_{c}$. We now estimate the above expression depending on $\sigma$. For almost all $\sigma \in\left(-\sigma_{c}, \sigma_{c}\right)$, the function $Z$ is decreasing in $\sigma$ so that $Z(t, \sigma) \geq \gamma^{-1}(\gamma(t)-$ $\left.2 \sigma_{c}\right)$. Moreover, because of (3.1), the function $\dot{\gamma}$ satisfies

$$
\gamma(t)-\gamma\left(t-\frac{2 \sigma_{c}}{m_{\dot{\gamma}}}\right)=\int_{0}^{t} \dot{\gamma}-\int_{0}^{t-\frac{2 \sigma_{c}}{m_{\dot{\gamma}}}} \dot{\gamma} \geq 2 \sigma_{c}
$$

so that $\gamma^{-1}\left(\gamma(t)-2 \sigma_{c}\right) \geq t-\frac{2 \sigma_{c}}{m_{\dot{\gamma}}}$, hence 6.6 for almost all $\sigma \in\left(-\sigma_{c}, \sigma_{c}\right)$.

Additionally, because of (3.1), the function $\gamma$ satisfies, for all $v>u \geq 0$,

$$
\gamma(v)-\gamma(u)=\int_{u}^{v} \dot{\gamma} \geq m_{\dot{\gamma}}(v-u) .
$$

This yields that $\gamma^{-1}$ is Lipschitz with a Lipschitz constant $\frac{1}{m_{\dot{\gamma}}}$ on $[0, \infty)$. Therefore, for almost $\sigma \in\left(\sigma_{c}, \gamma(t)-\sigma_{c}\right)$,

$$
t-Z(t, \sigma)=\gamma^{-1}\left(\sigma_{c}+\gamma(t)-\sigma\right)-\gamma^{-1}\left(-\sigma_{c}+\gamma(t)-\sigma\right) \leq \frac{2 \sigma_{c}}{m_{\dot{\gamma}}},
$$

hence (6.6).

For almost all $\sigma \in\left(\gamma(t)-\sigma_{c}, \gamma(t)+\sigma_{c}\right)$, the function $Z$ is increasing in $\sigma$ so that $Z(t, \sigma) \geq t-\gamma^{-1}\left(2 \sigma_{c}\right)$. Moreover,

$$
2 \sigma_{c}=\int_{0}^{\frac{2 \sigma_{c}}{m_{\dot{\gamma}}}} m_{\dot{\gamma}} \leq \int_{0}^{\frac{2 \sigma_{c}}{m_{\dot{\gamma}}}} \dot{\gamma}(u) d u=\gamma\left(\frac{2 \sigma_{c}}{m_{\dot{\gamma}}}\right)
$$

so that $\gamma^{-1}\left(2 \sigma_{c}\right) \leq \frac{2 \sigma_{c}}{m_{\dot{\gamma}}}$, hence 6.6 for almost all $\sigma \in\left(\gamma(t)-\sigma_{c}, \gamma(t)+\sigma_{c}\right)$.

The result (6.6) also holds in the case $\sigma \in \mathbb{R} \backslash\left[-\sigma_{c}, \gamma(t)+\sigma_{c}\right]$ where $Z(t, \sigma)=t$. This ends the proof. $\square$ 
Now that we have proved the technical Lemma 6.2, we turn to the

Proof of Theorem 6.1. The proof is divided into six steps. The first step establishes a delay differential equation on a function $g_{\epsilon}$, for which an explicit decomposition is known thanks to the Lemma 5.3. We then rewrite $g_{\epsilon}$ in a different form whose terms are estimated in Steps 3 and 4 . In the last two steps, we use these estimates to obtain (6.4) and then (6.5).

Before we get to the proof we introduce some notation. The scalars $\theta>0, \epsilon>0$ are fixed and satisfy $\frac{\theta}{\epsilon}>2 \frac{\sigma_{c}}{m_{\dot{\gamma}}}$. In Section 5 , we have introduced

$$
\omega_{\theta}=\frac{\sigma_{c}}{\dot{\gamma}(\theta)}
$$

which from bounds 6.1 on $\dot{\gamma}$ satisfies

$$
\frac{\sigma_{c}}{M_{\dot{\gamma}}}<\omega_{\theta}<\frac{\sigma_{c}}{m_{\dot{\gamma}}}
$$

We can therefore apply Lemma 5.4 to the function $k_{\theta}$ satisfying (5.11) with $\omega=\omega_{\theta}$, so that there exist $b, C_{0}>0$ which depend only on $\sigma_{c}, m_{\dot{\gamma}}$ and $M_{\dot{\gamma}}$ such that (5.16) and (5.17) hold for all $t>0$. Notably, $b$ and $C_{0}$ are independent from $\theta$ (and $\epsilon$ ).

Step 1: Applying Lemma 5.3. For a fixed $\theta$, denote by

$$
A_{\theta}(t)=\int \chi(\sigma) p_{0}(\sigma-\dot{\gamma}(\theta) t) \mathrm{e}^{-\frac{1}{\dot{\gamma}(\theta)} \int_{\sigma-\dot{\gamma}(\theta) t}^{\sigma} \chi(v) d v} d \sigma
$$

and by $\phi_{\theta}$ the solution to

$$
\dot{\phi}_{\theta}(t)+\phi_{\theta}(t)-\phi_{\theta}\left(t-\omega_{\theta}\right)=\dot{A}_{\theta}(t)+A_{\theta}(t)
$$

with the initial condition $\phi_{\theta}(t)=A_{\theta}(t), 0<t<\omega_{\theta}$. Consistently with (1.4), let us also introduce $f_{\epsilon}(t)=\int \chi(\sigma) p_{\epsilon}(t, \sigma) d \sigma$ where $p_{\epsilon}$ satisfies 6.2). Then

$$
g_{\epsilon}(t)=f_{\epsilon}(t)-\phi_{\theta}(t)
$$

belongs to $W^{1,1}(0, T)$ (because $f_{\epsilon}$ and $\phi_{\theta}$ do, see Lemma 4.4 and satisfies, for almost all $t>\omega_{\theta}$

$$
\dot{g}_{\epsilon}(t)+g_{\epsilon}(t)-g_{\epsilon}\left(t-\omega_{\theta}\right)=\dot{f}_{\epsilon}(t)+f_{\epsilon}(t)-\dot{A}_{\theta}(t)-A_{\theta}(t)-f_{\epsilon}\left(t-\omega_{\theta}\right) .
$$

Introduce $s>2 \frac{\sigma_{c}}{m_{\dot{\gamma}}}$. We apply Lemma 5.3 and obtain (using the same computations as in 5.28) above and the fact that $\left.f_{\epsilon}(0)=\phi_{\theta}(0)\right)$,

$$
\begin{aligned}
g_{\epsilon}(s)= & \left(f_{\epsilon}-\phi_{\theta}\right)\left(\omega_{\theta}\right) k_{\theta}\left(s-\omega_{\theta}\right)+\int_{0}^{\omega_{\theta}}\left(f_{\epsilon}-\phi_{\theta}\right)(t) k_{\theta}\left(s-t-\omega_{\theta}\right) d t \\
& +\int_{\omega_{\theta}}^{s}\left(\dot{f}_{\epsilon}(t)+f_{\epsilon}(t)-\dot{A}_{\theta}(t)-A_{\theta}(t)-f_{\epsilon}\left(t-\omega_{\theta}\right)\right) k_{\theta}(s-t) d t \\
(6.10) \quad & \int_{0}^{s}\left(\dot{f}_{\epsilon}(t)+f_{\epsilon}(t)-\dot{A}_{\theta}(t)-A_{\theta}(t)\right) k_{\theta}(s-t) d t-\int_{\omega_{\theta}}^{s} f_{\epsilon}\left(t-\omega_{\theta}\right) k_{\theta}(s-t) d t .
\end{aligned}
$$


Step 2: Rewriting $g_{\epsilon}$. In order to rewrite $g_{\epsilon}$ we show that, for almost all $t \in(0, s)$,

$$
\dot{f}_{\epsilon}(t)+f_{\epsilon}(t)=\dot{\gamma}(\epsilon t)\left(p_{\epsilon}\left(t, \sigma_{c}\right)-p_{\epsilon}\left(t,-\sigma_{c}\right)\right) .
$$

First, the function $p_{\epsilon}$ solution to 6.2 with $p_{0}$ as initial condition satisfies, for all $\eta \in \mathcal{D}([0, s) \times \mathbb{R})$,

$$
-\int_{0}^{s} \int_{\mathbb{R}} p_{\epsilon}\left(\frac{\partial \eta}{\partial t}+\dot{\gamma}(\epsilon t) \frac{\partial \eta}{\partial \sigma}-\chi \eta\right)=\int_{\mathbb{R}} p_{0}(\sigma) \eta(0, \sigma) d \sigma+\int_{0}^{s} f_{\epsilon}(t) \eta(t, 0) d t .
$$

Denote $\rho$ a function of $\mathcal{D}((0, s)), \rho^{n}$ a mollifier on $\mathbb{R}$ and $\chi^{n}=\rho^{n} * \chi$. Inserting $\eta^{n}(t, \sigma)=\chi^{n}(\sigma) \rho(t)$ in 6.12 yields, for $n$ sufficiently large such that $\chi^{n}(0)=0$,

$$
-\int_{0}^{s} \int_{\mathbb{R}} p_{\epsilon}\left(\frac{\partial \eta^{n}}{\partial t}+\dot{\gamma}(\epsilon t) \frac{\partial \eta^{n}}{\partial \sigma}-\chi \eta^{n}\right)=0
$$

which rewrites

$-\int_{0}^{s} \dot{\rho}(t) \int \chi^{n} p_{\epsilon}(t, \cdot) d t-\int \dot{\chi}^{n}(\sigma) \int_{0}^{s} \rho(t) \dot{\gamma}(\epsilon t) p_{\epsilon}(t, \sigma) d t d \sigma+\int_{0}^{s} \rho(t) \int \chi \chi^{n} p_{\epsilon}(t, \cdot) d t$

The function $t \mapsto\left(\sigma \mapsto p_{\epsilon}(t, \sigma)\right)$ belongs to $C\left([0, s], L^{1}\right)$, see Theorem 3.1, so that, by the dominated convergence theorem, for all $t \in[0, s], \int \chi^{n} p_{\epsilon}(t, \cdot)$ and $\int \chi \chi^{n} p_{\epsilon}(t, \cdot)$ converge to $f_{\epsilon}(t)$ defined by (1.4) as $n$ goes to infinity. Moreover $\sigma \mapsto\left(t \mapsto p_{\epsilon}(t, \sigma)\right)$ belongs to $C\left(\mathbb{R}, L^{1}(0, s)\right.$ ) (the proof is similar to the one in Step 2 of Theorem 3.1) so that $\int_{0}^{s} \rho(t) \dot{\gamma}(\epsilon t) p_{\epsilon}(t, \sigma) d t$ is continuous in $\sigma$. Passing to the limit $n \rightarrow \infty$ in the above equation yields

$$
-\int_{0}^{s} \dot{\rho}(t) f_{\epsilon}(t) d t-\int_{0}^{s} \rho(t) \dot{\gamma}(\epsilon t)\left(p_{\epsilon}\left(t, \sigma_{c}\right)-p_{\epsilon}\left(t,-\sigma_{c}\right)\right) d t+\int_{0}^{s} \rho(t) f_{\epsilon}(t) d t=0,
$$

hence 6.11 since $f_{\epsilon}$ and $p_{\epsilon}\left(\cdot, \pm \sigma_{c}\right)$ belong to $L^{1}(0, s)$.

We then denote

$$
Q_{\epsilon}(t)=\dot{\gamma}(\epsilon t)\left(p_{\epsilon}\left(t, \sigma_{c}\right)-p_{\epsilon}\left(t,-\sigma_{c}\right)\right)-\dot{A}_{\theta}(t)-A_{\theta}(t)-f_{\epsilon}\left(t-\omega_{\theta}\right) \mathbb{1}_{\left(\omega_{\theta}, s\right)}(t)
$$

so that the expression 6.10 on $g_{\epsilon}$ rewrites

$$
\begin{aligned}
g_{\epsilon}(s) & =\int_{0}^{s} Q_{\epsilon}(t) k_{\theta}(s-t) d t \\
& =\frac{1}{1+\omega_{\theta}} \int_{0}^{s} Q_{\epsilon}(t) d t+\int_{0}^{s} Q_{\epsilon}(t) k_{\theta, 1}(s-t) d t
\end{aligned}
$$

using the decomposition $k_{\theta}=\frac{1}{1+\omega_{\theta}}+k_{1, \theta}$ (see 5.16$)$ ) that was established in Lemma 5.4 . We now derive estimates on the two terms of the above expression, when $s=\frac{\theta}{\epsilon}$.

Step 3: Estimate of $\int_{0}^{\frac{\theta}{\epsilon}} Q_{\epsilon}$. Introduce

$$
\eta_{-}(t, \sigma)=\mathbb{1}_{\left[-\sigma_{c}-\dot{\gamma}(\theta)(s-t),-\sigma_{c}\right]}(\sigma) \mathrm{e}^{\frac{\sigma+\sigma_{c}}{\dot{\gamma}(\theta)}}
$$

which satisfies, in $\mathcal{D}^{\prime}([0, s) \times \mathbb{R})$,

$$
\frac{\partial \eta_{-}}{\partial \sigma}=-\delta_{-\sigma_{c}}(\sigma)+\delta_{-\sigma_{c}-\dot{\gamma}(\theta)(s-t)}(\sigma) \mathrm{e}^{t-s}+\frac{1}{\dot{\gamma}(\theta)} \mathbb{1}_{\left[-\sigma_{c}-\dot{\gamma}(\theta)(s-t),-\sigma_{c}\right]}(\sigma) \mathrm{e}^{\frac{\sigma+\sigma_{c}}{\dot{\gamma}(\theta)}}
$$


and

$$
\begin{aligned}
-\frac{\partial \eta_{-}}{\partial t}-\dot{\gamma}(\epsilon t) \frac{\partial \eta_{-}}{\partial \sigma}+\chi \eta_{-}= & \dot{\gamma}(\epsilon t) \delta_{-\sigma_{c}}(\sigma)+(\dot{\gamma}(\theta)-\dot{\gamma}(\epsilon t)) \delta_{-\sigma_{c}-\dot{\gamma}(\theta)(s-t)}(\sigma) \mathrm{e}^{t-s} \\
& +\frac{\dot{\gamma}(\theta)-\dot{\gamma}(\epsilon t)}{\dot{\gamma}(\theta)} \mathbb{1}_{\left[-\sigma_{c}-\dot{\gamma}(\theta)(s-t),-\sigma_{c}\right]}(\sigma) \mathrm{e}^{\frac{\sigma+\sigma_{c}}{\dot{\gamma}(\theta)}} .
\end{aligned}
$$

For $n, m \in \mathbb{N}$, take as a test function

$$
\eta=\eta_{-}^{n, m}(t, \sigma)=\rho^{n} * \mathbb{1}_{\left[-\sigma_{c}-\dot{\gamma}(\theta)(s-t),-\sigma_{c}\right]}(\sigma) \mathrm{e}^{\frac{\sigma+\sigma_{c}}{\dot{\gamma}(\theta)}} \zeta_{[0, s)}^{m}(t)
$$

in 6.12 and pass to the limit in $n$ and then $m$. Here and in the following, $\zeta_{[0, s)}^{m}$ denotes a $C^{\infty}([0, s), \mathbb{R})$ function with compact support in $[0, s)$, such that $\zeta_{[0, s)}^{m}$ converges pointwise to $\mathbb{1}_{[0, s)}$.

We omit the details, the arguments being similar to those in 6.13 . We obtain

$$
\begin{aligned}
\int_{0}^{s} \dot{\gamma}(\epsilon t) p_{\epsilon}\left(t,-\sigma_{c}\right) d t & +\int_{0}^{s}(\dot{\gamma}(\theta)-\dot{\gamma}(\epsilon t)) p_{\epsilon}\left(t,-\sigma_{c}-\dot{\gamma}(\theta)(s-t)\right) \mathrm{e}^{t-s} d t \\
& +\int_{0}^{s} \int_{-\sigma_{c}-\dot{\gamma}(\theta)(s-t)}^{-\sigma_{c}} \frac{\dot{\gamma}(\theta)-\dot{\gamma}(\epsilon t)}{\dot{\gamma}(\theta)} p_{\epsilon}(t, \sigma) \mathrm{e}^{\frac{\sigma+\sigma_{c}}{\dot{\gamma}(\theta)}} d \sigma d t \\
& =\int_{-\sigma_{c}-\dot{\gamma}(\theta) s}^{-\sigma_{c}} p_{0}(\sigma) \mathrm{e}^{\frac{\sigma+\sigma_{c}}{\dot{\gamma}(\theta)}} d \sigma .
\end{aligned}
$$

With changes of variable $\sigma=-\sigma_{c}-\dot{\gamma}(\theta)(u-t)$ and $\sigma=-\sigma_{c}-\dot{\gamma}(\theta) t$ in the last two integrals, this rewrites

$$
\begin{array}{r}
\int_{0}^{s} \dot{\gamma}(\epsilon t) p_{\epsilon}\left(t,-\sigma_{c}\right) d t+\int_{0}^{s}(\dot{\gamma}(\theta)-\dot{\gamma}(\epsilon t)) p_{\epsilon}\left(t,-\sigma_{c}-\dot{\gamma}(\theta)(s-t)\right) \mathrm{e}^{t-s} d t \\
+\int_{0}^{s} \int_{t}^{s}(\dot{\gamma}(\theta)-\dot{\gamma}(\epsilon t)) p_{\epsilon}\left(t,-\sigma_{c}-\dot{\gamma}(\theta)(u-t)\right) \mathrm{e}^{t-u} d u d t \\
=\dot{\gamma}(\theta) \int_{0}^{s} p_{0}\left(-\sigma_{c}-\dot{\gamma}(\theta) t\right) \mathrm{e}^{-t} d t .
\end{array}
$$

Let us assume that $s>2 \omega_{\theta}$ and introduce

$$
\begin{aligned}
\eta_{+}(t, \sigma)= & -\mathbb{1}_{\left[\sigma_{c}-\dot{\gamma}(\theta)(s-t),-\sigma_{c}\right]}(\sigma) \mathrm{e}^{\frac{\sigma+\sigma_{c}}{\dot{\gamma}(\theta)}} \mathbb{1}_{\left(0, s-2 \omega_{\theta}\right)}(t) \\
& +\mathbb{1}_{\left[-\sigma_{c}, \sigma_{c}-\dot{\gamma}(\theta)(s-t)\right]}(\sigma) \mathbb{1}_{\left(s-2 \omega_{\theta}, s\right)}(t)-\mathbb{1}_{\left[-\sigma_{c}, \sigma_{c}\right]}(\sigma)
\end{aligned}
$$

which satisfies, in $\mathcal{D}^{\prime}((0, s) \times \mathbb{R})$,

$$
\begin{aligned}
\frac{\partial \eta_{+}}{\partial \sigma}= & \delta_{\sigma_{c}}-\delta_{\sigma_{c}-\dot{\gamma}(\theta)(s-t)}(\sigma) \mathrm{e}^{\frac{\sigma_{c}}{\dot{\gamma}(\theta)}+t-s} \mathbb{1}_{\left(0, s-2 \omega_{\theta}\right)}(t) \\
& -\frac{1}{\dot{\gamma}(\theta)} \mathrm{e}^{\frac{\sigma+\sigma_{c}}{\dot{\gamma}(\theta)}} \mathbb{1}_{\left[\sigma_{c}-\dot{\gamma}(\theta)(s-t),-\sigma_{c}\right]}(\sigma) \mathbb{1}_{\left(0, s-2 \omega_{\theta}\right)}(t)-\delta_{\sigma_{c}-\dot{\gamma}(\theta)(s-t)}(\sigma) \mathbb{1}_{\left(s-2 \omega_{\theta}, s\right)}(t)
\end{aligned}
$$

and

$$
\begin{aligned}
- & \frac{\partial \eta_{+}}{\partial t}-\dot{\gamma}(\epsilon t) \frac{\partial \eta_{+}}{\partial \sigma}+\chi \eta_{+} \\
= & -\dot{\gamma}(\epsilon t) \delta_{\sigma_{c}}-(\dot{\gamma}(\theta)-\dot{\gamma}(\epsilon t)) \delta_{\sigma_{c}-\dot{\gamma}(\theta)(s-t)}(\sigma) \mathrm{e}^{2 \frac{\sigma_{c}}{\dot{\gamma}(\theta)}+t-s} \mathbb{1}_{\left(0, s-2 \omega_{\theta}\right)}(t) \\
& \quad-\frac{\dot{\gamma}(\theta)-\dot{\gamma}(\epsilon t)}{\dot{\gamma}(\theta)} \mathrm{e}^{\frac{\sigma+\sigma_{c}}{\dot{\gamma}(\theta)}} \mathbb{1}_{\left[\sigma_{c}-\dot{\gamma}(\theta)(s-t),-\sigma_{c}\right]}(\sigma) \mathbb{1}_{\left(0, s-2 \omega_{\theta}\right)}(t) \\
& \quad-(\dot{\gamma}(\theta)-\dot{\gamma}(\epsilon t)) \delta_{\sigma_{c}-\dot{\gamma}(\theta)(s-t)}(\sigma) \mathbb{1}_{\left(s-2 \omega_{\theta}, s\right)}(t) .
\end{aligned}
$$


We again use a regularization

$$
\begin{aligned}
\eta_{+}^{n, m}= & -\rho^{n} * \mathbb{1}_{\left[\sigma_{c}-\dot{\gamma}(\theta)(s-t),-\sigma_{c}\right]}(\sigma) \mathrm{e}^{\frac{\sigma+\sigma_{c}}{\dot{\gamma}(\theta)}} \zeta_{\left[0, s-2 \omega_{\theta}\right)}^{m}(t) \\
& +\rho^{n} * \mathbb{1}_{\left[-\sigma_{c}, \sigma_{c}-\dot{\gamma}(\theta)(s-t)\right]}(\sigma) \zeta_{\left(s-2 \omega_{\theta}, s\right)}^{m}(t) \\
& -\rho^{n} * \mathbb{1}_{\left[-\sigma_{c}, \sigma_{c}\right]}(\sigma) \zeta_{[0, s)}^{m}(t)
\end{aligned}
$$

and pass to the limit in 6.12

$$
\begin{array}{r}
-\int_{0}^{s} \dot{\gamma}(\epsilon t) p_{\epsilon}\left(t, \sigma_{c}\right) d t-\int_{0}^{s-2 \omega_{\theta}}(\dot{\gamma}(\theta)-\dot{\gamma}(\epsilon t)) p_{\epsilon}\left(t, \sigma_{c}-\dot{\gamma}(\theta)(s-t)\right) \mathrm{e}^{\frac{2 \sigma_{c}}{\dot{\gamma}(\theta)}+t-s} d t \\
-\int_{0}^{s-2 \omega_{\theta}} \int_{t+2 \omega_{\theta}}^{s}(\dot{\gamma}(\theta)-\dot{\gamma}(\epsilon t)) p_{\epsilon}\left(t, \sigma_{c}-\dot{\gamma}(\theta)(u-t)\right) \mathrm{e}^{\frac{2 \sigma_{c}}{\dot{\gamma}(\theta)}+t-u} d u d t \\
-\int_{s-2 \omega_{\theta}}^{s}(\dot{\gamma}(\theta)-\dot{\gamma}(\epsilon t)) p_{\epsilon}\left(t, \sigma_{c}-\dot{\gamma}(\theta)(s-t)\right) d t \\
=-\dot{\gamma}(\theta) \int_{0}^{s} p_{0}\left(\sigma_{c}-\dot{\gamma}(\theta) t\right) \mathrm{e}^{-\frac{1}{\dot{\gamma}(\theta)} \int_{\sigma_{c}-\dot{\gamma}(\theta) t}^{\sigma_{c}} \chi} d t-\int_{0}^{s-\frac{\sigma_{c}}{\dot{\gamma}(\theta)}} f_{\epsilon} .
\end{array}
$$

In addition, from its definition (6.8), we know that $A_{\theta}$ satisfies (see (4.5)

$$
\begin{aligned}
\int_{0}^{s} \dot{A}_{\theta}+A_{\theta}= & -\dot{\gamma}(\theta) \int_{0}^{s} p_{0}\left(-\sigma_{c}-\dot{\gamma}(\theta) t\right) \mathrm{e}^{-t} d t \\
& +\dot{\gamma}(\theta) \int_{0}^{s} p_{0}\left(\sigma_{c}-\dot{\gamma}(\theta) t\right) \mathrm{e}^{-\frac{1}{\dot{\gamma}(\theta)} \int_{\sigma_{c}-\dot{\gamma}(\theta) t}^{\sigma_{c}} \chi} d t .
\end{aligned}
$$

Summing up expressions 6.18, 6.20 and 6.21, we obtain

$$
\begin{aligned}
\int_{0}^{s} Q_{\epsilon}= & \int_{0}^{s}(\dot{\gamma}(\theta)-\dot{\gamma}(\epsilon t)) p_{\epsilon}\left(t,-\sigma_{c}-\dot{\gamma}(\theta)(s-t)\right) \mathrm{e}^{t-s} d t \\
& +\int_{0}^{s} \int_{t}^{s}(\dot{\gamma}(\theta)-\dot{\gamma}(\epsilon t)) p_{\epsilon}\left(t,-\sigma_{c}-\dot{\gamma}(\theta)(u-t)\right) \mathrm{e}^{t-u} d u d t \\
& -\int_{0}^{s-2 \omega_{\theta}} \int_{t+2 \omega_{\theta}}^{s}(\dot{\gamma}(\theta)-\dot{\gamma}(\epsilon t)) p_{\epsilon}\left(t, \sigma_{c}-\dot{\gamma}(\theta)(v-t)\right) \mathrm{e}^{2 \omega_{\theta}+t-v} d v d t \\
& -\int_{0}^{s-2 \omega_{\theta}}(\dot{\gamma}(\theta)-\dot{\gamma}(\epsilon t)) p_{\epsilon}\left(t, \sigma_{c}-\dot{\gamma}(\theta)(s-t)\right) \mathrm{e}^{2 \omega_{\theta}+t-s} d t \\
& -\int_{s-2 \omega_{\theta}}^{s}(\dot{\gamma}(\theta)-\dot{\gamma}(\epsilon t)) p_{\epsilon}\left(t, \sigma_{c}-\dot{\gamma}(\theta)(s-t)\right) d t .
\end{aligned}
$$

Taking $s=\frac{\theta}{\epsilon}$ and summing up the second and the third term (with the change of 
variable $u=v-2 \omega_{\theta}$ in the third term), this rewrites

$$
\begin{aligned}
\int_{0}^{\frac{\theta}{\epsilon}} Q_{\epsilon}= & \int_{0}^{\frac{\theta}{\epsilon}}(\dot{\gamma}(\theta)-\dot{\gamma}(\epsilon t)) p_{\epsilon}\left(t,-\sigma_{c}-\dot{\gamma}(\theta)\left(\frac{\theta}{\epsilon}-t\right)\right) \mathrm{e}^{t-\frac{\theta}{\epsilon}} d t \\
& +\int_{\frac{\theta}{\epsilon}-2 \omega_{\theta}}^{\frac{\theta}{\epsilon}} \int_{t}^{\frac{\theta}{\epsilon}}(\dot{\gamma}(\theta)-\dot{\gamma}(\epsilon t)) p_{\epsilon}\left(t,-\sigma_{c}-\dot{\gamma}(\theta)(u-t)\right) \mathrm{e}^{t-u} d u d t \\
& +\int_{0}^{\frac{\theta}{\epsilon}-2 \omega_{\theta}} \int_{\frac{\theta}{\epsilon}-2 \omega_{\theta}}^{\frac{\theta}{\epsilon}}(\dot{\gamma}(\theta)-\dot{\gamma}(\epsilon t)) p_{\epsilon}\left(t,-\sigma_{c}-\dot{\gamma}(\theta)(u-t)\right) \mathrm{e}^{t-u} d u d t \\
& -\int_{0}^{\frac{\theta}{\epsilon}-2 \omega_{\theta}}(\dot{\gamma}(\theta)-\dot{\gamma}(\epsilon t)) p_{\epsilon}\left(t, \sigma_{c}-\dot{\gamma}(\theta)\left(\frac{\theta}{\epsilon}-t\right)\right) \mathrm{e}^{2 \omega_{\theta}+t-\frac{\theta}{\epsilon}} d t \\
& -\int_{\frac{\theta}{\epsilon}-2 \omega_{\theta}}^{\frac{\theta}{\epsilon}}(\dot{\gamma}(\theta)-\dot{\gamma}(\epsilon t)) p_{\epsilon}\left(t, \sigma_{c}-\dot{\gamma}(\theta)\left(\frac{\theta}{\epsilon}-t\right)\right) d t .
\end{aligned}
$$

Using the Lipschitz property of $\dot{\gamma}$ and $L^{\infty}$-bound 4.3 on $p_{\epsilon}$, this implies

$$
\begin{aligned}
\left|\int_{0}^{\frac{\theta}{\epsilon}} Q_{\epsilon}\right| \leq & C_{\infty} L_{\dot{\gamma}} \int_{0}^{\frac{\theta}{\epsilon}}(\theta-\epsilon t) \mathrm{e}^{t-\frac{\theta}{\epsilon}} d t \\
& +C_{\infty} L_{\dot{\gamma}} \int_{\frac{\theta}{\epsilon}-2 \omega_{\theta}}^{\frac{\theta}{\epsilon}} \int_{t}^{\frac{\theta}{\epsilon}}(\theta-\epsilon t) \mathrm{e}^{t-u} d u d t \\
& +C_{\infty} L_{\dot{\gamma}} \int_{0}^{\frac{\theta}{\epsilon}-2 \omega_{\theta}} \int_{\frac{\theta}{\epsilon}-2 \omega_{\theta}}^{\frac{\theta}{\epsilon}}(\theta-\epsilon t) \mathrm{e}^{t-u} d u d t \\
& +C_{\infty} L_{\dot{\gamma}} \int_{0}^{\frac{\theta}{\epsilon}-2 \omega_{\theta}}(\theta-\epsilon t) \mathrm{e}^{2 \omega_{\theta}+t-\frac{\theta}{\epsilon}} d t \\
& +C_{\infty} L_{\dot{\gamma}} \int_{\frac{\theta}{\epsilon}-2 \omega_{\theta}}^{\frac{\theta}{\epsilon}}(\theta-\epsilon t) d t .
\end{aligned}
$$

For a constant $\alpha<0$, we have

$$
\begin{aligned}
\int_{0}^{\frac{\theta}{\epsilon}}(\theta-\epsilon v) \mathrm{e}^{\alpha\left(\frac{\theta}{\epsilon}-v\right)} d v & =\frac{\epsilon}{\alpha^{2}} \int_{0}^{-\frac{\alpha \theta}{\epsilon}} u \mathrm{e}^{-u} d u \\
& <\frac{\epsilon}{\alpha^{2}} \int_{0}^{\infty} u \mathrm{e}^{-u} d u .
\end{aligned}
$$

Using estimate 6.23 or variants, we find

$$
\begin{aligned}
\left|\int_{0}^{\frac{\theta}{\epsilon}} Q_{\epsilon}\right| \leq C_{\infty} L_{\dot{\gamma}} \epsilon & \left(\int_{0}^{\infty} u \mathrm{e}^{-u} d u+4 \omega_{\theta}^{2}+\left(1+\mathrm{e}^{2 \omega_{\theta}}\right) \int_{0}^{\infty} u \mathrm{e}^{-u} d u\right. \\
& \left.+\mathrm{e}^{2 \omega_{\theta}} \int_{0}^{\infty} u \mathrm{e}^{-u} d u+2 \omega_{\theta}^{2}\right) \\
\leq & C_{\infty} L_{\dot{\gamma}} \epsilon\left(2+6 \omega_{\theta}^{2}+2 \mathrm{e}^{2 \omega_{\theta}}\right) .
\end{aligned}
$$

Since $\omega_{\theta}<\frac{\sigma_{c}}{m_{\dot{\gamma}}}$, we obtain

$$
\left|\int_{0}^{\frac{\theta}{\epsilon}} Q_{\epsilon}\right| \leq K C_{\infty} L_{\dot{\gamma}} \epsilon
$$


with $K$ a constant that is independent from $\theta$ and $\epsilon$. Throughout the rest of the proof below, we will likewise denote by $K$ such a constant, whose precise value may change from one occurrence to another.

Step 4: Estimate of $\int_{0}^{\frac{\theta}{\epsilon}} Q_{\epsilon}(t) k_{\theta, 1}\left(\frac{\theta}{\epsilon}-t\right) d t$. Inserting expression 6.8) of $A_{\theta}$ (see 6.21) above for a similar computation), $Q_{\epsilon}$ defined by (6.14) satisfies

$$
\begin{aligned}
& \int_{0}^{\frac{\theta}{\epsilon}} Q_{\epsilon}(t) k_{\theta, 1}\left(\frac{\theta}{\epsilon}-t\right) d t=\int_{0}^{\frac{\theta}{\epsilon}} \dot{\gamma}(\epsilon t)\left(p_{\epsilon}\left(t, \sigma_{c}\right)-p_{\epsilon}\left(t,-\sigma_{c}\right)\right) k_{\theta, 1}\left(\frac{\theta}{\epsilon}-t\right) d t \\
& \quad+\dot{\gamma}(\theta) \int_{0}^{\frac{\theta}{\epsilon}}\left(p_{0}\left(-\sigma_{c}-\dot{\gamma}(\theta) t\right) \mathrm{e}^{-t}-p_{0}\left(\sigma_{c}-\dot{\gamma}(\theta) t\right) \mathrm{e}^{-\frac{1}{\dot{\gamma}(\theta)} \int_{\sigma_{c}-\dot{\gamma}(\theta) t}^{\sigma_{c}} \chi}\right) k_{\theta, 1}\left(\frac{\theta}{\epsilon}-t\right) d t \\
& \quad-\int_{\omega_{\theta}}^{\frac{\theta}{\epsilon}} f_{\epsilon}\left(t-\omega_{\theta}\right) k_{\theta, 1}\left(\frac{\theta}{\epsilon}-t\right) d t .
\end{aligned}
$$

For further use, notice that the integral in the second line above can be rewritten as:

$$
\begin{aligned}
& \int_{0}^{\frac{\theta}{\epsilon}}\left(p_{0}\left(-\sigma_{c}-\dot{\gamma}(\theta) t\right) \mathrm{e}^{-t}-p_{0}\left(\sigma_{c}-\dot{\gamma}(\theta) t\right) \mathrm{e}^{-\frac{1}{\dot{\gamma}(\theta)} \int_{\sigma_{c}-\dot{\gamma}(\theta) t}^{\sigma_{c}} \chi}\right) k_{\theta, 1}\left(\frac{\theta}{\epsilon}-t\right) d t \\
& =\int_{-\sigma_{c}-\dot{\gamma}(\theta) \frac{\theta}{\epsilon}}^{-\sigma_{c}} p_{0}(\sigma) \mathrm{e}^{\frac{\sigma+\sigma_{c}}{\dot{\gamma}(\theta)}} k_{\theta, 1}\left(\frac{\theta}{\epsilon}+\frac{\sigma+\sigma_{c}}{\dot{\gamma}(\theta)}\right) d t \\
& \quad-\int_{\sigma_{c}-\dot{\gamma}(\theta) \frac{\theta}{\epsilon}}^{\sigma_{c}} p_{0}(\sigma) \mathrm{e}^{-\frac{1}{\dot{\gamma}(\theta)} \int_{\sigma}^{\sigma_{c}} \chi} k_{\theta, 1}\left(\frac{\theta}{\epsilon}+\frac{\sigma-\sigma_{c}}{\dot{\gamma}(\theta)}\right) d t .
\end{aligned}
$$

In order to rewrite the first term of the right-hand side, we use again the functions $\eta_{-}$and $\eta_{+}$respectively defined by 6.16) and (6.19). Using a regularization of $\eta_{-}(t, \sigma) k_{\theta, 1}\left(\frac{\theta}{\epsilon}-t+\frac{\sigma+\sigma_{c}}{\dot{\gamma}(\theta)}\right)$ as test function in 6.12) (with $\left.s=\theta / \epsilon\right)$ and then passing to the limit, we obtain

$$
\begin{array}{r}
\int_{0}^{\frac{\theta}{\epsilon}} \int_{\mathbb{R}}\left(-\frac{\partial \eta_{-}}{\partial t}-\dot{\gamma}(\epsilon t) \frac{\partial \eta_{-}}{\partial \sigma}+\chi \eta_{-}\right)(t, \sigma) k_{\theta, 1}\left(\frac{\theta}{\epsilon}-t+\frac{\sigma+\sigma_{c}}{\dot{\gamma}(\theta)}\right) p_{\epsilon}(t, \sigma) d t d \sigma \\
+\int_{0}^{\frac{\theta}{\epsilon}} \int_{\mathbb{R}} \frac{\dot{\gamma}(\theta)-\dot{\gamma}(\epsilon t)}{\dot{\gamma}(\theta)} \eta_{-}(t, \sigma) \dot{k}_{\theta, 1}\left(\frac{\theta}{\epsilon}-t+\frac{\sigma+\sigma_{c}}{\dot{\gamma}(\theta)}\right) p_{\epsilon}(t, \sigma) d t d \sigma \\
=\int_{\mathbb{R}} p_{0}(\sigma) \eta_{-}(0, \sigma) k_{\theta, 1}\left(\frac{\theta}{\epsilon}+\frac{\sigma+\sigma_{c}}{\dot{\gamma}(\theta)}\right) d \sigma
\end{array}
$$

This rewrites (using similar computations as in 6.17) above)

$$
\begin{array}{r}
\int_{0}^{\frac{\theta}{\epsilon}} \dot{\gamma}(\epsilon t) p_{\epsilon}\left(t,-\sigma_{c}\right) k_{\theta, 1}\left(\frac{\theta}{\epsilon}-t\right) d t \\
+k_{\theta, 1}(0) \int_{0}^{\frac{\theta}{\epsilon}}(\dot{\gamma}(\theta)-\dot{\gamma}(\epsilon t)) p_{\epsilon}\left(t,-\sigma_{c}-\dot{\gamma}(\theta)\left(\frac{\theta}{\epsilon}-t\right)\right) \mathrm{e}^{t-\frac{\theta}{\epsilon}} d t \\
+\int_{0}^{\frac{\theta}{\epsilon}} \int_{-\sigma_{c}-\dot{\gamma}(\theta)\left(\frac{\theta}{\epsilon}-t\right)}^{-\sigma_{c}} \frac{\dot{\gamma}(\theta)-\dot{\gamma}(\epsilon t)}{\dot{\gamma}(\theta)} p_{\epsilon}(t, \sigma) \mathrm{e}^{\frac{\sigma+\sigma_{c}}{\dot{\gamma}(\theta)}}\left(k_{\theta, 1}+\dot{k}_{\theta, 1}\right)\left(\frac{\theta}{\epsilon}-t+\frac{\sigma+\sigma_{c}}{\dot{\gamma}(\theta)}\right) d \sigma d t \\
=\int_{-\sigma_{c}-\dot{\gamma}(\theta) \frac{\theta}{\epsilon}}^{-\sigma_{c}} p_{0}(\sigma) \mathrm{e}^{\frac{\sigma+\sigma_{c}}{\dot{\gamma}(\theta)}} k_{\theta, 1}\left(\frac{\theta}{\epsilon}+\frac{\sigma+\sigma_{c}}{\dot{\gamma}(\theta)}\right) d \sigma .
\end{array}
$$


Similarly, using a regularization of $\eta_{+}(t, \sigma) k_{\theta, 1}\left(\frac{\theta}{\epsilon}-t+\frac{\sigma-\sigma_{c}}{\dot{\gamma}(\theta)}\right)$ as test function in 6.12, we obtain

$$
\begin{array}{r}
\int_{0}^{\frac{\theta}{\epsilon}} \int_{\mathbb{R}}\left(-\frac{\partial \eta_{+}}{\partial t}-\dot{\gamma}(\epsilon t) \frac{\partial \eta_{+}}{\partial \sigma}+\chi \eta_{+}\right)(t, \sigma) k_{\theta, 1}\left(\frac{\theta}{\epsilon}-t+\frac{\sigma-\sigma_{c}}{\dot{\gamma}(\theta)}\right) p_{\epsilon}(t, \sigma) d t d \sigma \\
+\int_{0}^{\frac{\theta}{\epsilon}} \int_{\mathbb{R}} \frac{\dot{\gamma}(\theta)-\dot{\gamma}(\epsilon t)}{\dot{\gamma}(\theta)} \eta_{+}(t, \sigma) \dot{k}_{\theta, 1}\left(\frac{\theta}{\epsilon}-t+\frac{\sigma-\sigma_{c}}{\dot{\gamma}(\theta)}\right) p_{\epsilon}(t, \sigma) d t d \sigma \\
=\int_{\mathbb{R}} p_{0}(\sigma) \eta_{+}(0, \sigma) k_{\theta, 1}\left(\frac{\theta}{\epsilon}+\frac{\sigma-\sigma_{c}}{\dot{\gamma}(\theta)}\right) d \sigma+\int_{0}^{\frac{\theta}{\epsilon}} f_{\epsilon}(t) \eta_{+}(t, 0) k_{\theta, 1}\left(\frac{\theta}{\epsilon}-t-\frac{\sigma_{c}}{\dot{\gamma}(\theta)}\right) d t,
\end{array}
$$

so that (using similar computations as in 6.20 above)

$$
\begin{array}{r}
-\int_{0}^{\frac{\theta}{\epsilon}} \dot{\gamma}(\epsilon t) p_{\epsilon}\left(t, \sigma_{c}\right) k_{\theta, 1}\left(\frac{\theta}{\epsilon}-t\right) d t \\
-k_{\theta, 1}(0) \int_{0}^{\frac{\theta}{\epsilon}-2 \omega_{\theta}}(\dot{\gamma}(\theta)-\dot{\gamma}(\epsilon t)) p_{\epsilon}\left(t, \sigma_{c}-\dot{\gamma}(\theta)\left(\frac{\theta}{\epsilon}-t\right)\right) \mathrm{e}^{2 \omega_{\theta}+t-\frac{\theta}{\epsilon}} d t \\
-k_{\theta, 1}(0) \int_{\frac{\theta}{\epsilon}-2 \omega_{\theta}}^{\frac{\theta}{\epsilon}}(\dot{\gamma}(\theta)-\dot{\gamma}(\epsilon t)) p_{\epsilon}\left(t, \sigma_{c}-\dot{\gamma}(\theta)\left(\frac{\theta}{\epsilon}-t\right)\right) d t \\
-\int_{0}^{\frac{\theta}{\epsilon}-2 \omega_{\theta}} \int_{\sigma_{c}-\dot{\gamma}(\theta)\left(\frac{\theta}{\epsilon}-t\right)}^{-\sigma_{c}} \frac{\dot{\gamma}(\theta)-\dot{\gamma}(\epsilon t)}{\dot{\gamma}(\theta)} p_{\epsilon}(t, \sigma) \mathrm{e}^{\frac{\sigma+\sigma_{c}}{\dot{\gamma}(\theta)}}\left(k_{\theta, 1}+\dot{k}_{\theta, 1}\right)\left(\frac{\theta}{\epsilon}-t+\frac{\sigma-\sigma_{c}}{\dot{\gamma}(\theta)}\right) d \sigma d t \\
-\int_{\frac{\theta}{\epsilon}-2 \omega_{\theta}}^{\frac{\theta}{\epsilon}} \int_{\sigma_{c}-\dot{\gamma}(\theta)\left(\frac{\theta}{\epsilon}-t\right)}^{-\sigma_{c}} \frac{\dot{\gamma}(\theta)-\dot{\gamma}(\epsilon t)}{\dot{\gamma}(\theta)} p_{\epsilon}(t, \sigma) \dot{k}_{\theta, 1}\left(\frac{\theta}{\epsilon}-t+\frac{\sigma-\sigma_{c}}{\dot{\gamma}(\theta)}\right) d \sigma d t \\
-\int_{0}^{\frac{\theta}{\epsilon}} \int_{-\sigma_{c}}^{\sigma_{c}} \frac{\dot{\gamma}(\theta)-\dot{\gamma}(\epsilon t)}{\dot{\gamma}(\theta)} p_{\epsilon}(t, \sigma) \dot{k}_{\theta, 1}\left(\frac{\theta}{\epsilon}-t+\frac{\sigma-\sigma_{c}}{\dot{\gamma}(\theta)}\right) d \sigma d t \\
=-\int_{\sigma_{c}-\dot{\gamma}(\theta) \frac{\theta}{\epsilon}}^{-\sigma_{c}} p_{0}(\sigma) \mathrm{e}^{\frac{\sigma+\sigma_{c}}{\dot{\gamma}(\theta)}} k_{\theta, 1}\left(\frac{\theta}{\epsilon}+\frac{\sigma-\sigma_{c}}{\dot{\gamma}(\theta)}\right) d \sigma-\int_{-\sigma_{c}}^{\sigma_{c}} p_{0}(\sigma) k_{\theta, 1}\left(\frac{\theta}{\epsilon}+\frac{\sigma-\sigma_{c}}{\dot{\gamma}(\theta)}\right) d \sigma \\
(6.28) \quad-\int_{0}^{\frac{\theta}{\epsilon}-\omega_{\theta}} f_{\epsilon}(t) k_{\theta, 1}\left(\frac{\theta}{\epsilon}-t-\omega_{\theta}\right) d t .
\end{array}
$$

We now perform the linear combinations: 6.25$)-(\sqrt{6.27})+(6.28))$. The last two term of the right-hand side of 6.25 cancel out with the right-hand sides of 6.27) 
and (6.28) (using in particular 6.26) so that

$$
\begin{array}{r}
\int_{0}^{\frac{\theta}{\epsilon}} Q_{\epsilon}(t) k_{\theta, 1}\left(\frac{\theta}{\epsilon}-t\right) d t=k_{\theta, 1}(0) \int_{0}^{\frac{\theta}{\epsilon}}(\dot{\gamma}(\theta)-\dot{\gamma}(\epsilon t)) p_{\epsilon}\left(t,-\sigma_{c}-\dot{\gamma}(\theta)\left(\frac{\theta}{\epsilon}-t\right)\right) \mathrm{e}^{t-\frac{\theta}{\epsilon}} d t \\
+\int_{0}^{\frac{\theta}{\epsilon}} \int_{-\sigma_{c}-\dot{\gamma}(\theta)\left(\frac{\theta}{\epsilon}-t\right)}^{\left.-\sigma_{c}\right)} \frac{\dot{\gamma}(\theta)-\dot{\gamma}(\epsilon t)}{\dot{\gamma}(\theta)} p_{\epsilon}(t, \sigma) \mathrm{e}^{\frac{\sigma+\sigma_{c}}{\dot{\gamma}(\theta)}}\left(k_{\theta, 1}+\dot{k}_{\theta, 1}\right)\left(\frac{\theta}{\epsilon}-t+\frac{\sigma+\sigma_{c}}{\dot{\gamma}(\theta)}\right) d \sigma d t \\
-k_{\theta, 1}(0) \int_{0}^{\frac{\theta}{\epsilon}-2 \omega_{\theta}}(\dot{\gamma}(\theta)-\dot{\gamma}(\epsilon t)) p_{\epsilon}\left(t, \sigma_{c}-\dot{\gamma}(\theta)\left(\frac{\theta}{\epsilon}-t\right)\right) \mathrm{e}^{2 \omega_{\theta}+t-\frac{\theta}{\epsilon}} d t \\
-k_{\theta, 1}(0) \int_{\frac{\theta}{\epsilon}-2 \omega_{\theta}}^{\frac{\theta}{\epsilon}}(\dot{\gamma}(\theta)-\dot{\gamma}(\epsilon t)) p_{\epsilon}\left(t, \sigma_{c}-\dot{\gamma}(\theta)\left(\frac{\theta}{\epsilon}-t\right)\right) d t \\
-\int_{0}^{\frac{\theta}{\epsilon}-2 \omega_{\theta}} \int_{\sigma_{c}-\dot{\gamma}(\theta)\left(\frac{\theta}{\epsilon}-t\right)}^{-\sigma_{c}} \frac{\dot{\gamma}(\theta)-\dot{\gamma}(\epsilon t)}{\dot{\gamma}(\theta)} p_{\epsilon}(t, \sigma) \mathrm{e}^{\frac{\sigma+\sigma_{c}}{\dot{\gamma}(\theta)}}\left(k_{\theta, 1}+\dot{k}_{\theta, 1}\right)\left(\frac{\theta}{\epsilon}-t+\frac{\sigma-\sigma_{c}}{\dot{\gamma}(\theta)}\right) d \sigma d t \\
-\int_{\frac{\theta}{\epsilon}-2 \omega_{\theta}}^{\frac{\theta}{\epsilon}} \int_{\sigma_{c}-\dot{\gamma}(\theta)\left(\frac{\theta}{\epsilon}-t\right)}^{-\sigma_{c}} \frac{\dot{\gamma}(\theta)-\dot{\gamma}(\epsilon t)}{\dot{\gamma}(\theta)} p_{\epsilon}(t, \sigma) \dot{k}_{\theta, 1}\left(\frac{\theta}{\epsilon}-t+\frac{\sigma-\sigma_{c}}{\dot{\gamma}(\theta)}\right) d \sigma d t \\
-\int_{0}^{\frac{\theta}{\epsilon}} \int_{-\sigma_{c}}^{\sigma_{c}} \frac{\dot{\gamma}(\theta)-\dot{\gamma}(\epsilon t)}{\dot{\gamma}(\theta)} p_{\epsilon}(t, \sigma) \dot{k}_{\theta, 1}\left(\frac{\theta}{\epsilon}-t+\frac{\sigma-\sigma_{c}}{\dot{\gamma}(\theta)}\right) d \sigma d t
\end{array}
$$

Using Lemma 5.4. $k_{\theta, 1}$ satisfies $\dot{k}_{\theta, 1}(t)+k_{\theta, 1}(t)-k_{\theta, 1}\left(t-\omega_{\theta}\right)=0$ (this is a consequence of (5.11) and (5.16) so that (using (5.17):

$$
\left|\dot{k}_{\theta, 1}(t)\right| \leq C_{0}\left(1+\mathrm{e}^{b \frac{\sigma_{c}}{m_{\dot{\gamma}}}}\right) \mathrm{e}^{-b t}
$$

Using the Lipschitz property of $\dot{\gamma}$, the $L^{\infty}$-bound (4.3) of $p_{\epsilon}$ and estimates (5.17) and 6.29 of $k_{\theta, 1}$ and $\dot{k}_{\theta, 1}$, we obtain

$$
\begin{aligned}
& \left|\int_{0}^{\frac{\theta}{\epsilon}} Q_{\epsilon}(t) k_{\theta, 1}\left(\frac{\theta}{\epsilon}-t\right) d t\right| \\
& \leq C_{0} C_{\infty} L_{\dot{\gamma}} \int_{0}^{\frac{\theta}{\epsilon}}(\theta-\epsilon t) \mathrm{e}^{t-\frac{\theta}{\epsilon}} d t \\
& \quad+C_{0}\left(2+\mathrm{e}^{b \frac{\sigma_{c}}{m_{\dot{\gamma}}}}\right) C_{\infty} L_{\dot{\gamma}} \int_{0}^{\frac{\theta}{\epsilon}}(\theta-\epsilon t) \frac{\mathrm{e}^{b\left(t-\frac{\theta}{\epsilon}\right)}-\mathrm{e}^{t-\frac{\theta}{\epsilon}}}{1-b} d t \\
& \quad+C_{0} C_{\infty} L_{\dot{\gamma}} \int_{0}^{\frac{\theta}{\epsilon}-2 \omega_{\theta}}(\theta-\epsilon t) \mathrm{e}^{2 \omega_{\theta}+t-\frac{\theta}{\epsilon}} d t \\
& \quad+C_{0} C_{\infty} L_{\dot{\gamma}} \int_{\frac{\theta}{\epsilon}-2 \omega_{\theta}}^{\frac{\theta}{\epsilon}}(\theta-\epsilon t) d t \\
& \quad+C_{0}\left(2+\mathrm{e}^{b \frac{\sigma_{c}}{m_{\dot{\gamma}}}}\right) C_{\infty} L_{\dot{\gamma}} \int_{0}^{\frac{\theta}{\epsilon}-2 \omega_{\theta}}(\theta-\epsilon t) \frac{\mathrm{e}^{b\left(t-\frac{\theta}{\epsilon}+2 \omega_{\theta}\right)}-\mathrm{e}^{t-\frac{\theta}{\epsilon}+2 \omega_{\theta}}}{1-b} d t \\
& \quad+C_{0}\left(1+\mathrm{e}^{b \frac{\sigma_{c}}{m_{\dot{\gamma}}}}\right) C_{\infty} L_{\dot{\gamma}} \int_{\frac{\theta}{\epsilon}-2 \omega_{\theta}}^{\frac{\theta}{\epsilon}}(\theta-\epsilon t) \frac{\mathrm{e}^{-2 \omega_{\theta} b}+\mathrm{e}^{b\left(t-\frac{\theta}{\epsilon}\right)}}{b} d t \\
& \quad+C_{0}\left(1+\mathrm{e}^{b \frac{\sigma_{c}}{m_{\dot{\gamma}}}}\right) C_{\infty} L_{\dot{\gamma}} \int_{0}^{\frac{\theta}{\epsilon}}(\theta-\epsilon t) \mathrm{e}^{b\left(t-\frac{\theta}{\epsilon}\right)} \frac{1+\mathrm{e}^{-2 \omega_{\theta} b}}{b} d t .
\end{aligned}
$$


Using the estimate 6.23 or variants and the bounds 6.7 on $\omega_{\theta}$, one concludes

$$
\left|\int_{0}^{\frac{\theta}{\epsilon}} Q_{\epsilon}(t) k_{\theta, 1}\left(\frac{\theta}{\epsilon}-t\right) d t\right| \leq K C_{\infty} C_{0} L_{\dot{\gamma}} \epsilon .
$$

Step 5: Estimate of $g_{\epsilon}\left(\frac{\theta}{\epsilon}\right)$. Using the decomposition 6.15 and estimates 6.24 and 6.30 , we find

$$
\left|g_{\epsilon}\left(\frac{\theta}{\epsilon}\right)\right| \leq K C_{\infty}\left(1+C_{0}\right) L_{\dot{\gamma}} \epsilon
$$

Moreover, the estimate 5.2 established in Theorem 5.1 yields, using the bounds 6.7 on $\omega_{\theta}$

$$
\left|\phi_{\theta}\left(\frac{\theta}{\epsilon}\right)-\int \chi p_{\infty}(\theta, \cdot)\right| \leq \tilde{C}_{3}\left(\mathrm{e}^{-b \frac{\theta}{\epsilon}}+\mathrm{e}^{-\frac{\theta}{\epsilon}}\right)
$$

where $\tilde{C}_{3}>0$ is independent from $\epsilon$ and $\theta$. By the definition 6.9 of $g_{\epsilon}$, we have $f_{\epsilon}-\int \chi p_{\infty}(\theta, \cdot)=g_{\epsilon}+\left(\phi_{\theta}-\int \chi p_{\infty}(\theta, \cdot)\right)$ so that

$$
\begin{aligned}
\left|f_{\epsilon}\left(\frac{\theta}{\epsilon}\right)-\int \chi p_{\infty}(\theta, \cdot)\right| & \leq \tilde{C}_{3}\left(\mathrm{e}^{-b \frac{\theta}{\epsilon}}+\mathrm{e}^{-\frac{\theta}{\epsilon}}\right)+K C_{\infty}\left(1+C_{0}\right) L_{\dot{\gamma} \epsilon} \\
& \leq C_{3}\left(\mathrm{e}^{-b \frac{\theta}{\epsilon}}+\mathrm{e}^{-\frac{\theta}{\epsilon}}+\epsilon\right)
\end{aligned}
$$

where $C_{3}>0$ is independent from $\epsilon$ and $\theta$. This concludes the proof of 6.4).

Step 6: Estimate of $p_{\epsilon}\left(\frac{\theta}{\epsilon}, \sigma\right)-p_{\infty}(\theta, \sigma)$. Recall that the scalars $\theta>0, \epsilon>0$ are fixed and satisfy $\frac{\theta}{\epsilon}>2 \frac{\sigma_{c}}{m_{\dot{\gamma}}}$. For all $\sigma \in \mathbb{R}$, denote

$$
u_{\epsilon, \theta, \sigma}=\gamma^{-1}(\gamma(\theta)-\epsilon \sigma)
$$

where $\gamma(t)=\int_{0}^{t} \dot{\gamma}(s) d s$. The expression (3.4) of $p$, that was established in Theorem 3.1 reads, for almost all $\sigma \in \mathbb{R}$ such that $\sigma \leq \gamma\left(\frac{\theta}{\epsilon}\right)$,

$$
\begin{aligned}
p_{\epsilon}\left(\frac{\theta}{\epsilon}, \sigma\right)= & p_{0}\left(\sigma-\frac{\gamma(\theta)}{\epsilon}\right) \mathrm{e}^{-\int_{0}^{\frac{\theta}{\epsilon}} \chi\left(\sigma-\frac{\gamma(\theta)}{\epsilon}+\frac{\gamma(\epsilon v)}{\epsilon}\right) d v} \\
& +\frac{f_{\epsilon}\left(\frac{u_{\epsilon, \theta, \sigma}}{\epsilon}\right)}{\dot{\gamma}\left(u_{\epsilon, \theta, \sigma}\right)} \times \begin{cases}0 & \text { if } \sigma<0 \\
1 & \text { if } 0<\sigma \leq \sigma_{c} \\
\mathrm{e}^{-\frac{\theta}{\epsilon}+\frac{1}{\epsilon} \gamma^{-1}\left(\gamma(\theta)-\epsilon \sigma+\epsilon \sigma_{c}\right)} & \text { if } \sigma_{c}<\sigma .\end{cases}
\end{aligned}
$$

Note that the condition $\sigma \leq \gamma\left(\frac{\theta}{\epsilon}\right)$ is not restrictive because we are interested in the limit $\epsilon \rightarrow 0$ for a fixed $\sigma$. The rest of the proof depends on the value of $\sigma$.

Let us start with the case $\sigma<0$. We have

$$
\left|p_{\epsilon}\left(\frac{\theta}{\epsilon}, \sigma\right)\right| \leq\left\|p_{0}\right\|_{L^{\infty}} \mathrm{e}^{-\int_{0}^{\frac{\theta}{\epsilon}} \chi\left(\sigma-\frac{\gamma(\theta)}{\epsilon}+\frac{\gamma(\epsilon v)}{\epsilon}\right) d v} .
$$

We now apply Lemma 6.2 with $t=\frac{\theta}{\epsilon}>\frac{2 \sigma_{c}}{m_{\dot{\gamma}}}$ and $\gamma_{\epsilon}(t)=\frac{1}{\epsilon} \gamma(\epsilon t)$ and obtain

$$
\left|p_{\epsilon}\left(\frac{\theta}{\epsilon}, \sigma\right)\right| \leq\left\|p_{0}\right\|_{L^{\infty}} \mathrm{e}^{\frac{2 \sigma_{c}}{m_{\dot{\gamma}}}-\frac{\theta}{\epsilon}} \leq K \mathrm{e}^{-\frac{\theta}{\epsilon}}
$$


Notice that 6.32 actually holds for all $\sigma \in \mathbb{R}$ (this will be used below). From the expression 2.3) of $p_{\infty}, p_{\infty}(\theta, \sigma)=0$ when $\sigma<0$. This gives 6.5 for almost all $\sigma<0$.

Let us now consider the case $\sigma \in\left(0, \sigma_{c}\right]$. Let us introduce the notation $f_{\infty}(\theta)=$ $\int \chi(\sigma) p_{\infty}(\theta, \sigma) d \sigma$. We have, for all positive $\sigma$,

$$
\begin{aligned}
& \left|\frac{f_{\epsilon}\left(\frac{u_{\epsilon, \theta, \sigma}}{\epsilon}\right)}{\dot{\gamma}\left(u_{\epsilon, \theta, \sigma}\right)}-\frac{1}{\sigma_{c}+\dot{\gamma}(\theta)}\right| \\
& \leq \frac{1}{m_{\dot{\gamma}}}\left|f_{\epsilon}\left(\frac{u_{\epsilon, \theta, \sigma}}{\epsilon}\right)-f_{\infty}\left(u_{\epsilon, \theta, \sigma}\right)\right|+\frac{1}{m_{\dot{\gamma}}}\left|f_{\infty}\left(u_{\epsilon, \theta, \sigma}\right)-f_{\infty}(\theta)\right| \\
& \quad+\left|\frac{f_{\infty}(\theta)}{\dot{\gamma}\left(u_{\epsilon, \theta, \sigma}\right)}-\frac{1}{\sigma_{c}+\dot{\gamma}(\theta)}\right| \\
& \leq \\
& \leq K\left(C_{3}+1\right)\left(\mathrm{e}^{-b \frac{\theta}{\epsilon}}+\mathrm{e}^{-\frac{\theta}{\epsilon}}+\epsilon\right),
\end{aligned}
$$

using the estimate (6.4) on $f_{\epsilon}(\dot{\bar{\epsilon}})-f_{\infty}$, the Lipschitz property and the boundedness of $\dot{\gamma}$ and the expression 2.3 which gives $f_{\infty}(\theta)=\frac{\dot{\gamma}(\theta)}{\sigma_{c}+\dot{\gamma}(\theta)}$. Here, we also used the fact that $u_{\epsilon, \theta, \sigma} \geq \theta-\frac{\epsilon \sigma}{m_{\dot{\gamma}}}$, which is a consequence of the Lipschitz property of $\gamma^{-1}$ : $\left|u_{\epsilon, \theta, \sigma}-\theta\right| \leq \frac{\epsilon \sigma}{m_{\dot{\gamma}}}$. For almost all $\sigma \in\left(0, \sigma_{c}\right]$, we deduce (using again 6.32)

$$
\begin{aligned}
& \left|p_{\epsilon}\left(\frac{\theta}{\epsilon}, \sigma\right)-\frac{1}{\sigma_{c}+\dot{\gamma}(\theta)}\right| \\
& \leq\left|p_{0}\left(\sigma-\frac{\gamma(\theta)}{\epsilon}\right) \mathrm{e}^{-\int_{0}^{\frac{\theta}{\epsilon}} \chi\left(\sigma-\frac{\gamma(\theta)}{\epsilon}+\frac{\gamma(\epsilon v)}{\epsilon}\right) d v}\right|+\left|\frac{f_{\epsilon}\left(\frac{u_{\epsilon, \theta, \sigma}}{\epsilon}\right)}{\dot{\gamma}\left(u_{\epsilon, \theta, \sigma}\right)}-\frac{1}{\sigma_{c}+\dot{\gamma}(\theta)}\right| \\
& \leq K \mathrm{e}^{-\frac{\theta}{\epsilon}}+K\left(C_{3}+1\right)\left(\mathrm{e}^{-b \frac{\theta}{\epsilon}}+\mathrm{e}^{-\frac{\theta}{\epsilon}}+\epsilon\right) \text {. }
\end{aligned}
$$

Moreover, we have, from [2.3), $p_{\infty}(\theta, \sigma)=\frac{1}{\sigma_{c}+\dot{\gamma}(\theta)}$ for almost all $\sigma \in\left(0, \sigma_{c}\right]$. This proves 6.5 in this region of $\sigma$.

We now eventually consider the case $\sigma>\sigma_{c}$. Applying the Taylor-Lagrange theorem on $\gamma^{-1}$ at $\gamma(\theta)$, we have (using the fact that $\dot{\gamma}$ is bounded from above)

$$
\left|\frac{\gamma^{-1}\left(\gamma(\theta)-\epsilon\left(\sigma-\sigma_{c}\right)\right)-\theta}{-\epsilon\left(\sigma-\sigma_{c}\right)}-\frac{1}{\dot{\gamma}(\theta)}\right| \leq K \epsilon\left(\sigma-\sigma_{c}\right)
$$

Using that the function $x \mapsto \mathrm{e}^{-x}$ is 1-Lipschitz on $[0, \infty)$, this implies

$$
\left|\mathrm{e}^{-\frac{\theta}{\epsilon}+\frac{1}{\epsilon} \gamma^{-1}\left(\gamma(\theta)-\epsilon \sigma+\epsilon \sigma_{c}\right)}-\mathrm{e}^{-\frac{\sigma-\sigma_{c}}{\dot{\gamma}(\theta)}}\right| \leq K \epsilon\left(\sigma-\sigma_{c}\right)^{2} .
$$

For almost all $\sigma>\sigma_{c}, p_{\infty}$ reads (see 2.3 )

$$
p_{\infty}(\theta, \sigma)=\frac{1}{\sigma_{c}+\dot{\gamma}(\theta)} \mathrm{e}^{\frac{\sigma_{c}-\sigma}{\dot{\gamma}(\theta)}}
$$


and thus, we have (using 6.32) and 6.33)

$$
\begin{aligned}
&\left|p_{\epsilon}\left(\frac{\theta}{\epsilon}, \sigma\right)-p_{\infty}(\theta, \sigma)\right| \\
& \leq \mid p_{0}\left(\sigma-\frac{\gamma(\theta)}{\epsilon}\right) \mathrm{e}^{-\int_{0}^{\frac{\theta}{\epsilon}} \chi\left(\sigma-\frac{\gamma(\theta)}{\epsilon}+\frac{\gamma(\epsilon v)}{\epsilon}\right) d v \mid} \\
& \quad+\left|\frac{f_{\epsilon}\left(\frac{u_{\epsilon, \theta, \sigma}}{\epsilon}\right)}{\dot{\gamma}\left(u_{\epsilon, \theta, \sigma}\right)}-\frac{1}{\sigma_{c}+\dot{\gamma}(\theta)}\right| \mathrm{e}^{-\frac{\theta}{\epsilon}+\frac{1}{\epsilon} \gamma^{-1}\left(\gamma(\theta)-\epsilon \sigma+\epsilon \sigma_{c}\right)} \\
& \quad+\frac{1}{\sigma_{c}+\dot{\gamma}(\theta)}\left|\mathrm{e}^{-\frac{\theta}{\epsilon}+\frac{1}{\epsilon} \gamma^{-1}\left(\gamma(\theta)-\epsilon \sigma+\epsilon \sigma_{c}\right)}-\mathrm{e}^{-\frac{\sigma-\sigma_{c}}{\dot{\gamma}(\theta)}}\right| \\
& \leq K \mathrm{e}^{-\frac{\theta}{\epsilon}}+K\left(C_{3}+1\right)\left(\mathrm{e}^{-b \frac{\theta}{\epsilon}}+\mathrm{e}^{-\frac{\theta}{\epsilon}}+\epsilon\right)+K \epsilon\left(\sigma-\sigma_{c}\right)^{2} .
\end{aligned}
$$

This proves (6.5) and ends the proof.

7. Macroscopic limit. The purpose of this section is to obtain a macroscopic limit for the equation we have been studying.

For $\epsilon>0$ presumably small, we first introduce the notation $\widetilde{u}(\theta)=u\left(\frac{\theta}{\epsilon}\right)$, for any real-valued function $u$ of the real variable $\theta / \epsilon$. The small parameter $\epsilon$ encodes the discrepancy between the typical time of variation of the macroscopic shear rate $\dot{\gamma}(t)$ and the typical mesoscopic time $t$ with which the solution to 6.2 varies. The parameter $\epsilon$ will therefore be the small parameter on which our macroscopic limit is performed. To obtain a macroscopic limit of our equation 6.2 , we will look at a specific macroscopic time, denoted by $\theta$, related to the mesoscopic time $t$ by $\theta=$ $\epsilon t$. Letting $\epsilon$ vanish, we will obtain the corresponding macroscopic behavior of our mesoscopic quantities. In this latter process, we will of course use the results of the previous section, since formally, given a macroscopic time $\theta$, the corresponding mesoscopic time $t=\theta / \epsilon$ is a long time limit.

We begin by formally multiplying 6.2 respectively by $\sigma$ and $\chi(\sigma)$ and integrating in $\sigma$. We next evaluate at the time $\frac{\theta}{\epsilon}$ the two equations obtained. This gives the following system of equations on $\widetilde{\tau}_{\epsilon}$ and $\widetilde{f}_{\epsilon}$, the quantities associated with $p_{\epsilon}$ solution to 6.2 ,

$$
\left\{\begin{array}{l}
\epsilon \frac{d \widetilde{\tau}_{\epsilon}}{d \theta}(\theta)=-\int \chi(\sigma) \sigma \widetilde{p}_{\epsilon}(\theta, \sigma) d \sigma+\dot{\gamma}(\theta) \\
\epsilon \frac{d \widetilde{f}_{\epsilon}}{d \theta}(\theta)=-\widetilde{f}_{\epsilon}(\theta)+\dot{\gamma}(\theta)\left(\widetilde{p}_{\epsilon}\left(\theta, \sigma_{c}\right)-\widetilde{p}_{\epsilon}\left(\theta,-\sigma_{c}\right)\right) .
\end{array}\right.
$$

The difficulty is that this system is not closed in the couple of unknown functions $\left(\widetilde{\tau}_{\epsilon}, \widetilde{f}_{\epsilon}\right)$ since $\widetilde{p}_{\epsilon}$ still appears. We next intend to "eliminate" $\widetilde{p}_{\epsilon}$ from this system, and thereby obtain a system of ordinary differential equations, the solution of which is an approximation, for $\epsilon$ sufficiently small, of $\widetilde{\tau_{\epsilon}}$ and $\widetilde{f_{\epsilon}}$. There are indeed many options to do so. We present two sets of equations which we can derive and that, in a sense made precise below, are equivalent to system (7.1). The precise results are contained in two theorems we now successively state and prove, namely Theorem 7.1 and Theorem 7.2 .

TheOrem 7.1. For $\epsilon>0$, consider $\tau_{\epsilon}^{*}$ the solution to the following differential 
equation in "macroscopic" time $\theta$ :

$$
\epsilon \frac{d \tau_{\epsilon}^{*}}{d \theta}=-\tau_{\epsilon}^{*}+\frac{\sigma_{c}^{2}}{2\left(\sigma_{c}+\dot{\gamma}(\theta)\right)}+\dot{\gamma}(\theta)
$$

supplied with any initial condition $\tau^{*}(0)$ independent of $\epsilon$. Under the assumptions of Theorem 6.1, consider the function $p_{\epsilon}$ solution to (6.2). Then, there exists a constant $C$, independent from $\epsilon$ and $\theta$ provided $\frac{\theta}{\epsilon}>2 \frac{\sigma_{c}}{m_{\dot{\gamma}}}$ (with $m_{\dot{\gamma}}$ the lower bound of $\dot{\gamma}$ in 6.1), such that

$$
\left|\tau_{\epsilon}\left(\frac{\theta}{\epsilon}\right)-\tau_{\epsilon}^{*}(\theta)\right| \leq C\left(\frac{1}{\theta}+1\right) \epsilon
$$

where (in accordance with 1.5) $\tau_{\epsilon}(t)=\int \sigma p_{\epsilon}(t, \sigma) d \sigma$ denotes the stress associated to $p_{\epsilon}$ solution to (6.2).

REMARK 5. The above result holds whatever the initial condition $\tau^{*}(0)$ for the equation 7.2 . Indeed, we are only considering macroscopic times such that $\frac{\theta}{\epsilon}>2 \frac{\sigma_{c}}{m_{\dot{\gamma}}}$ and, in the limit $\epsilon \rightarrow 0$, the boundary layer in time around $\theta=0$ does not affect the result.

Proof. We first give the arguments to derive system (7.1). In the proof of Theorem 5.1. we established equation 6.11, which reads

$$
\dot{f}_{\epsilon}(t)=-f_{\epsilon}(t)+\dot{\gamma}(\epsilon t)\left(p_{\epsilon}\left(t, \sigma_{c}\right)-p_{\epsilon}\left(t,-\sigma_{c}\right)\right) .
$$

Denoting $\rho$ a function of $\mathcal{D}((0, s)), \rho^{n}$ a mollifier on $\mathbb{R}$ and $\chi^{n}=\rho^{n} * \chi$, we used the test function $\eta^{n}(t, \sigma)=\chi^{n}(\sigma) \rho(t)$ in the weak form (6.12) of equation (6.2) and then passed to the limit $n \rightarrow \infty$. We now establish an equation on $\tau_{\epsilon}$ with the same method.

Denote $I: \sigma \mapsto \sigma, I^{n}=\rho^{n} *\left(I \mathbb{1}_{[-n, n]}\right)$ and use $\eta^{n}=(t, \sigma)=I^{n}(\sigma) \rho(t)$ as a test function in 6.12 :

$$
\begin{aligned}
-\int_{0}^{s} \dot{\rho}(t) \int I^{n} p_{\epsilon}(t, \cdot) d t-\int_{0}^{s} \rho(t) \dot{\gamma}(\epsilon t) \int \dot{I}^{n} p_{\epsilon}(t, \cdot) d t & +\int_{0}^{s} \rho(t) \int \chi I^{n} p_{\epsilon}(t, \cdot) d t \\
& =I^{n}(0) \int_{0}^{s} f_{\epsilon}(t) \rho(t) d t .
\end{aligned}
$$

We pass to the limit $n \rightarrow \infty$, using that the function $t \mapsto\left(\sigma \mapsto p_{\epsilon}(t, \sigma)\right)$ belongs to $C\left([0, s], L^{1}\right)$ (see Theorem 3.1), $\int p_{\epsilon}(t, \cdot)=1$, the dominated convergence theorem for the terms in the left-hand side, and that $I^{n}(0) \rightarrow I(0)=0$ for the right-hand side. We obtain, for all $s>0$,

$$
-\int_{0}^{s} \dot{\rho}(t) \tau_{\epsilon}(t) d t-\int_{0}^{s} \rho(t) \dot{\gamma}(\epsilon t) d t+\int_{0}^{s} \rho(t) \int \chi I p_{\epsilon}(t, \cdot) d t=0
$$

so that

$$
\dot{\tau}_{\epsilon}(t)=-\int \chi(\sigma) \sigma p_{\epsilon}(t, \sigma) d \sigma+\dot{\gamma}(\epsilon t)
$$

Changing the variable $t$ in $\frac{\theta}{\epsilon}$ in equations 6.11) and 7.4, we obtain system (7.1).

Now that we have established system (7.1), we rewrite 7.1a in the form

$$
\epsilon \frac{d \widetilde{\tau_{\epsilon}}}{d \theta}=-\widetilde{\tau_{\epsilon}}+\int_{-\sigma_{c}}^{\sigma_{c}} \sigma \widetilde{p_{\epsilon}}(\theta, \sigma) d \sigma+\dot{\gamma}(\theta),
$$


using the definition of $\chi$. Moreover, we use the expression 2.3) of $p_{\infty}$ to rewrite 7.2 as follows

$$
\epsilon \frac{d \tau_{\epsilon}^{*}}{d \theta}=-\tau_{\epsilon}^{*}+\int_{-\sigma_{c}}^{\sigma_{c}} \sigma p_{\infty}(\theta, \sigma) d \sigma+\dot{\gamma}(\theta)
$$

Subtracting the above two equations yields

$$
\frac{d\left(\widetilde{\tau_{\epsilon}}-\tau_{\epsilon}^{*}\right)}{d \theta}+\frac{\widetilde{\tau_{\epsilon}}-\tau_{\epsilon}^{*}}{\epsilon}=\int_{-\sigma_{c}}^{\sigma_{c}} \sigma \frac{\widetilde{p_{\epsilon}}-p_{\infty}}{\epsilon} d \sigma .
$$

Denote $\varsigma=2 \frac{\sigma_{c}}{m_{\dot{\gamma}}}$ so that $\frac{\theta}{\epsilon}>\varsigma$. We next apply the Duhamel formula and find

$$
\left(\widetilde{\tau_{\epsilon}}-\tau_{\epsilon}^{*}\right)(\theta)=\left(\widetilde{\tau_{\epsilon}}-\tau_{\epsilon}^{*}\right)(\varsigma \epsilon) \mathrm{e}^{\varsigma-\frac{\theta}{\epsilon}}+\int_{\varsigma \epsilon}^{\theta} \mathrm{e}^{\frac{u-\theta}{\epsilon}} \int_{-\sigma_{c}}^{\sigma_{c}} \sigma \frac{\widetilde{p_{\epsilon}}-p_{\infty}}{\epsilon} d \sigma d u
$$

Using the estimate (4.7) on $\tau_{\epsilon}$, the bounds (6.1) on $\dot{\gamma}$, we find

$$
\begin{aligned}
\left|\widetilde{\tau}_{\epsilon}-\tau_{\epsilon}^{*}\right|(\varsigma \epsilon) & \leq\left(1+M_{\dot{\gamma} \varsigma}\right) C_{\tau}+\left(\left|\tau_{0}\right| \mathrm{e}^{-\varsigma}+\int_{0}^{\varsigma} \mathrm{e}^{u-\varsigma}\left(\frac{\sigma_{c}^{2}}{2\left(\sigma_{c}+m_{\dot{\gamma}}\right)}+M_{\dot{\gamma}}\right) d u\right) \\
& \leq K
\end{aligned}
$$

where we recall that $K$ denotes a constant that is independent from $\theta$ and $\epsilon$ and whose precise value may change from one occurrence to another. Inserting the above estimate and the estimate 6.5 on $\left|\widetilde{p}_{\epsilon}-p_{\infty}\right|$ in $(7.5)$ yields

$$
\begin{aligned}
\left|\widetilde{\tau_{\epsilon}}-\tau_{\epsilon}^{*}\right|(\theta) & \leq K\left[\mathrm{e}^{\varsigma-\frac{\theta}{\epsilon}}+\int_{0}^{\theta} \mathrm{e}^{\frac{u-\theta}{\epsilon}}\left(\frac{\mathrm{e}^{-b \frac{u}{\epsilon}}}{\epsilon}+\frac{\mathrm{e}^{-\frac{u}{\epsilon}}}{\epsilon}+1\right) d u\right] \\
& \leq K\left(\mathrm{e}^{-\frac{\theta}{\epsilon}}+\frac{1}{1-b}\left(\mathrm{e}^{-b \frac{\theta}{\epsilon}}-\mathrm{e}^{-\frac{\theta}{\epsilon}}\right)+\frac{\theta}{\epsilon} \mathrm{e}^{-\frac{\theta}{\epsilon}}+\epsilon\right) \\
& \leq K\left(\frac{b}{|1-b|} \frac{\mathrm{e}^{-1}}{\theta}+\frac{1}{|1-b|} \frac{\mathrm{e}^{-1}}{b \theta}+\frac{4 \mathrm{e}^{-2}}{\theta}+1\right) \epsilon,
\end{aligned}
$$

using that the functions $x \mapsto x \mathrm{e}^{-x}$ and $x \mapsto x^{2} \mathrm{e}^{-x}$ are respectively bounded by $\mathrm{e}^{-1}$ and $4 \mathrm{e}^{-2}$ on $\mathbb{R}_{+}$in order to derive the last line. This concludes the proof. $\mathrm{\square}$

TheOREM 7.2. For $\epsilon>0$, consider $\left(\tau_{\epsilon}^{* *}, f_{\epsilon}^{* *}\right)$ satisfying the following system of equations in macroscopic time $\theta$ :

$$
\left\{\begin{array}{l}
\epsilon \frac{d \tau_{\epsilon}^{* *}}{d \theta}(\theta)=-\kappa(\theta) f_{\epsilon}^{* *}(\theta) \tau_{\epsilon}^{* *}(\theta)+\dot{\gamma}(\theta), \\
\epsilon \frac{d f_{\epsilon}^{* *}}{d \theta}(\theta)=-f_{\epsilon}^{* *}(\theta)+\frac{\dot{\gamma}(\theta)}{\sigma_{c}+\dot{\gamma}(\theta)},
\end{array}\right.
$$

where we have introduced the notation

$$
\kappa(\theta)=\frac{2}{1+\frac{1}{\left(1+\frac{\sigma_{c}}{\dot{\gamma}(\theta)}\right)^{2}}},
$$

and where the equations are supplied with any couple of scalars (independent of $\epsilon$ ) $\left(\tau^{* *}(0), f^{* *}(0)\right)$ as initial conditions. Consider $\theta>0$ such that $\frac{\theta}{\epsilon}>2 \frac{\sigma_{c}}{m_{\dot{\gamma}}}$. Under the 
assumptions of Theorem 6.1, consider $p_{\epsilon}$ the solution of 6.2. Then, there exists a constant $C$ independent from $\epsilon$ and $\theta$ such that,

$$
\left|\tau_{\epsilon}\left(\frac{\theta}{\epsilon}\right)-\tau_{\epsilon}^{* *}(\theta)\right|+\left|f_{\epsilon}\left(\frac{\theta}{\epsilon}\right)-f_{\epsilon}^{* *}(\theta)\right| \leq C\left(\frac{1}{\theta}+1\right) \epsilon .
$$

REMARK 6. As in Theorem 7.1, the above result holds whatever the set of initial conditions $\left(\tau^{* *}(0), f^{* *}(0)\right)$ for the system of differential equations (7.8).

Proof. The proof falls in three steps. We first study $f_{\epsilon}$, then an auxiliary function $\beta_{\epsilon}$ and finally $\tau_{\epsilon}$.

Step 1: Approximation of $f_{\epsilon}^{* *}$. Applying the Duhamel formula to $7.8 \mathrm{~b}$ yields

$$
\begin{aligned}
f_{\epsilon}^{* *}(\theta) & =f_{0} \mathrm{e}^{-\frac{\theta}{\epsilon}}+\frac{1}{\epsilon} \int_{0}^{\theta} \frac{\dot{\gamma}(v)}{\sigma_{c}+\dot{\gamma}(v)} \mathrm{e}^{\frac{v-\theta}{\epsilon}} d v \\
& =f_{0} \mathrm{e}^{-\frac{\theta}{\epsilon}}+\frac{\dot{\gamma}(\theta)}{\sigma_{c}+\dot{\gamma}(\theta)}-\frac{\dot{\gamma}(0)}{\sigma_{c}+\dot{\gamma}(0)} \mathrm{e}^{-\frac{\theta}{\epsilon}}-\int_{0}^{\theta} \frac{\sigma_{c} \ddot{\gamma}(v)}{\left(\sigma_{c}+\dot{\gamma}(v)\right)^{2}} \mathrm{e}^{\frac{v-\theta}{\epsilon}} d v .
\end{aligned}
$$

Using the Lipschitz property of $\dot{\gamma}$ and denoting by $f_{\infty}(\theta)=\int \chi(\sigma) p_{\infty}(\theta, \sigma) d \sigma=$ $\frac{\dot{\gamma}(\theta)}{\sigma_{c}+\dot{\gamma}(\theta)}$ (following (1.4) and $(2.3)$ ), we easily obtain

$$
\left|f_{\epsilon}^{* *}-f_{\infty}\right|(\theta) \leq K\left(\mathrm{e}^{-\frac{\theta}{\epsilon}}+\epsilon\right) .
$$

Collecting the above equation and the estimate 6.4 established in Theorem 6.1 we obtain

$$
\begin{aligned}
\left|\widetilde{f}_{\epsilon}-f_{\epsilon}^{* *}\right|(\theta) & \leq K\left(\mathrm{e}^{-b \frac{\theta}{\epsilon}}+\mathrm{e}^{-\frac{\theta}{\epsilon}}+\epsilon\right) \\
& \leq K\left(\frac{\mathrm{e}^{-1}}{b \theta}+\frac{\mathrm{e}^{-1}}{\theta}+1\right) \epsilon
\end{aligned}
$$

using that the function $x \mapsto x \mathrm{e}^{-x}$ is bounded by $\mathrm{e}^{-1}$ on $\mathbb{R}_{+}$.

Step 2: Introduction of the auxiliary function $\beta_{\epsilon}$. We now introduce

$$
\beta(t)=\int \chi(\sigma) \sigma p(t, \sigma) d \sigma,
$$

defined for a density probability $p$ such that $\sigma p \in L^{1}$. Denote $\rho$ a function of $\mathcal{D}((0, s))$, $\rho^{n}$ a mollifier on $\mathbb{R}$ and $I: \sigma \mapsto \sigma$. Using $\eta^{n}(t, \sigma)=\left(I \chi \mathbb{1}_{[-n, n]}\right) * \rho^{n}(\sigma) \rho(t)$ as test function in 6.12 and passing to the limit with the same arguments as in (6.13), we obtain

$$
\epsilon \frac{d \widetilde{\beta}_{\epsilon}}{d \theta}(\theta)+\widetilde{\beta}_{\epsilon}(\theta)=\dot{\gamma}(\theta)\left(\widetilde{f}_{\epsilon}(\theta)+\sigma_{c}\left(\widetilde{p}_{\epsilon}\left(\theta, \sigma_{c}\right)+\widetilde{p}_{\epsilon}\left(\theta,-\sigma_{c}\right)\right)\right) .
$$

Consider $\beta_{\epsilon}^{*}$ the solution of the ordinary differential equation

$$
\epsilon \frac{d \beta_{\epsilon}^{*}}{d \theta}+\beta_{\epsilon}^{*}=\dot{\gamma}(\theta)\left(f_{\epsilon}^{* *}+\frac{\sigma_{c}}{\sigma_{c}+\dot{\gamma}(\theta)}\right)
$$

supplied with a scalar $\beta_{0}$ as initial condition. Subtracting the above two equations and using that (see 2.3) $p_{\infty}\left(\theta, \sigma_{c}\right)=\frac{1}{\sigma_{c}+\dot{\gamma}(\theta)}$ and $p_{\infty}\left(\theta,-\sigma_{c}\right)=0$, we obtain

$$
\frac{d\left(\widetilde{\beta}_{\epsilon}-\beta_{\epsilon}^{*}\right)}{d \theta}+\frac{\widetilde{\beta}_{\epsilon}-\beta_{\epsilon}^{*}}{\epsilon}=G_{\epsilon}(\theta)
$$


with

$$
G_{\epsilon}(\theta)=\dot{\gamma}(\theta) \frac{\widetilde{f}_{\epsilon}-f_{\epsilon}^{* *}}{\epsilon}(\theta)+\dot{\gamma}(\theta) \sigma_{c} \frac{\widetilde{p_{\epsilon}}-p_{\infty}}{\epsilon}\left(\theta, \sigma_{c}\right)+\dot{\gamma}(\theta) \sigma_{c} \frac{\widetilde{p_{\epsilon}}-p_{\infty}}{\epsilon}\left(\theta,-\sigma_{c}\right) .
$$

Recall that $\varsigma=2 \frac{\sigma_{c}}{m_{\dot{\gamma}}}$ and $\theta>\varsigma \epsilon$. Applying the Duhamel formula yields,

$$
\left(\widetilde{\beta}_{\epsilon}-\beta_{\epsilon}^{*}\right)(\theta)=\left(\widetilde{\beta}_{\epsilon}-\beta_{\epsilon}^{*}\right)(\varsigma \epsilon) \mathrm{e}^{\varsigma-\frac{\theta}{\epsilon}}+\int_{\varsigma \epsilon}^{\theta} \mathrm{e}^{\frac{u-\theta}{\epsilon}} G_{\epsilon}(u) d u
$$

Using the upper bound (4.7), $\widetilde{\beta}_{\epsilon}$ satisfies

$$
\left|\widetilde{\beta}_{\epsilon}(\varsigma \epsilon)\right| \leq \int|\sigma| \chi p_{\epsilon}(\varsigma, \cdot) \leq \int|\sigma| p_{\epsilon}(\varsigma, \cdot) \leq\left(1+M_{\dot{\gamma} \varsigma}\right) C_{\tau} \leq K .
$$

Moreover, $\beta_{\epsilon}^{*}$ solution of 7.15 satisfies

$$
\begin{aligned}
\left|\beta_{\epsilon}^{*}(\varsigma \epsilon)\right| & \leq\left|\beta_{0}\right| \mathrm{e}^{-\varsigma}+\int_{0}^{\varsigma} \mathrm{e}^{u-\varsigma}\left(\left(\left|f_{0}\right|+\frac{M_{\dot{\gamma}}}{\sigma_{c}+m_{\dot{\gamma}}}\right)+\frac{\sigma_{c}}{\sigma_{c}+m_{\dot{\gamma}}}\right) d u \\
& \leq K .
\end{aligned}
$$

Using (7.13), the boundedness of $\dot{\gamma}$ and the estimate 6.5 on $\widetilde{p}_{\epsilon}-p_{\infty}$, the right-hand side $G_{\epsilon}(\theta)$ of 7.16 satisfies,

$$
G_{\epsilon}(\theta) \leq K\left(\frac{\mathrm{e}^{-b \frac{\theta}{\epsilon}}}{\epsilon}+\frac{\mathrm{e}^{-\frac{\theta}{\epsilon}}}{\epsilon}+1\right) .
$$

Inserting the three above inequalities in 7.17 implies

$$
\left|\widetilde{\beta}_{\epsilon}-\beta_{\epsilon}^{*}\right|(\theta) \leq K\left(\mathrm{e}^{-b \frac{\theta}{\epsilon}}+\mathrm{e}^{-\frac{\theta}{\epsilon}}+\epsilon\right) .
$$

Additionally, applying the Duhamel formula to 7.15 and using the explicit formula $f_{\infty}(\theta)=\frac{\dot{\gamma}(\theta)}{\sigma_{c}+\dot{\gamma}(\theta)}$ yield

$$
\beta_{\epsilon}^{*}(\theta)-\int_{0}^{\theta} \frac{\dot{\gamma}(u)}{\epsilon} \mathrm{e}^{\frac{u-\theta}{\epsilon}} d u=\beta_{0} \mathrm{e}^{-\frac{\theta}{\epsilon}}+\int_{0}^{\theta} \frac{\dot{\gamma}(u)}{\epsilon}\left(f_{\epsilon}^{* *}(u)-f_{\infty}\right) \mathrm{e}^{\frac{u-\theta}{\epsilon}} d u .
$$

Using the Lipschitz property of $\dot{\gamma}$ and the estimate 7.12 , we obtain

$$
\left|\beta_{\epsilon}^{*}-\dot{\gamma}\right|(\theta) \leq K\left(\mathrm{e}^{-\frac{\theta}{\epsilon}}+\epsilon\right) .
$$

Combining (7.18) and (7.19) leads to

$$
\left|\widetilde{\beta_{\epsilon}}-\dot{\gamma}\right|(\theta) \leq K\left(\mathrm{e}^{-b \frac{\theta}{\epsilon}}+\mathrm{e}^{-\frac{\theta}{\epsilon}}+\epsilon\right)
$$

and eventually,

$$
\begin{aligned}
\left|\widetilde{\tau}_{\epsilon}-\tau_{\infty}\right|(\theta) & \leq\left|\int \chi \sigma\left(\widetilde{p_{\epsilon}}-p_{\infty}\right)\right|+\left|\int(1-\chi) \sigma\left(\widetilde{p_{\epsilon}}-p_{\infty}\right) d \sigma\right| \\
& \leq K\left(\mathrm{e}^{-b \frac{\theta}{\epsilon}}+\mathrm{e}^{-\frac{\theta}{\epsilon}}+\epsilon\right),
\end{aligned}
$$

respectively using 7.20 and 6.5 to estimate the two terms of the right-hand side. Here we have used the notation $\tau_{\infty}(\theta)=\int \sigma p_{\infty}(\theta, \sigma) d \sigma$, and the fact that $\int \chi(\sigma) \sigma p_{\infty}(\theta, \sigma) d \sigma=$ $\dot{\gamma}(\theta)$. 
Step 3: Approximation of $\tau_{\epsilon}$. We now turn to $\left(\widetilde{\tau_{\epsilon}}-\tau_{\epsilon}^{* *}\right)$. Combining 7.1a) and $7.8 \mathrm{a}$ yields

$$
\begin{aligned}
\epsilon \frac{d\left(\widetilde{\tau_{\epsilon}}-\tau_{\epsilon}^{* *}\right)}{d \theta}= & +\kappa f_{\epsilon}^{* *} \tau_{\epsilon}^{* *}-\int \chi \sigma \tilde{p}_{\epsilon} \\
= & -\kappa f_{\infty}\left(\widetilde{\tau_{\epsilon}}-\tau_{\epsilon}^{* *}\right)-\kappa \tau_{\epsilon}^{* *}\left(f_{\infty}-f_{\epsilon}^{* *}\right) \\
& -\kappa f_{\infty}\left(\tau_{\infty}-\widetilde{\tau_{\epsilon}}\right)-\left(-\kappa f_{\infty} \tau_{\infty}+\int \chi \sigma p_{\infty}\right)-\int \chi \sigma\left(\widetilde{p_{\epsilon}}-p_{\infty}\right) .
\end{aligned}
$$

From the formula 2.3 on $p_{\infty}$, we compute $\int \chi \sigma p_{\infty}(\theta, \cdot)=\dot{\gamma}(\theta), f_{\infty}(\theta)=\frac{\dot{\gamma}(\theta)}{\sigma_{c}+\dot{\gamma}(\theta)}$, $\tau_{\infty}(\theta)=\frac{1}{2}\left(\frac{\dot{\gamma}(\theta)^{2}}{\sigma_{c}+\dot{\gamma}(\theta)}+\sigma_{c}+\dot{\gamma}(\theta)\right)$ so that the term $-\kappa f_{\infty} \tau_{\infty}+\int \chi \sigma p_{\infty}$ cancels out because of the definition 7.9 of $\kappa$. We therefore obtain

$$
\frac{d\left(\widetilde{\tau_{\epsilon}}-\tau_{\epsilon}^{* *}\right)}{d \theta}+\frac{\kappa f_{\infty}}{\epsilon}\left(\widetilde{\tau_{\epsilon}}-\tau_{\epsilon}^{* *}\right)=H_{\epsilon}(\theta)
$$

with

$$
H_{\epsilon}(\theta)=-\kappa \tau_{\epsilon}^{* *} \frac{f_{\infty}-f_{\epsilon}^{* *}}{\epsilon}(\theta)-\kappa f_{\infty} \frac{\tau_{\infty}-\widetilde{\tau_{\epsilon}}}{\epsilon}(\theta)-\int \chi(\sigma) \sigma \frac{\widetilde{p_{\epsilon}}-p_{\infty}}{\epsilon}(\theta, \sigma) d \sigma .
$$

We have $\kappa \geq 1$ (see 7.9 ) ) so that

$$
\kappa f_{\infty} \geq \frac{1}{1+\frac{\sigma_{c}}{m_{\dot{\gamma}}}} .
$$

The Duhamel formula then implies

$$
\left|\widetilde{\tau_{\epsilon}}-\tau_{\epsilon}^{* *}\right|(\theta) \leq\left|\widetilde{\tau_{\epsilon}}-\tau_{\epsilon}^{* *}\right|(\varsigma \epsilon) \mathrm{e}^{\frac{1}{1+\frac{\sigma_{C}}{m_{\dot{\gamma}}}}\left(\varsigma-\frac{\theta}{\epsilon}\right)}+\int_{\varsigma \epsilon}^{\theta} \mathrm{e}^{\frac{1}{1+\frac{\sigma_{c}}{m_{\dot{\gamma}}}} \frac{u-\theta}{\epsilon}}\left|H_{\epsilon}(u)\right| d u
$$

Using the upper bound (4.7), $\widetilde{\tau}_{\epsilon}$ satisfies

$$
\left|\widetilde{\tau}_{\epsilon}(\varsigma \epsilon)\right| \leq\left(1+M_{\dot{\gamma} \varsigma}\right) C_{\tau} \leq K .
$$

Moreover, the solution $\tau_{\epsilon}^{* *}$ of 7.8 satisfies (using the non negativity of $\kappa$ and $f_{\epsilon}^{* *}$ ):

$$
\begin{aligned}
\tau_{\epsilon}^{* *}(\theta) & =\mathrm{e}^{-\frac{1}{\epsilon} \int_{0}^{\theta} \kappa(s) f_{\epsilon}^{* *}(s) d s} \tau^{* *}(0)+\frac{1}{\epsilon} \int_{0}^{\theta} \dot{\gamma}(s) \mathrm{e}^{-\frac{1}{\epsilon} \int_{s}^{\theta} \kappa(r) f_{\epsilon}^{* *}(r) d r} d s \\
& \leq \tau^{* *}(0)+M_{\dot{\gamma}} \frac{\theta}{\epsilon}
\end{aligned}
$$

so that

$$
\left|\tau_{\epsilon}^{* *}(\varsigma \epsilon)\right| \leq K(1+\varsigma) .
$$

Collecting (6.4) established in Theorem 6.1, 7.20 and 7.21), the right-hand side $H_{\epsilon}(u)$ of 7.22 satisfies

$$
H_{\epsilon}(u) \leq K\left(\frac{\mathrm{e}^{-b \frac{u}{\epsilon}}}{\epsilon}+\frac{\mathrm{e}^{-\frac{u}{\epsilon}}}{\epsilon}+1\right)
$$


Inserting the three above inequalities in 7.24 implies

$$
\begin{aligned}
& \left|\widetilde{\tau_{\epsilon}}-\tau_{\epsilon}^{* *}\right|(\theta) \leq K\left(\mathrm{e}^{-\frac{1}{1+\frac{\sigma_{c}}{m_{\dot{\gamma}}} \frac{\theta}{\epsilon}}}+\int_{0}^{\theta} \mathrm{e}^{\frac{1}{1+\frac{\sigma_{c}}{m_{\dot{\gamma}}}} \frac{u-\theta}{\epsilon}}\left(\frac{\mathrm{e}^{-b \frac{u}{\epsilon}}}{\epsilon}+\frac{\mathrm{e}^{-\frac{u}{\epsilon}}}{\epsilon}+1\right) d u\right) \\
& \leq K\left(\frac{1}{\theta}+1\right) \epsilon .
\end{aligned}
$$

We end this section with a discussion on the two macroscopic limits 7.2 and 77.8 we have obtained. First, as mentioned above, there are many ways to close the system (7.1) in the limit $\epsilon \rightarrow 0$. We have proposed here two possible macroscopic limits, which are indeed close up to terms of order $O(\epsilon)$ to the original problem (6.2).

Second, we would like to argue that the system (7.8) derived in Theorem 7.2 is physically more relevant. Indeed, up to changing the coefficient $\kappa(\theta)$ defined by $(7.9)$ by a constant, this system belongs to a class of equations introduced in [7, 12, to model the evolution of aging fluids. These equations read (see [12, Eq. (1)])

$$
\left\{\begin{array}{l}
\frac{\partial \tau}{\partial t}=-f \tau+\dot{\gamma} \\
\frac{\partial f}{\partial t}=-U(f)+V(f, \tau, \dot{\gamma}),
\end{array}\right.
$$

where $U$ and $V$ are positive functions. The formal similarity between 7.8 and 7.25 is clear.

For this class of systems, the fluidity $f$ appears as the inverse of the relaxation time for the stress $\tau$ in equation $7.25 \mathrm{a}$. In equation $7.25 \mathrm{~b}$ the evolution results from the competition between the two terms with opposite signs. Aging, meaning solidification of the fluid, is modeled by the negative term. It makes the fluidity decrease so that the relaxation phenomenon is slower with time. The opposite effect, flow-induced rejuvenation, is modeled by the positive term, which makes the fluidity (the inverse relaxation time) increase.

Note that the assumption $\kappa(\theta)$ constant is a reasonable approximation when $\dot{\gamma}$ is small. In this case, system 77.8 is close to system 8.2 , which is indeed of the form 7.25 . In section 8 , we present numerical results that confirm that the solutions to 7.8$)$ and 8.2 are indeed close when $\dot{\gamma}$ is small.

8. Numerical experiments. This section is devoted to some numerical experiments. We consider three different situations, depending on the value of the function $\dot{\gamma}(t)$ for $t \in[0, T]$.

(i) In our first series of tests, we consider the constant shear rate $\dot{\gamma}(t) \equiv \dot{\gamma}_{\infty}$, for different values $0.1,0.2, \cdots, 0.8$ of $\dot{\gamma}_{\infty}$. In that case, the final time is $T=40$

(ii) In our second series of tests, we consider $\dot{\gamma}(t)=t$, and the final time $T=\frac{\theta}{\epsilon}$ with $\theta=1$ and $\epsilon$ varying between the values 0.005 and 0.05 .

(iii) In our third and final series of tests, we take $\dot{\gamma}(t)=0.01 \cdot t$, and the same values of $T, \theta$, and $\epsilon$ as in case (ii).

In all our tests, the reference equation, namely (1.1) (or more precisely (6.2)), is simulated over the time interval $[0, T]$. Since in theory it is posed on the whole real line, we need to truncate the domain and thus actually solve the equation on the bounded interval $\sigma \in\left[-M_{\sigma}, M_{\sigma}\right]$ (with periodic boundary conditions), for $M_{\sigma}=$ 10 , with a constant space step $\Delta \sigma=\frac{M_{\sigma}}{2.10^{5}}$. The initial condition $p_{0}$ is the normal 
centered Gaussian density, appropriately renormalized on the interval $\left[-M_{\sigma}, M_{\sigma}\right]$. The threshold value for the stress is $\sigma_{c}=2$. The time discretization is performed using a splitting method: over the time interval $[n \Delta t,(n+1) \Delta t]$,

$$
\left\{\begin{aligned}
\frac{\partial p^{n+\frac{1}{2}}}{\partial t} & =-\chi p^{n+\frac{1}{2}}+\left(\int \chi p^{n+\frac{1}{2}}\right) \delta_{0} \\
\frac{\partial p^{n+1}}{\partial t}+\dot{\gamma}(\epsilon n \Delta t) \frac{\partial p^{n+\frac{1}{2}}}{\partial \sigma} & =0 .
\end{aligned}\right.
$$

Equation 8.1a is solved explicitly, pointwise for each $\sigma \neq 0$, while the equation for $\sigma=0$ is indeed solved using the conservation of the total mass of the density $p$. In short, the value of the density at zero is adjusted so that the integral of $p$ is one. See 9 ] for more details. The advection equation $8.1 \mathrm{~b}$ is solved using an upwind finite difference scheme. All computations are performed using $\mathrm{C}++$.

(i) For our first series of tests, performed for the shear rate $\dot{\gamma}(t)=\dot{\gamma}_{\infty}$, we first check the optimality of the long time convergence result stated in Theorem 5.1. For the various values of $\dot{\gamma}_{\infty}$ indicated above, we simulate (1.1). Using a least-square fit, we then estimate, in function of $\dot{\gamma}_{\infty}$, the exponent of the exponential rate of convergence of $\left(\int\left(p(t, \cdot)-p_{\infty}\right)^{2}\right)^{1 / 2}$ to zero as time goes to infinity, more precisely what corresponds to the parameter $b$ of the right-hand side of (5.1). The function $p_{\infty}$ is of course the stationary solution 2.3 , itself function of $\dot{\gamma}_{\infty}$, explicitly determined in our theoretical study. Note that for this practical experiment we intentionally make a confusion between the rate of convergence of the pointwise difference $p(t, \cdot)-p_{\infty}$ in 5.1) and its $L^{2}$ norm. Figure 8.1 shows, as a function of $\dot{\gamma}_{\infty}$, a comparison between the rate of convergence fitted on the numerical results and the theoretical value of the inferior bound on this rate of convergence provided by our theoretical estimate (5.3). The two sets of data agree, thereby showing the quality of our estimate (5.3).

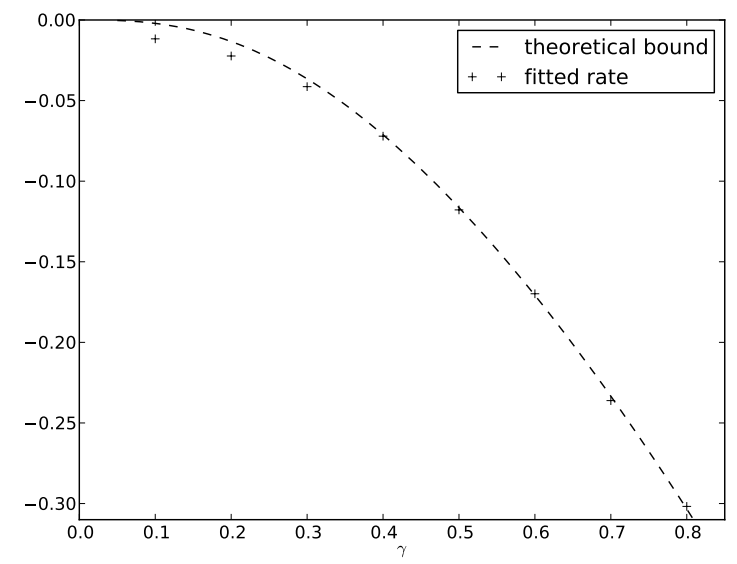

FIG. 8.1. Exponential convergence rate function of $\dot{\gamma}(t)=\dot{\gamma}_{\infty}$

(ii) We next consider $\dot{\gamma}(t)=t$, and implement the change of time scale by taking $\dot{\gamma}(\epsilon t)$ as input for equation (1.1), that is, we solve equation (6.2). We do this for various 
values of the small parameter $\epsilon$. In this case (ii), our purpose is twofold. First, we consider the quantities

$$
\left|\widetilde{f}_{\epsilon}-f_{\infty}\right|(\theta)=\left|\int \chi p_{\epsilon}\left(\frac{\theta}{\epsilon}, \cdot\right)-\int \chi p_{\infty}(\theta, \cdot)\right|
$$

and

$$
\left|\widetilde{\tau_{\epsilon}}-\tau_{\infty}\right|(\theta)=\left|\int \sigma p_{\epsilon}\left(\frac{\theta}{\epsilon}, \cdot\right)-\int \sigma p_{\infty}(\theta, \cdot)\right|
$$

where $p_{\epsilon}$ and $p_{\infty}$ are the solutions to 6.2 and 6.3), respectively. We wish to check that, as the estimate proved in Theorem 6.1 suggests, these two quantities behave linearly in function of $\epsilon$ for small $\epsilon$. The figures 8.2 and 8.3 show this is indeed the case.

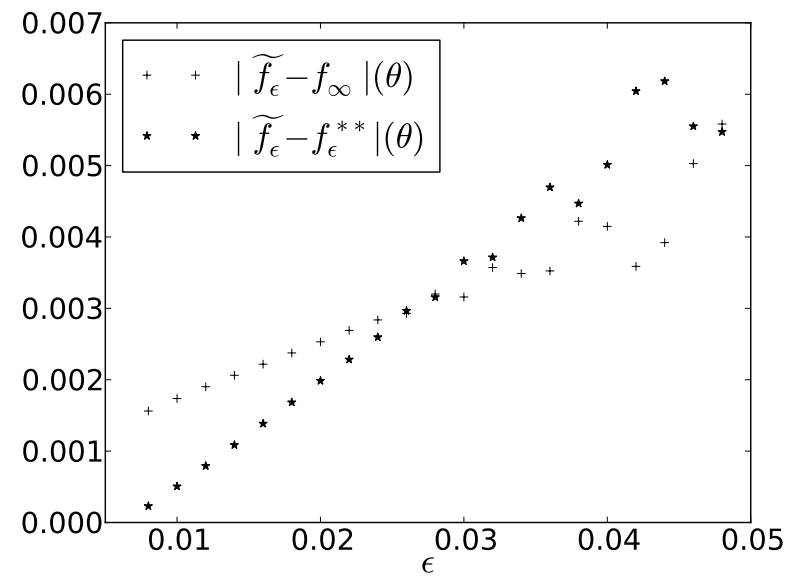

FIG. 8.2. Convergence of $f$

Our second purpose is to illustrate that the macroscopic equations obtained in Theorems 7.1 and 7.2 indeed behave similarly to the original mesoscopic equation (6.2), that is, provide a fluidity $f$ and a stress $\tau$ comparable to that computed from the solution to the latter equation. For the different values of $\epsilon$ mentioned above, we therefore simulate equations 77.2 and 77.8 on the time interval $[0, \theta]$, with the time step $\Delta t=\frac{T}{1.10^{4}}$. The values of $\left|\widetilde{\tau_{\epsilon}}-\tau_{\epsilon}^{*}\right|(\theta),\left|\tilde{f}_{\epsilon}-f_{\epsilon}^{* *}\right|(\theta)$ et $\left|\widetilde{\tau_{\epsilon}}-\tau_{\epsilon}^{* *}\right|(\theta)$ are displayed on Figures 8.2 and 8.3 . We observe that the convergence is linear in $\epsilon$, as predicted by our theoretical results Theorems 7.1 and 7.2. The macroscopic behavior is thus suitably reproduced, up to an error of size $O(\epsilon)$.

(iii) Our final test case addresses the case $\dot{\gamma}(t)=0.01 \cdot t$. We again rescale the time and consider $\dot{\gamma}(\epsilon t)$. A similar experiment as that performed in the previous case (ii) again shows that equation (7.8) reproduces well the stress tensor computed from the solution to equation $(6.2)$, for the different values of $\epsilon$. Simulating $(6.2)$ and $(7.8)$, we compute $\left|\widetilde{\tau_{\epsilon}}-\tau_{\epsilon}^{* *}\right|(\theta)$. The results are displayed on Figure 8.4

But our purpose here is also to illustrate another fact. When $\dot{\gamma}$ is small, and it is indeed the case for our specific choice of $\dot{\gamma}$ in this case (iii), the value of the 


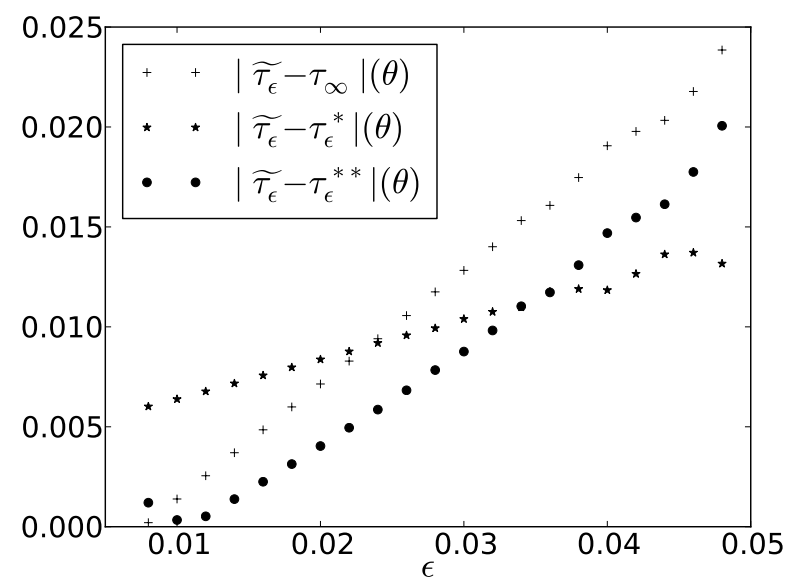

FIG. 8.3. Convergence of $\tau$

parameter $\kappa(\theta)$ defined by $(7.9)$ and appearing in the macroscopic system 7.8 is approximately 2 . System 7.8 is thus close to the system

$$
\left\{\begin{array}{l}
\epsilon \frac{d \tau_{\epsilon}^{* * *}}{d \theta}=-2 f_{\epsilon}^{* * *} \tau_{\epsilon}^{* * *}+\dot{\gamma}(\theta) \\
\epsilon \frac{d f_{\epsilon}^{* * *}}{d \theta}=-f_{\epsilon}^{* * *}+\frac{\dot{\gamma}(\theta)}{\sigma_{c}+\dot{\gamma}(\theta)} .
\end{array}\right.
$$

As explained above, this system of differential equations belongs to the class of systems (7.25) explicitly suggested in [7, 12, as a macroscopic system of evolution of $f$ and $\tau$ for a non Newtonian aging fluid. Our theoretical results of the previous sections can therefore be interpreted as a derivation, from a model at a finer scale, of the macroscopic system (8.2), present in the applicative literature. On Figure 8.4. we indeed check that the stresses solution to the systems 7.8 and 8.2 are close, up to an error of size $O(\epsilon)$, to the stress provided by 6.2 ,

Acknowledgments: We thank the two anonymous referees for their many constructive remarks, and in particular for the alternate proof outlined in Remark 4 .

\section{REFERENCES}

[1] J.-B. Bardet, A. Christen, A. Guillin, F. Malrieu, and P.-A. Zitt, Total variation estimates for the TCP process, Electron. J. Probab., 18 (2013), pp. no. 10, 21.

[2] R. Bellman and K. L. Cooke, Differential-difference equations, Academic Press, New York, 1963.

[3] D. Benoit, Various theoretical and numerical issues related to the simulation of nonNewtonian fluids, PhD thesis, Université Paris Est, 2014, http://tel.archives-ouvertes. fr/tel-00973407/en

[4] L. Bocquet, A. Colin, And A. Ajdari, Kinetic theory of plastic flow in soft glassy materials, Phys. Rev. Lett., 103 (2009), p. 036001.

[5] E. Cancès, I. Catto, AND Y. GATi, Mathematical analysis of a nonlinear parabolic equation arising in the modelling of non-Newtonian flows, SIAM Journal on Mathematical Analysis, 37 (2005), pp. 60-82.

[6] E. CAnCÈs And C. LE BRIS, Convergence to equilibrium of a multiscale model for suspensions, Discrete Contin. Dyn. Syst. Ser. B, 6 (2006), p. 449-470. 


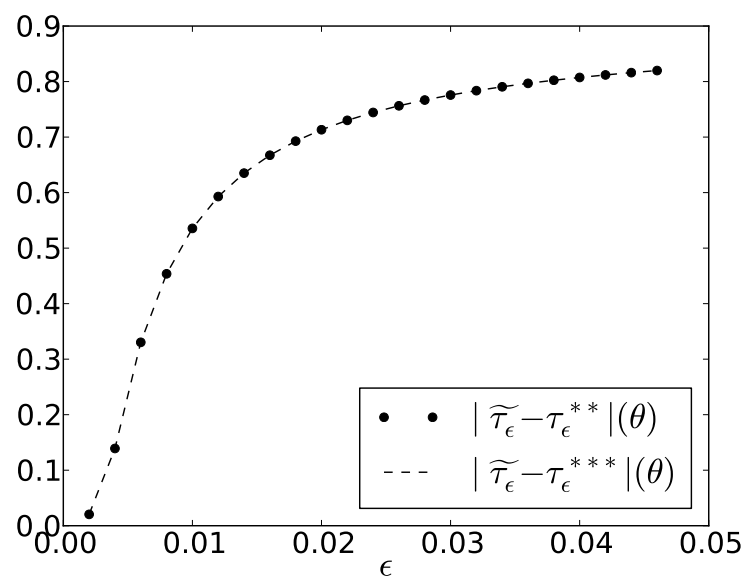

FIG. 8.4. Convergence of $\tau$ in the case $\dot{\gamma}$ small

[7] C. Derec, A. Ajdari, and F. Lequeux, Rheology and aging: a simple approach, Eur. Phys. J. E, 4 (2001), pp. 355-361.

[8] E. Cancès, I. Catto, Y. Gati and C. Le Bris, Well-posedness of a multiscale model for concentrated suspensions SIAM Multiscale Modeling and Simulation, vol. 4, No. 4, pp 10411058, 2005.

[9] Y. Gati, Numerical simulation of a micro-macro model of concentrated suspensions, Int. J. Numer. Meth. Fluids, (2005), pp. 1019-1025

[10] J.K. Hale and S.M.V. Lunel, Introduction to Functional Differential Equations, no. vol. 99 in Applied Mathematical Sciences, Springer, 1993.

[11] P. Hébraud and F. Lequeux, Mode-coupling theory for the pasty rheology of soft glassy materials, Phys. Rev. Lett., 81 (1998), pp. 2934-2937.

[12] G. Picard, A. Ajdari, L. Bocquet, and F. Lequeux, Simple model for heterogeneous flows of yield stress fluids, Phys. Rev. E, 66 (2002), p. 051501. 